
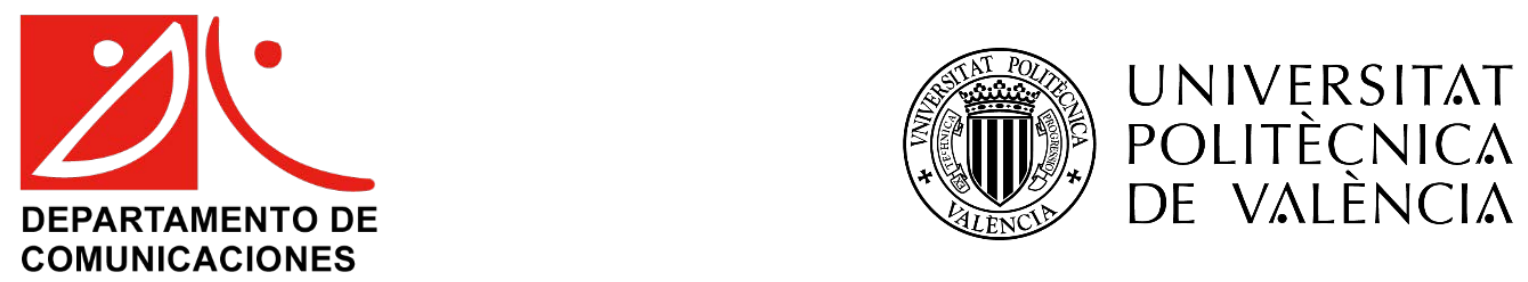

Universitat Politècnica de València

Departamento de Comunicaciones

\title{
OPTICAL PROCESSING BASED ON BRILLOUIN SCATTERING
}

\section{Diego Samaniego Riera}

\author{
Supervisor: Dr. Borja Vidal
}

Thesis submitted to the Universitat Politècnica de València in partial fulfillment of the requirements for the degree of Doctor by Universitat Politècnica de València.

Tesis presentada para la obtención del grado de Doctor por la Universitat Politècnica de València. 

A quien me enseñó a amar, a mi modelo a seguir y a quien cada día me vuelve un poco más loco. Mi madre, mi padre y mi hijo. 



\section{Acknowledgments}

Agradezco a mi tutor y director de tesis, Borja. Quien en todos estos años ha sabido apoyarme, incentivarme y guiarme. Adicionalmente, agradezco a todas las personas que han colaborado en el desarrollo de este sueño. Sin embargo, detallar a cada uno, se convierte en una tarea muy difícil, sobre todo por el temor de olvidar nombrar a alguien.

Desde el primer día que llegué al NTC me sentí como en casa, a pesar de estar a más de 10000 kilómetros de distancia de mi país. Mentiría si digiera que siempre me sentí a gusto, pero mentiría más si no admitiera que la gente con la que he trabajado, todos estos años, me ha hecho sentir que España es mi segundo hogar. Por este motivo quiero agradecer a todos y cada una de las personas que he conocido en el NTC, desde aquellas personas con las que me encontraba en el ascensor y nunca supe su nombre, hasta los más amigos, aquellos compañeros con los que compartíamos grandes charlas en la sala diáfana, o nos veíamos en el almuerzo, en la comida ya sea en el frio invierno o en el caluroso verano, donde una buena cerveza siempre amenizaba las reuniones. Gracias chicos por haberme hecho sentir como en casa.

También quiero agradecer al gobierno del Ecuador por financiar la beca de doctorado que me permitió desarrollar esta investigación. Finalmente, pero no menos importante, quiero agradecer a mi familia "local”, Cris -mi esposa-, Franklin -mi hermano- y Gloria -mi cuñada-. 


\section{Abstract}

Nonlinear effects are valuable tools in the field of optical processing. This Thesis is aimed at contributing with new architectures and methods to this field, in particular to the light-by-light control of polarization and optical filtering of microwave signals.

The manipulation of the polarization properties of light in guided media is crucial in many classical and quantum optical systems. However, the capability of current technology to finely define the state of polarization of particular wavelengths is far from the level of maturity in amplitude control. In Chapter 3, a new approach for all-optical control of the state of polarization with wavelength selectivity based on the change of the phase retardance by means of stimulated Brillouin scattering is presented. Experiments show that any point on the Poincaré sphere can be reached from an arbitrary input state of polarization with little variation of the signal amplitude $(<2.5 \mathrm{~dB})$. Unlike other Brillouin processing schemes, the degradation of the noise figure is small $(1.5 \mathrm{~dB}$ for a full $2 \pi$ rotation). This all-optical polarization controller can forge the development of new polarization-based techniques in optical communication, laser engineering, sensing, quantum systems and light-based probing of chemical and biological systems.

The second area of interest of the Thesis is photonic microwave filtering. Photonics provides an alternative implementation of microwave filters. The features provided by Brillouin scattering are very attractive to design filters with competitive specifications. Chapter 4 is devoted to new schemes for photonic microwave filtering based on SBS. In particular, a method to enhance the filter slope of Brillouin-based photonic microwave filters is presented. This improvement is achieved by the combination of Brillouin gain and loss responses over phase modulated signals. The experimental results show passband responses exhibiting a slope of $16.7 \mathrm{~dB}$ per octave, which corresponds with a 3fold improvement in comparison to the natural Lorentzian response for the same gain. However, the need of three pump waves, i.e. three microwave oscillators, increases the system complexity and make tunability more difficult. To overcome these limitations, a second technique to enhance the slope of a photonic microwave filter based on stimulated Brillouin scattering is proposed, that maintains easy tunability. It relies on exploiting the polarization dependence of Brillouin gain in birefringent fibers. The presence of two orthogonal Brillouin gains/loss in birefringent fibers results in two filter responses 
that can be subtracted in a balanced photodetector to remove the slow Lorentzian decay of the natural Brillouin gain response. Experimental results show that a filter slope of 8.3 $\mathrm{dB} /$ oct can be obtained.

Finally, the Thesis document provides conclusions and future activities opened by this PhD work. 


\section{Resumen}

Los efectos no lineales son herramientas valiosas en el procesamiento óptico. El objetivo de esta Tesis es contribuir con las nuevas arquitecturas y métodos a este campo, en particular al control de la polarización de la luz con luz y filtrado óptico de señales de microondas.

La manipulación de las propiedades de la polarización de la luz en medios guiados es crucial en muchos sistemas ópticos clásicos y cuánticos. Sin embargo, la capacidad de la tecnología actual para definir con precisión el estado de polarización de determinadas longitudes de onda está lejos del nivel de madurez conseguido en el control de la amplitud. En el capítulo 3, se presenta un nuevo enfoque para el control totalmente óptico del estado de polarización con selectividad en longitud de onda, basado en el cambio del retardo fase por medio del stimulated Brillouin scattering. Los experimentos muestran que se puede llegar a cualquier punto de la esfera de Poincaré desde un estado de polarización de entrada arbitrario con tan solo una ligera variación en la amplitud de la señal $(<2.5 \mathrm{~dB})$. A diferencia de otros esquemas de procesamiento Brillouin, la degradación de la figura de ruido es pequeña (1.5 dB para una rotación completa en la esfera, $2 \pi$ ). Este controlador de polarización completamente óptico puede forjar el desarrollo de nuevas técnicas basadas en la polarización en comunicaciones ópticas, ingeniería laser, detección, sistemas cuánticos y sondeo basado en luz de sistemas químicos y biológicos.

La segunda área de interés de la tesis se centra en el filtrado fotónico de microondas. La fotónica proporciona una implementación alternativa a los filtros de microondas. Las características proporcionadas por el scattering de Brillouin son muy atractivas para el diseño de filtro con especificaciones competitivas. El capítulo 4 está dedicado a los nuevos esquemas para el filtrado fotónico de microondas basado en SBS. En particular, se presenta un método para mejorar la pendiente de los filtros fotónicos de microondas basados en Brilouin. Esta mejora se logra mediante la combinación de las respuestas en ganancia y atenuación del Brillouin sobre la señal modulada en fase. Los resultados experimentales muestran una respuesta paso banda que exhibe una pendiente de $16.7 \mathrm{~dB}$ por octava, lo que corresponde con una mejora de 3 veces en comparación con la respuesta Lorentziana natural de la ganancia Brillouin. Sin embargo, la necesidad de 3 ondas de bombeo, es decir tres osciladores de microondas, incrementan la complejidad del 
sistema y dificulta la capacidad de ajuste. Para superar estas limitaciones, se propone una segunda técnica para mejorar la pendiente de un filtro fotónico de microondas basado en scattering de Brillouin estimulado, el cual mantiene una fácil sintonización. Esta propuesta se basa en la dependencia de la polarización de la ganancia del Brillouin en fibras birrefringentes. La presencia de dos respuestas ortogonales de ganancia/atenuación Brillouin en fibras birrefringentes da como resultado dos respuestas del filtro, que pueden ser sustraídas en un fotodetector balanceado para eliminar el lento decaimiento de la respuesta de ganancia natural Lorentziana del Brillouin. Los resultados experimentales muestran que se puede obtener una pendiente del filtro de $8.3 \mathrm{~dB} / \mathrm{oct}$.

Finalmente, el documento de tesis proporciona conclusiones y actividades futuras abiertas por este trabajo de doctorado. 


\section{Resum}

Els efectes no lineals son ferramentes valuoses en el processament òptic. L'objectiu d'aquesta tesi es contribuir amb les noves arquitectures i mètodes a aquest camp, en particular al control de la polarització de la llum amb llum i filtrar òptic de senyals de microones.

La manipulació de les propietats de la polarització de la llum en mitjans guiats es crucial en molts sistemes òptics clàssics i quàntics. No obstant això, la capacitat de la tecnologia actual per definir amb precisió l'estat de polarització de determinades longituds d'ona està lluny del nivell de maduresa aconseguit en el control de l'amplitud. En el capítol 3, es presenta un nou enfocament per al control totalment òptic de l'estat de polarització amb selectivitat en longitud d'ona, basat en el canvi del retard de fase mitjançant el stimulated Brillouin scattering. Els experiments mostren que es pot arribar a qualsevol punt de l'esfera de Poincaré des d'un estat de polarització d'entrada arbitrari amb tant sols una lleugera variació de l'amplitud de la senyal $(<2.5 \mathrm{~dB})$. A diferencia d'altres esquemes de processament Brillouin, la degradació de la figura de soroll es petita (1.5 dB per a una rotació completa en l'esfera, $2 \pi$ ). Aquest controlador de polarització completament òptic pot forjar el desenvolupament de noves tècniques basades en la polarització en comunicacions òptiques, enginyeria làser, detecció, sistemes quàntics y sondeig basat en llum de sistemes químics i biològics.

La segona àrea d'interès de la tesi es centra en el filtrar fotònic de microones. La fotònica proporciona una implementació alternativa als filtres de microones. Les característiques proporcionades per el scattering de Brillouin son molt atractives per al disseny de filtres amb especificacions competitives. El capítol 4 està dedicat als nous esquemes per al filtrat fotònic de microones basat en SBS. En particular, es presenta un mètode per a millorar la pendent dels filtres fotònics de microones basats en Brillouin. Aquesta millora s'aconsegueix mitjançant la combinació de les respostes en guany i atenuació del Brillouin sobre la senyal modulada en fase. Els resultats experimentals mostren una resposta pas banda que exhibeix una pendent de $16.7 \mathrm{~dB}$ per octava, el que correspon amb una millora de 3 vegades en comparació amb la resposta Lorentziana natural del guany Brillouin. Tot i això, la necessitat de 3 ones de bombeig, es a dir tres oscil-ladors de microones, incrementen la complexitat del sistema i dificulta la capacitat d'ajust. Per superar 
aquestes limitacions, es proposa una segona tècnica per millorar la pendent d'un filtre fotònic de microones basat en scattering de Brillouin estimulat, el qual manté una fàcil sintonització. Aquesta proposta es basa en la dependència de la polarització del guany del Brillouin en fibres birefringents. La presència de dos respostes ortogonals de guany/atenuació Brillouin en fibres birefringents dona com a resultat dos respostes del filtre, que poden ser sostretes en un fotodetector balancejat per eliminar el lent decaïment de la resposta de guany natural Lorentziana del Brillouin. Els resultats experimentals mostren que es pot obtenir una pendent del filtre de $8.3 \mathrm{~dB} /$ oct.

Finalment, el document de tesi proporciona conclusions i activitat futures obertes per aquest treball de doctorat. 


\section{Table of contents}

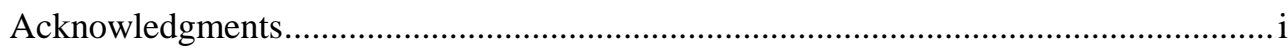

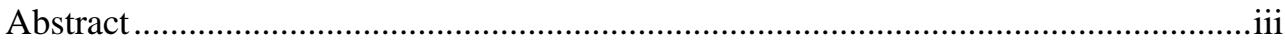

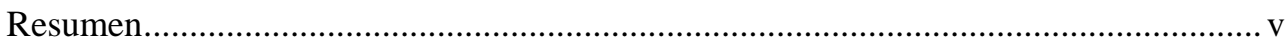

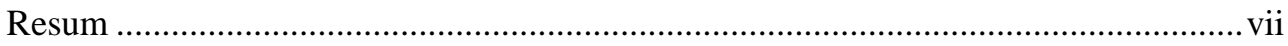

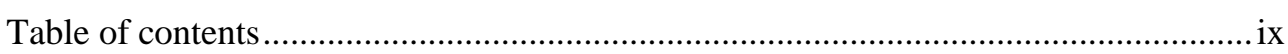

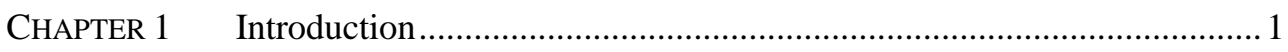

CHAPTER 2 Fundamentals of stimulated Brillouin scattering .................................... 3

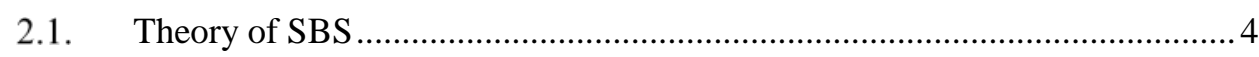

2.1.1. Brillouin Threshold ......................................................................... 9

2.1.2. Polarization Effects on SBS .................................................................. 10

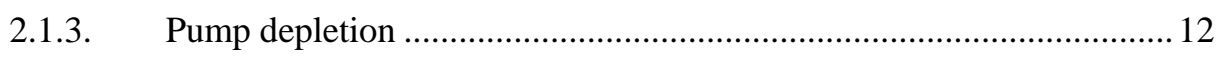

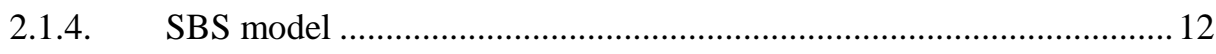

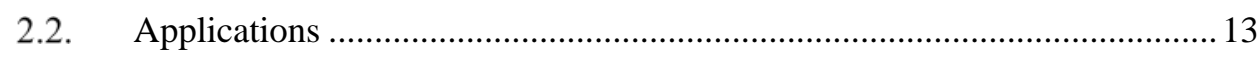

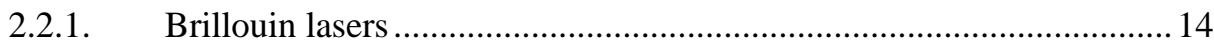

2.2.2. Distributed fiber sensors .................................................................. 14

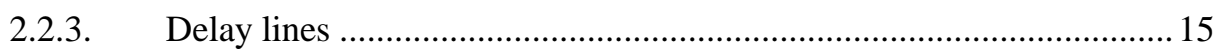

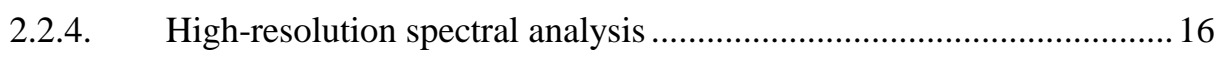

2.2.5. Optical processing of microwave signals.............................................. 16

CHAPTER 3 SBS-based All-optical Polarization Control ........................................... 17

3.1. Review of All-Optical Polarization Control............................................... 17

3.2. New SBS-based Polarization Control. ........................................................ 18

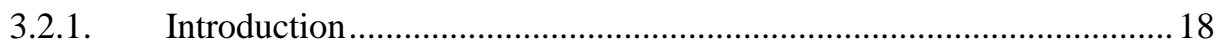




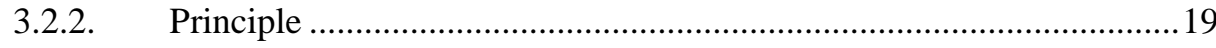

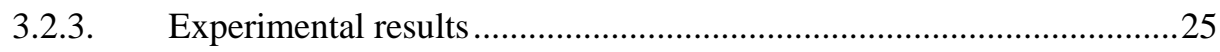

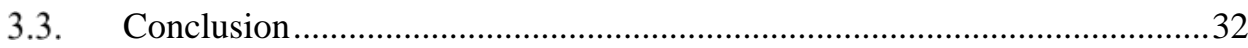

CHAPTER 4 SBS-based Photonic Microwave Filtering ...............................................34

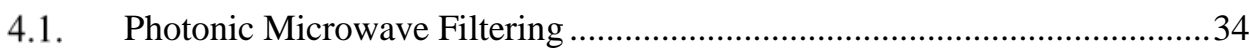

4.2. Review of SBS-based Photonic Microwave Filtering ....................................35

4.3. Photonic microwave filter with steep skirt selectivity based on stimulated

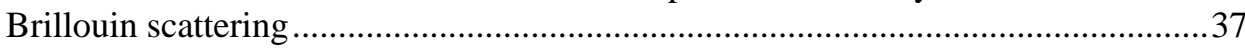

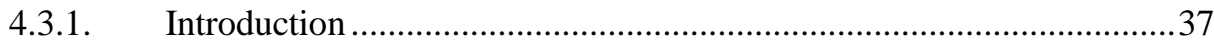

4.3.2. Photonic microwave filter architecture ……........................................37

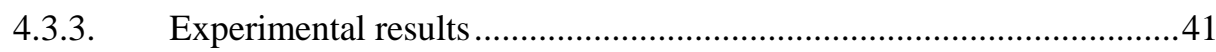

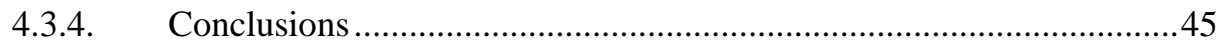

4.4. Brillouin Microwave Filter with enhanced Skirt Selectivity using a

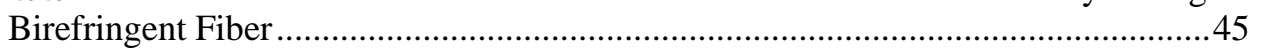

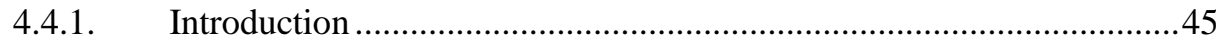

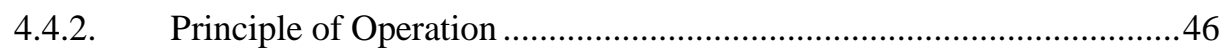

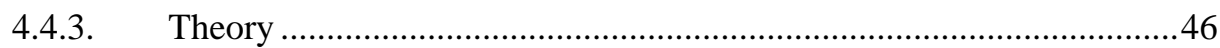

4.4.4. Experimental Results........................................................................50

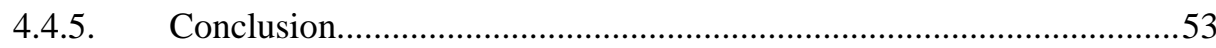

4.5. Wide tunable photonic microwave notch filter based on Brillouin polarization

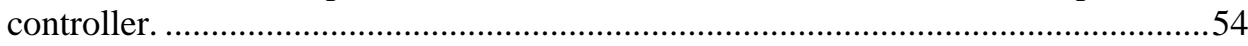

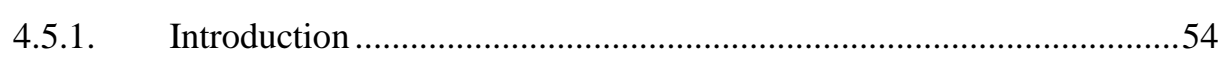

4.5.2. Principle of operation .......................................................................5

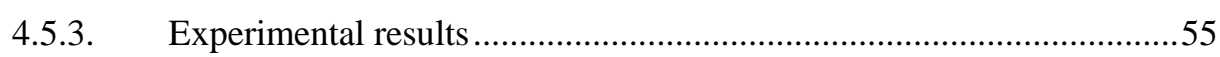

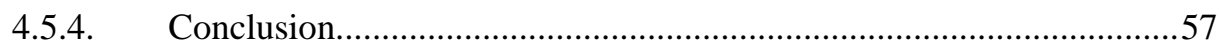

CHAPTER 5 Conclusions and future outlook...........................................................58

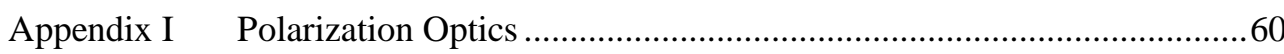

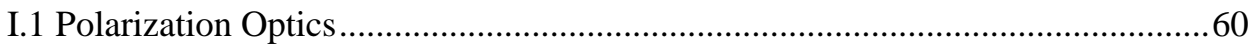

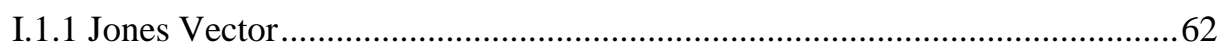

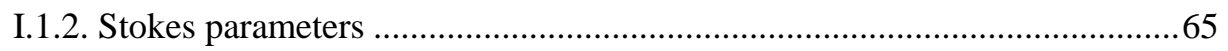




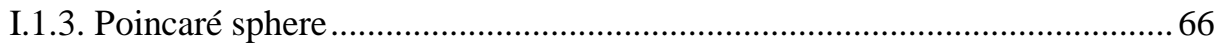

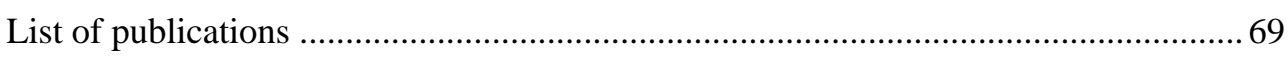

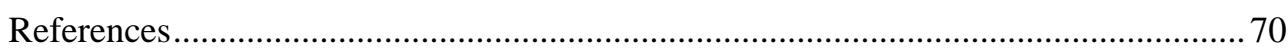




\section{Chapter 1}

\section{Introduction}

The application of optical nonlinear effects to solve engineering challenges is a fascinating field. Nonlinear phenomena are a powerful tool to address problems that are difficult to solve using linear methods.

Nonlinear optics is the study of the interaction of intense laser light with matter, in particular, the phenomena that result from the modification of the optical properties of materials by the presence of intense light. Although the history of linear optics can be traced back to the time of ancient Greeks, the nonlinear aspects of light started with the first observation by Peter Franken and his colleagues at the University of Michigan in Ann Arbor of second-harmonic generation, just one year after the discovery of the laser by Maiman in 1960.

Optical nonlinearities can be explained directly from Maxwell's equations. On a fundamental level, the origin of the nonlinear response is related to the harmonic motion of bound electrons under the influence of an applied field. In particular, the nonlinear effects arise when the polarization vector, $\boldsymbol{P}$, is not linearly proportional to the electric field, $\boldsymbol{E}$. This relation is usually approximated by a Taylor series. The response of any dielectric to light becomes nonlinear for intense electromagnetic fields.

Nonlinear phenomena can be grouped according to their origin as coming from the second-order or third-order susceptibility. Second-order susceptibility is zero in media that have inversion symmetry at the molecular level. $\mathrm{As}_{\mathrm{SiO}_{2}}$ is a symmetric molecule, second-order susceptibility vanishes for silica glasses and dominant nonlinear effects are third-order effects. These effects are originated from the nonlinear refraction index (Kerr effect) or from stimulated inelastic scattering.

Stimulated Brillouin Scattering, SBS, is an inelastic scattering effect that requires the lowest activation power of all non-linear effects in silica optical fibers. Leveraging the 
features of SBS to address engineering challenges will be the main aim of the present Thesis. Specifically, SBS will be studied to implement optical processing functionalities such as all-optical Polarization Control and Photonic Microwave Filtering.

The Thesis will be organized as follows. In the next Chapter, the fundamentals of SBS will be reviewed, focusing on the main concepts used through the Thesis. Chapter 3 will be focused on a new approach to provide light-by-light control of the state of polarization using SBS. Chapter 4 will be focused on studying new architectures for optical processing of microwave signals using SBS. In particular, new photonic microwave filters will be presented. Finally, conclusions and further research lines will be provided in Chapter 5. 


\section{Chapter 2}

\section{Fundamentals of stimu- lated Brillouin scatter- ing}

Stimulated Brillouin Scattering (SBS) [1], [2] is a nonlinear process that occurs in optical fibers. This effect manifests itself through the generation of a backpropagating Stokes wave that carries most of the input power, once the Brillouin threshold is reached. The process can be described as a nonlinear interaction among the pump and Stokes fields and an acoustic wave through electrostriction, i.e. changes in refraction index from material compression-distension. It is a stimulated inelastic scattering in which the optical field transfers part of its energy to the nonlinear medium.

This spontaneous interaction between a light signal and a carrier-deformation (acoustic) wave was theoretically predicted by León Brillouin [3] in 1922. However, the stimulated effect was not experimentally observed until 1964 by Chiao et al [4]. In optical fiber, SBS was first reported by Ippen an Stolen in 1972 [5] and shortly it was realized that SBS is the first nonlinear phenomenon appearing in silica optical fibers as optical power is increased.

The particular features of SBS have paved the way to its application in different fields, [6]. Among these can be highlighted Brillouin lasing, fiber sensors, SBS-based optical delay lines, high-resolution spectral analysis, enhancement of the SBS efficiency in Raman-pumped fibers, and microwave photonics.

In this chapter, the theoretical framework needed to describe SBS is presented and applications of this nonlinear effect are reviewed. 


\subsection{Theory of SBS}

Brillouin scattering is a nonlinear effect, inelastic scattering, caused by the imaginary part of a medium, in particular, it is mediated by acoustic phonons. The mechanism can be explained as the transformation of a photon from an incident light wave into a phonon and a scattered photon of slightly lower energy, i.e. downshifted in frequency ${ }^{1}$. Alternatively, it can be seen as a pump light creating a moving density grating through the effect of electrostriction from which it scatters in the backward direction. Electrostriction can be defined as the tendency of materials to become compressed in the presence of an electric field. Thus, light is changing the density of the medium. The frequency downshift of the Stokes wave can also be explained by the Doppler effect.

Brillouin scattering can occur spontaneously with signals of low optical power from the interaction with a thermally generated phonon field. In this configuration, only one laser beam (pump wave) is applied externally, and both the acoustic and Stokes fields grow from noise. The spontaneous Brillouin process of the scattered light is illustrated schematically in Figure 2.1.

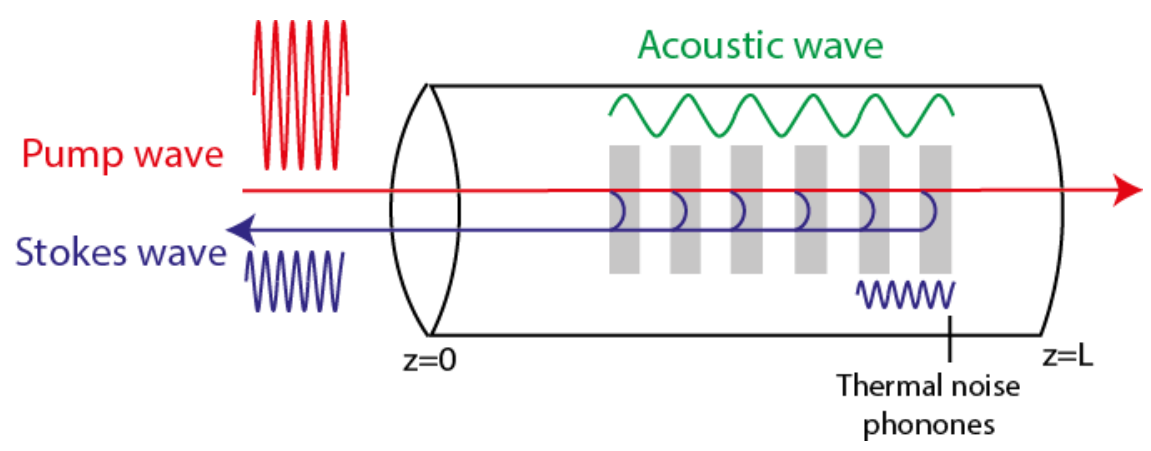

Figure 2.1 Schematic representation of the Spontaneous Brillouin scattering.

Beyond a certain pump power, the process becomes stimulated, i.e. it strongly depends on the pump power and the pump field firmly contributes to the phonon population. This is characterized by efficient energy conversion from the input light to the backscattered wave. The beating between pump and Stokes (at the downshifted Brillouin frequency)

${ }^{1}$ This wave is known as Stokes wave after physicist and mathematician George Stokes, who found the frequency downshift in the process of luminescence in the 19th century. 
wave creates a density change in Stokes wave. The density variation is associated with the power of the pump and Stokes waves and the mechanical conditions of the acoustic wave (optical medium parameters). External conditions such as local temperature, vibrations and strain induce changes in the fiber effective refractive index and sound velocity, which causes density variation in the Stokes wave.

Although the scattering cross section of the Stokes light is low, the attenuation in an optical fiber is also very low, making SBS a noticeable effect. The stimulated Brillouin scattered light is much more efficiently generated than the scattered light induced by spontaneous process. In fact, the response of the optical medium to the nonlinear interaction among the pump and Stokes fields induces exponential growth. The stimulated Brillouin process is illustrated schematically in Figure 2.2.

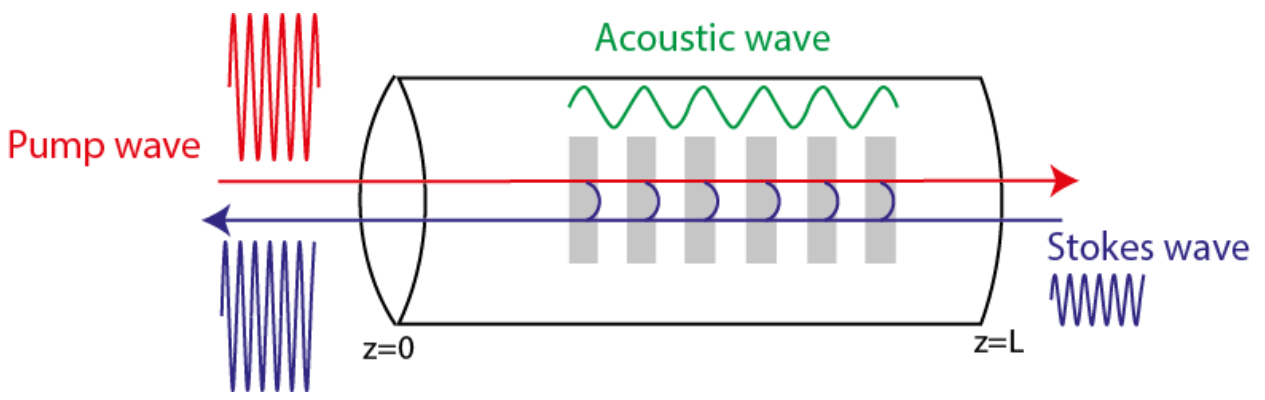

Figure 2.2 Schematic representation of the stimulated Brillouin scattering process.

The frequency shift of the Stokes wave depends on the scattering angle $\theta$ (angle between the pump and Stokes fields) and the wave vector $\boldsymbol{k}_{\boldsymbol{A}}$ of the acoustic wave. Because frequency shift is much smaller than pump frequency, the dispersion relation shows that the acoustic frequency is given by [1]

$$
v_{B}=v_{A}\left|k_{A}\right|=\frac{2 n_{p} v_{A}}{\lambda_{P}} \sin \left(\frac{\theta}{2}\right)
$$

The scattered light has a certain angular distribution, but the fiber geometry selects only two preferred directions-forward and backward. Equation 2.1 shows that the frequency shift is equal zero for forward scattering $(\theta=0)$ and is maximum for backscattering $\left(\theta=180^{\circ}\right)$. Forward Brillouin scattering in optical fibers is therefore very weak. Under these conditions, the SBS occurs only in the backward direction with a frequency shift, the so-called Brillouin frequency shift, given by

$$
v_{B}=\frac{2 n_{p} v_{A}}{\lambda_{P}}
$$


where $v_{A}$ is the acoustic velocity and $n_{p}$ is the effective mode index at the pump wavelength $\lambda_{P}$. Due to the energy and moment conservation during the SBS process, the frequencies of the three waves are related by

$$
f_{S}=f_{P}-v_{B}
$$

Equation 2.3

where $f_{P}$ and $f_{s}$ are the frequencies of the pump and Stokes, respectively. $v_{B}$ is related to the wave vector and acoustic velocity of the acoustic wave as can be seen in Equation 2.1. However, a second Stokes wave appears from scattering, the Anti-Stokes wave, at frequency

$$
f_{A S}=f_{P}+v_{B}
$$

Equation 2.4

Under steady-state conditions, applicable for a continuous wave (CW) pump, Boyd [2] provides analytical solutions to the coupled-intensity equations for a nonattenuating medium. These are presented below [1], [2],

$$
\begin{array}{lr}
\frac{d I_{P}}{d z}=-g_{B}(f) I_{P} I_{S}-\alpha I_{P} & \text { Equation } 2.5 \\
-\frac{d I_{S}}{d z}=+g_{B}(f) I_{S} I_{P}-\alpha I_{S} & \text { Equation } 2.6
\end{array}
$$

where $I_{P}$ and $I_{S}$ are the pump and Stokes intensities ${ }^{2}$, respectively, $\alpha$ is the fiber loss and $g_{B}$ is the SBS gain factor. These equations show that the SBS process is a net gain process for the Stokes wave and a loss process for the Anti-Stokes wave. The SBS gain/loss factor can be expressed as [2],

$$
\begin{array}{ll}
g_{B}(f)=g_{0} \frac{\left(\frac{\Delta v_{B}}{2}\right)^{2}}{\left(f_{P}-v_{B}-f\right)^{2}+\left(\frac{\Delta v_{B}}{2}\right)^{2}}-j \frac{g_{0}}{2} \frac{\Delta v_{B}\left(f_{P}-v_{B}-f\right)}{\left(f_{P}-v_{B}-f\right)^{2}+\left(\frac{\Delta v_{B}}{2}\right)^{2}} & \text { Equation 2.7 } \\
\alpha_{B}(f)=-g_{0} \frac{\left(\frac{\Delta v_{B}}{2}\right)^{2}}{\left(f_{P}+v_{B}-f\right)^{2}+\left(\frac{\Delta v_{B}}{2}\right)^{2}}+j \frac{g_{0}}{2} \frac{\Delta v_{B}\left(f_{P}+v_{B}-f\right)}{\left(f_{P}+v_{B}-f\right)^{2}+\left(\frac{\Delta v_{B}}{2}\right)^{2}} & \text { Equation 2.8 }
\end{array}
$$

where the Brillouin gain coefficient in the optical fiber is given by [1], [2]

\footnotetext{
${ }^{2}$ Optical intensity is related with the optical power by effective area, $P_{P, S}=I_{P, S} A_{\text {eff }}$
} 


$$
g_{0}=\frac{2 \pi \gamma_{e}^{2}}{n_{P} c \lambda_{P}^{2} \rho_{0} v_{A} \Delta v_{B}}
$$

Equation 2.9

This parameter depends on the material parameters: the electrostrictive constant of silica $\gamma_{\mathrm{e}}$, the material refractive index $n_{P}$, the speed of light in vacuum $c$, the pump wavelength $\lambda_{P}$, material density $\rho_{0}$ and $v_{A}$ the acoustic velocity. Meanwhile, $\Gamma_{B}$ is inversely proportional to the photon lifetime ( $\left.T_{B}\right)$ and it is related to the full width at half maximum (FWHM) of the gain Lorentzian spectrum as

$$
\Delta v_{B}=\frac{\Gamma_{B}}{2 \pi}
$$

The width of the Lorentzian SBS gain bandwidth is in the order of several tens of $\mathrm{MHz}$ for silica fiber in the $1550 \mathrm{~nm}$ band.

The Brillouin gain coefficient $g_{0}$ in Equation 2.9 shows that it is independent of the pump frequency.

Assuming no fiber loss, that both pump and Stokes waves maintain their state of polarization (SOP) along the fiber and if the pump power is spatially invariant, i.e. pump depletion is neglected, the solution to coupled-power Equation 2.5 and Equation 2.6 is given by [1], [2]

$$
P_{S}(0)=P_{S}(L) \exp \left(g_{B}(f) \frac{P_{P}(0) L_{e f f}}{A_{e f f}}\right)
$$

where $L_{e f f}$ is the effective interaction length, defined as $L_{e f f}=(1 / \alpha)(1-\exp (\alpha L))$. The Stokes wave, injected into the optical medium at $z=L$, experiences exponential growth as it propagates across the medium. Note that the Equation 2.11 assumes aligned polarization between the pump and the Stokes/signal. However, the effect is polarization sensitive and an efficiency coefficient related to the similarity between the polarization of the two counterpropagating signals has to be included in Equation 2.11. More details about this fact will be provided in the next section.

Finally, replacing Equation 2.7 in Equation 2.11, the Lorentzian magnitude and phase response due to the SBS gain process is given by the following equations

$$
\begin{aligned}
G(f) & =\exp \left(\operatorname{Re}\left(g_{B}(f) \frac{P_{P} L_{e f f}}{A_{\text {eff }}}\right)\right) \\
& =\exp \left(\frac{g_{0} P_{P} L_{e f f}}{A_{\text {eff }}} \frac{\left(\frac{\Delta v_{B}}{2}\right)^{2}}{\left(f_{P}-v_{B}-f\right)^{2}+\left(\frac{\Delta v_{B}}{2}\right)^{2}}\right)
\end{aligned}
$$

Equation 2.12 


$$
\begin{aligned}
\phi_{G}(f) & =\exp \left(\operatorname{Im}\left(g_{B}(f) \frac{P_{P} L_{e f f}}{A_{e f f}}\right)\right) \\
& =-\frac{g_{0} P_{P} L_{e f f}}{2 A_{e f f}} \frac{\Delta v_{B}\left(f_{P}-v_{B}-f\right)}{\left(f_{P}-v_{B}-f\right)^{2}+\left(\frac{\Delta v_{B}}{2}\right)^{2}}
\end{aligned}
$$

Note that Equation 2.12 and Equation 2.13 induce the SBS process at frequency $f_{P}-$ $v_{B}$. From Equation 2.4, it can be seen that a second Stokes wave appears from light scattering at frequency $f_{P}+v_{B}$, so-called SBS loss process and is written as

$$
\begin{aligned}
A(f) & =\exp \left(\operatorname{Re}\left(-g_{B}(f) \frac{P_{P} L_{e f f}}{A_{\text {eff }}}\right)\right) \\
& =\exp \left(-\frac{g_{0} P_{P} L_{\text {eff }}}{A_{\text {eff }}} \frac{\left(\frac{\Delta v_{B}}{2}\right)^{2}}{\left(f_{P}+v_{B}-f\right)^{2}+\left(\frac{\Delta v_{B}}{2}\right)^{2}}\right) \\
\phi_{A}(f) & =\exp \left(\operatorname{Im}\left(-g_{B}(f) \frac{P_{P} L_{e f f}}{A_{e f f}}\right)\right) \\
& =\frac{g_{0} P_{P} L_{e f f}}{2 A_{\text {eff }}} \frac{\Delta v_{B}\left(f_{P}+v_{B}-f\right)}{\left(f_{P}+v_{B}-f\right)^{2}+\left(\frac{\Delta v_{B}}{2}\right)^{2}}
\end{aligned}
$$

This process is characterized by an upshifted frequency and a negative SBS gain factor. The SBS gain/loss spectrum can be seen in Figure 2.3. It shows that the pump amplifies the signal at frequency $f_{S}$ through SBS gain process, while the pump attenuates the signal at $f_{A S}$.

The maximum value of the gain parameter, $G_{B}$, which is reached at frequency $f=f_{P}-$ $v_{B}$, is

$$
G_{B}=\frac{g_{0} P_{P} L_{e f f}}{A_{e f f}}
$$




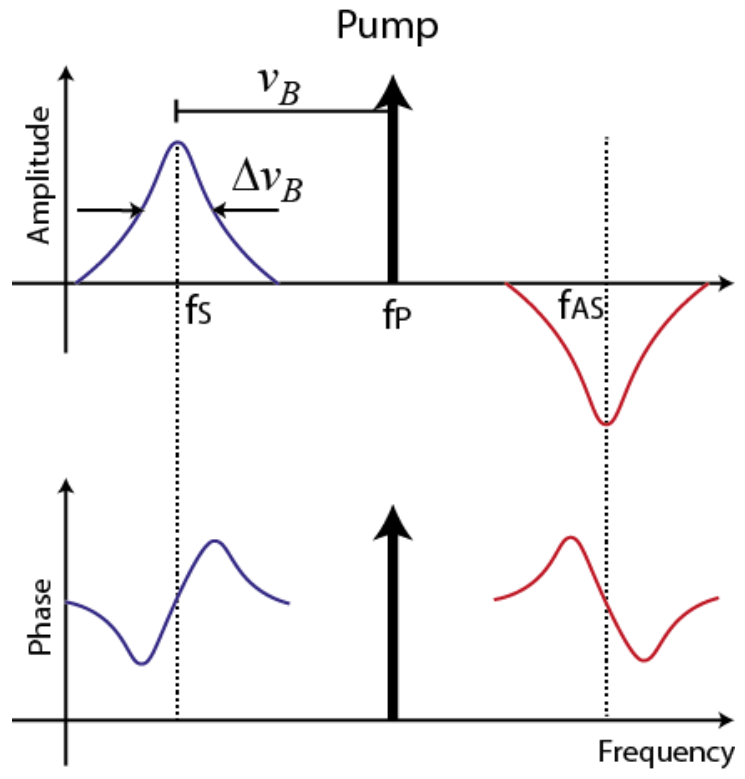

Figure 2.3. Amplitude and phase response of SBS

On the other hand, the phase shift induced by SBS is maximized when $f=f_{P}-v_{B}=$ $\pm^{\Delta v_{B} / 2}$. The maximum phase shift is,

$$
\phi_{B}=\mp \frac{g_{0} P_{P} L_{e f f}}{2 A_{e f f}}
$$

Equation 2.17

\subsubsection{Brillouin Threshold}

It is possible to estimate the Brillouin threshold from the spontaneous Brillouin process. If no Stokes wave is injected externally into the fiber in $z=L$, it grows exponentially from noise, propagating in backward direction, as Equation 2.11 shows. Note that, in this case, the value of $P_{S}(L)$ is the thermal noise power.

For the sake of simplicity, pump depletion can be neglected, i.e. it is assumed that pump power is spatially invariant before and after the outset of SBS, being solely considered the medium attenuation due to fiber loss, using $P_{P}(L)=P_{P}(0) \exp (-\alpha L)$ in Equation 2.11. From these considerations, Smith [7] defined Brillouin threshold assuming a nonfluctuating localized source model [8] as, 


$$
G_{B}^{t h}=\frac{g_{0} P_{P} L_{e f f}}{A_{\text {eff }}}=21
$$

Equation 2.18

The threshold value is obtained assuming typical values of standard single mode fiber [9]. Above this threshold, pump power saturates, limiting the power that can be transmitted through the fiber. For long fibers, the critical power is defined as the input pump power $(\mathrm{z}=0)$ at which the Stokes power becomes equal to the pump power at the fiber output $(\mathrm{z}=\mathrm{L})$, i.e. $P_{S}(0)=P_{L}(0) \exp (-\alpha L)$. Smith's model based on Equation 2.5 and Equation 2.6 defines the critical pump power as

$$
P_{P}^{c r i}(0)=\frac{21 A_{e f f}}{g_{0} L_{e f f}}
$$

Equation 2.19

Experimentally, lower threshold Brillouin gain factors have been obtained. For example, in [10] a value of $G_{B}^{t h}=19$ was found and in [11], using the same definition used in [7], it was found that Brillouin gain factor depends on fiber length, reaching a value of $G_{B}^{\text {th }}=$ 16 for the longest fibers $(\sim 30 \mathrm{~km})$.

\subsubsection{Polarization Effects on SBS}

Since SBS originates from an optical interference between pump and Stokes/signal waves, the SBS process is most efficient when optical fields are aligned. However, due to the nature of SBS, both pump and Stokes waves propagate in counterpropagation. Thus, both fields are aligned solely at a given point along the fiber.

In early experiments, Stolen [12] verified the polarization dependence of SBS in highly birefringent fiber, i.e. polarization maintaining fiber, PMF. Experiments showed that no SBS gain was observed when pump and signal waves were orthogonal. If both pump and Stokes waves are aligned, i.e. their Stokes vectors are identical, the SBS gain is reduced by a factor of 2 over scalar theoretical predictions [1].

On the other hand, in low birefringence fibers, Stolen interpreted that the SBS gain was independent of polarization. Later, Horiguchi et al. [13] demonstrated a polarization dependence of the SBS gain, reducing the SBS gain in a factor of $\sim 0.3$ for fibers from 1 to 26 km long.

In low birefringence fibers, the light SOP describes a rotation over the Poincare sphere [14], provided that the fiber length is much larger than the polarization beat length. However, the SOP of both pump and Stokes rotate in counter sense through fiber. Consequently, the overall SBS gain factor is reduced, depending on the SOP of input both pump and Stokes waves, and the birefringent properties of the fiber.

Van Deventer and Boot [15] investigated the polarization properties of the SBS amplification process in single-mode fibers, calculating the polarization dependence of the SBS 
gain and the SOP of both pump and Stokes waves by means of the Stokes representation [14] .

A simple method to take into account the polarization-sensitive nature of SBS is through an efficiency factor, $\zeta$, added to Equation 2.16. It will be $\zeta=1$ when the interaction between pump and Stokes waves is optimal.

The sense of the rotation of the pump SOP is opposite to that of the Stokes wave, but the orientation and ellipticity of the polarization ellipse remain the same [16]. Thus, for fibers without birefringence and linear pump polarization, the mixing efficiency is 1 when pump and Stokes waves have the same SOP and 0 when having orthogonal SOPs. For conventional low birefringence fibers the process is stochastic. Van Deventer and Boot concluded that in this case, the resulting mixing efficiencies for parallel and orthogonally polarized signals are respectively

$$
\begin{aligned}
& \zeta=\frac{2}{3} \\
& \zeta=\frac{1}{3}
\end{aligned}
$$

Equation 2.21

where $\zeta$ is the polarization coefficient. Equation 2.20 shows that $2 / 3$ of the pump power is used in the SBS process gain, reaching the maximum gain coefficient $G_{B}^{\max }$ when pump and Stokes have identical polarization, in their respective directions of propagation. Meanwhile for Equation 2.21, $1 / 3$ of the pump power is involved when pump and Stokes are orthogonal, reaching a minimum gain coefficient. With these considerations, the Equation 2.16 might be re-written as

$$
G_{B}=\zeta \frac{g_{0} P_{0} L_{e f f}}{A_{e f f}}
$$

Equation 2.22 shows that the SBS gain parameter in low birefringence fiber is smaller than the one reported in Equation 2.16. This fact also changes the SBS threshold value in Equation 2.18, and thus the critical pump power in Equation 2.19

$$
P_{P}^{c r i}(0)=\frac{21 A_{e f f}}{\zeta g_{0} L_{e f f}}
$$

Regarding spontaneous Brillouin scattering, when no signal wave is injected, there is a maximum amplification process from thermal noise [1], with the same SOP as that of the pump wave. At the same time, a second amplification process (minimum) appears with orthogonal polarization to the pump wave. The maximum and minimum SBS gain are always orthogonal. Thus, the relative input polarization of both pump and Stokes waves maximize or minimize the SBS gain coefficient $\left(G_{B}\right)$. 
Zadok et al. in [17] demonstrated that, in low birefringence fibers, the evolution of the signal SOP is controlled by the local interaction of the SBS, which drags the signal SOP toward that of the pump (in the SBS gain scenario). Thus, the output signal SOP converges to a particular point, i.e. it is rotated around a set of Stokes parameters, in the Poincare sphere as the pump power is increased.

Further, Zadok et al. determined that for any input SOP, the polarization coefficient is similar to that of [15], but not identical, since the polarization coefficient is only constant if the signal SOP is parallel/orthogonal to that of SOP pump. For the Brillouin attenuation process (SBS loss scenario), the anti-Stokes SOP is dragged toward a SOP perpendicular to that of the pump. Although, in this last case, experimental measurements of the SOP are difficult due to the attenuation produced in the signal and to the presence of spontaneous Brillouin.

\subsubsection{Pump depletion}

During the Brillouin process, the Stokes wave experiences exponential growth as it propagates across the optical fiber. When the Stokes wave has grown to an intensity comparable to that of the pump wave, the depletion of the pump wave occurs. Taking pump depletion into account, solving coupled-intensity Equation 2.5 and Equation 2.6 becomes a bit more complex. Anyway, it can also be solved both numerically and analytically.

\subsubsection{SBS model}

An analytical solution taking into account pump depletion but neglecting fiber loss was presented by Chen and Bao [18] ${ }^{3}$. This SBS model was employed through the Thesis and its application will be shown in several sections of this document, in particular, Sections $3.3,4.3$ and 4.4 .

The analytical expressions are given by,

$$
\begin{aligned}
& P_{P}(z) \\
& =\left[P_{P}(0)-P_{S}(0)\right] \frac{P_{P}(0) \exp \left\{\frac{g_{B}}{A_{e f f}}\left[P_{P}(0)-P_{S}(0)\right] L_{e f f}\right\}}{P_{P}(0) \exp \left\{\frac{g_{B}}{A_{\text {eff }}}\left[P_{P}(0)-P_{S}(0)\right] L_{e f f}\right\}-P_{S}(0)}
\end{aligned}
$$

\footnotetext{
${ }^{3}$ Other models base on neglecting loss attenuation have been proposed in the literature, e.g. [110].
} 


$$
\begin{aligned}
& P_{S}(z) \\
& =\left[P_{P}(0)-P_{S}(0)\right] \frac{P_{S}(0)}{P_{P}(0) \exp \left\{\frac{g_{B}}{A_{e f f}}\left[P_{P}(0)-P_{S}(0)\right] L_{e f f}\right\}-P_{S}(0)} \quad \text { Equation } 2.25
\end{aligned}
$$

With theoretical parameters described in the Table 2.1, a theoretical critical power of $53 \mathrm{~mW}$ can be calculated from Equation 2.23. It shows that, for this pump power $\left(P_{P}^{c r i}\right)$, the input Stokes wave has grown to a power comparable to that of the input pump power, as it was described in [2], [7], [11], which leads to a subsequent depletion. It shows that the Stokes power cannot grow further by increasing the input pump power due to depletion, reaching an input pump power limit.

The model defined by Equation 2.26 and Equation 2.27, with the general parameters given by Table 2.1 for a high nonlinear fiber, has been used to estimate the behavior of the SBS-based systems developed through the Thesis.

Table 2.1 Parameters for simulation

\begin{tabular}{|c|c|c|}
\hline Parameter & Value & Unit \\
\hline$L$ & 1 & $\mathrm{Km}$ \\
\hline$g_{0}$ & $7.19 \times 10^{-12}$ & $\mathrm{~m} / \mathrm{W}$ \\
\hline$A_{\text {eff }}$ & 11 & $\mu \mathrm{m}^{2}$ \\
\hline$v_{B}$ & 9.642 & $\mathrm{GHz}$ \\
\hline$\Delta v_{B}$ & $\sim 50$ & $\mathrm{MHz}$ \\
\hline$\lambda_{P}$ & 1548 & $\mathrm{~nm}$ \\
\hline$P_{n}$ & -55 & $d B m$ \\
\hline
\end{tabular}

\subsection{Applications}

Stimulated Brillouin scattering was originally considered to be relevant obstacle to optical communications based on optical fiber since it limits the maximum channel power that can be injected into an optical fiber [7]. Although present modulation speed make this no longer an issue, SBS still poses a limit to the maximum power that can be transmitted through fiber for an optical carrier. The optical power of a monochromatic wave at the output of an optical fiber increases proportionately until a threshold is reached. Beyond that level, the received power stays relatively constant while light scattering back toward the source increases dramatically. 
Despite this limitation, SBS has some unique features among nonlinear phenomena. These can be leveraged to develop photonic systems addressing a wide range of applications. Among these, the fields where Brillouin has attracted more attention are probably: lasing, fiber sensors, SBS-based optical delay lines, high-resolution spectral analysis and microwave photonics. Other applications include enhancement of other nonlinear effects such as Four-Wave Mixing [19].

In this section, the main applications of stimulated Brillouin scattering are briefly reviewed.

\subsubsection{Brillouin lasers}

Brillouin gain can be used to make lasers by placing the nonlinear medium inside a cavity. Brillouin lasers based on optical fiber were proposed as early as 1976 [20].

The mechanism is similar to any laser, with SBS providing the gain. The cavity is typically based on a ring or a Fabry-Perot geometry [1]. The feedback provided by the cavity reduces considerably the threshold. Thus, in Equation 2.23, the factor 21 can be reduced to $0.1-1$ depending on the reflectivity of the cavity. This allows Brillouin ring lasers to be pumped with laser diodes to make them simpler [21].

Smaller implementations of Brillouin lasers can be achieved using whispering gallery modes for the ring resonator if both pump and Stokes waves are at resonance wavelengths of the ring laser [22].

One particular feature of Brillouin lasers is that they show very narrow linewidth [23]. Recent publications have shown linewidths in the sub-Hz regime $(0.7 \mathrm{~Hz})$ [24].

Suggested applications include lasers with narrow linewidth, including CMOS compatible materials [25], microwave frequency generation and, especially, as sensitive laser gyroscopes [26]. Active laser gyroscopes use the fiber ring as a laser cavity. The rotation rate is determined by measuring the frequency difference between the counterpropagating laser beams.

\subsubsection{Distributed fiber sensors}

One of the main applications of SBS is in distributed sensing in large infrastructures. The aim is to replace thousands of point sensors with an optical fiber. The dependence of SBS on temperature and strain can be exploited to implement distributed sensors [27], [28].

As described by Equation 2.2, scattered light by Brillouin has a frequency shift, $v_{B}$, proportional to the local velocity of the acoustic wave $\left(v_{A}\right)$. This velocity, in turn, depends on the local density and tension of the material, which depends on its temperature and strain. The standard method, known as Brillouin optical time domain analysis (BOTDA), is based on pump-probe approach where a short pump pulse is launched into an end of 
the fiber used for sensing and a CW (continuous wave) probe signal into the other end. Sensing can be obtained from the measurement of the power of the probe signal with two similar schemes. If the probe wave is at the Stokes frequency, then energy flows from the pump to the Stokes wave providing Brillouin gain to this CW wave. Alternatively, Brillouin loss rather than Brillouin gain can be used to increase sensing length [29]. If the probe wave is at the anti-Stokes frequency, it provides energy to the pump wave (pulsed signal), and the detected CW signal experiences a Brillouin loss. Thus, the parameter $v_{B}$ at each fiber location can be determined from the measured time dependent $\mathrm{CW}$ signal over a wide range of frequency differences between the pump and probe. And from the Brillouin frequency shift, $v_{B}$, a map of the strain or temperature distribution along the entire fiber length can be derived. Further improvements on the basic BOTDA architecture have been presented (e.g. [30]).

Another approach for Brillouin-based distributed sensing is based on spontaneous scattering and it is known as Brillouin optical time domain reflectometry (BOTDR) [31], [32]. Other techniques include Brillouin Optical Correlation Domain Analysis/Reflectometry (BOCDA/BOCDR) [33], [34], [35].

These sensors can be applied in a wide range of scenarios where structural health monitoring is a need. It include fields such as civil structures and oil and utility industries, environmental monitoring, the aerospace industry, power generator monitoring and geotechnical engineering.

\subsubsection{Delay lines}

Optical delay lines can be considered one of the key building blocks in optical signal processing [36]. They are used for many different applications from microwave photonics (microwave signal processing, control of antenna arrays, etc.) to future optical buffers, and even in optical coherence tomography.

Different tunable optical delay lines have been proposed using fiber optics [37], [38], [39], or integrated devices [40], [41]. A different approach was proposed in 2005 when SBS was proposed as a method to implement optical delay lines based on the concept of slow light [42], [43]. SBS offers continuous dynamic tuning of the induced delay flexibility and the capability to shape the spectral resonance by controlling the shape of the optical pump since SBS response is the convolution of the Brillouin natural response and the shape of the pump. Despite these advantageous features, it can be said that the consensus in the engineering community is that, with present technology, there is a strong limitation on the applicability of SBS for optical delays given by the trade-off between the maximum achievable time delay and signal distortion. In particular, the delay-bandwidth product of linear slow light systems as SBS is close to 1, i.e. a pulse cannot be delayed more than its time width. 


\subsubsection{High-resolution spectral analysis}

This application relies on having a high-power pump that through SBS generates a tunable narrow filtering. By pumping SBS with an external cavity tunable laser source, the filter is swept along the spectral region of interest, giving the high-resolution optical spectrum [44], [45].

SBS offers interesting features over classic optical spectral analysis methods such as diffraction gratings, Fabry-Perot filters or heterodyne OSAs. The gain introduced by SBS greatly enhances the dynamic range of the measurement compared to passive filtering. The threshold imposed by SBS also helps cancel spurious signals of the local oscillator sidemodes and lineshape that produce measurement artifacts in heterodyne OSAs.

This approach has been commercially exploited by Aragon Photonics [46] which manufactures optical spectrum analyzers based on Brillouin effect (BOSA). This technology provides OSAs with high resolution (80 fm), good accuracy $(0.5 \mathrm{pm})$ and very high spurious-free dynamic range measurement.

\subsubsection{Optical processing of microwave signals}

Microwave photonics is a research topic focused on exploiting photonic technology for the generation, processing, control and distribution of microwave, millimeter-wave and THz-frequency signals [47], [48], [49], [50]. In particular, this field includes the use of photonics in a wide variety of applications such as antenna remoting [51], opticallycontrolled phased-array antennas (e.g. [52], [53], [54], [55]), photonic-assisted microwave measurement [56], optical processing of microwave signals and millimeter wave (e.g. [57], [58]) and THz signal generation [59].

Nonlinear effects, and SBS in particular, are very interesting in this research area to allow the implementation of some functionalities for microwave and millimeter-wave signals that are difficult to be obtained with pure electronic systems.

Originally, SBS gain was proposed to enhance the gain in radio over fiber links [60]. The narrowband nature of the Brillouin response in silica fibers is very attractive to process microwave signals [61]. For example, wideband phase shifters can be implemented using SBS to control the phase of the optical carrier [62]. In parallel, to the work on Brillouin lasing, briefly reviewed in Section 2.2.1, SBS can also be exploited to synthesize high purity microwave signals [63].

Among these applications, of the use of SBS in photonic microwave filtering has attracted also considerable interest. These research activities are reviewed in more detail in Section 4.2. 


\section{Chapter 3}

\section{SBS-based All-optical Polarization Control}

In this Chapter a new approach to polarization control based on exploiting SBS is presented. The state of the art on polarization control is briefly reviewed. The basic tools for polarization analysis are summarized in Annex I. Next, the new method for polarization control as well as experimental results showing its feasibility are presented.

\subsection{Review of All-Optical Polarization Control}

Many optical phenomena are sensitive to the state of polarization of the light. Thus, the ability to manage the state of polarization (SOP) is critical in different areas of photonics [64], [65]. New tools and techniques have evolved from advanced control of light polarization. In free-space optics, the development of polarization structured beams has opened new paths in both scientific and engineering applications. For example, spatiallydependent polarization beams for optical tweezers [66]. However, in optical fibers and integrated waveguides there are no tools offering similar potential for polarization structuring.

Currently, in-fiber polarization management is mainly performed using cascaded mechanically-controlled stress-induced birefringence in rotatable waveplates. The polarization control is induced by twisting [67] and/or bending [68],[69]. Twisting introduces circular birefringence and bending linear birefringence [64]. This approach was originally patented by C. LeFevre [68]. Another approach, pushed forward by General Photonics, is based on fiber implementations of a Babinet-Soleil compensator [70],

On the other hand, devices providing dynamic control of the state of polarization usually are based on electric or magnetic fields applied to an optical medium. These fields alter 
the polarization properties of the medium. In particular, this interaction is based on electro-optical devices exploiting the Pockels effect in $\mathrm{LiNbO}_{3}$ crystals [71] and magnetooptical components, such as Faraday rotators. However, all these are broadband devices and they cannot provide wavelength-selective polarization control analogous to the granularity that can be achieved in spatial dimensions with SLMs.

Nonlinear effects in optical fibers have been proposed as a method for all-optical control of the polarization of light [72]-[79]. Originally it was shown that photorefractive twobeam coupling can perform the transformation from any input SOP to the vicinity of a defined output SOP in what was known as polarization attraction [78], [79]. Later, polarization attraction was shown in other nonlinear systems, i.e. Four Wave Mixing (FWM) [8-10], Stimulated Brillouin Scattering (SBS) [73], [74] and Stimulated Raman scattering (SRS) [72]. Attractors, however, do not behave as a perfect polarizer since they pull input SOPs toward the vicinity of some point but not exactly to a given point on the Poincaré sphere.

Since SBS originates from an optical interference between pump and signal waves. SBS is most efficient when these electric fields are aligned. Due to the large difference in gain between the maximum and minimum states, the SOP of an arbitrarily polarized input wave is closely aligned with that SOP of the maximum gain at the fiber output. Therefore, the polarization sensitive gain of the SBS can be used to implement a functionality similar to a polarizer but usually providing polarization-dependent gain instead of loss, i.e. SBS in the undepleted pump regime may be modeled as a pseudo-linear partial polarizer [17].

SBS polarization pulling can be enhanced using two orthogonally polarize pumps [74], which are separated in frequency by twice the Brillouin shift, where an amplifying one and attenuating one. The SOP of the signal wave is drawn toward the conjugate of the higher frequency pump. Meanwhile the lower frequency pump strengthened the pulling, due to it repels the signal SOP from the orthogonal state and therefore the dual-pump configuration generates a higher change in the SOP signal with a more modest maximal gain.

\subsection{New SBS-based Polarization Control.}

\subsubsection{Introduction}

A new approach for polarization rotation through nonlinear effects is presented in this section. Instead of a nonlinear polarizer, here we show that polarization control can be achieved exploiting nonlinear optics. In particular, we investigate the implementation of an all-optical analogue of a polarization controller based on a variable linear retarder (such as a wave plate) with fixed orientation plus one variable circular retarder (optical rotator). The variable retarders rely on exploiting the Brillouin-induced birefringence in 
optical fibers to control the phase shift between orthogonal components of the SOP while conserving the energy of the signal though a combination of phase shifting and gain/loss compensation.

\subsubsection{Principle}

Nonlinear polarization control is achieved by inducing an optically-controlled phase retardance between eigenmodes in an optical fiber through SBS. Since Brillouin interaction is originated from an interference between counterpropagating waves through electrostriction, its efficiency is polarization dependent, i.e. the gain (loss) coefficient is maximum when the pump and signal electric fields rotate describing the same ellipses with opposite senses. From this polarization dependence, a controlled phase shift between eigenmodes can be introduced using SBS if the pump is aligned with one eigenmode of the signal. Given the short coherence lifetime of hypersonic phonons in fiber, the Brillouin response bandwidth can be tuned from $30 \mathrm{MHz}$ to tens of $\mathrm{GHz}$, allowing the independent control of the SOP of individual optical signals in DWDM scenarios.

The natural response of SBS associates phase shift with a gain (Stokes) / loss (antiStokes) response, as shown in Figure 3.1 (blue/red responses). However, it is possible to combine Brillouin gain and loss resonances to compensate each other in amplitude while adding the phase shifts provided by both responses, as shown in Figure 3.1 (green responses). The figure shows two pump waves at frequencies $f_{0} \pm f_{p}$ which are used to combine the gain/loss responses, where $f_{0}$ and $f_{p}$ are the signal and pump frequencies, respectively.

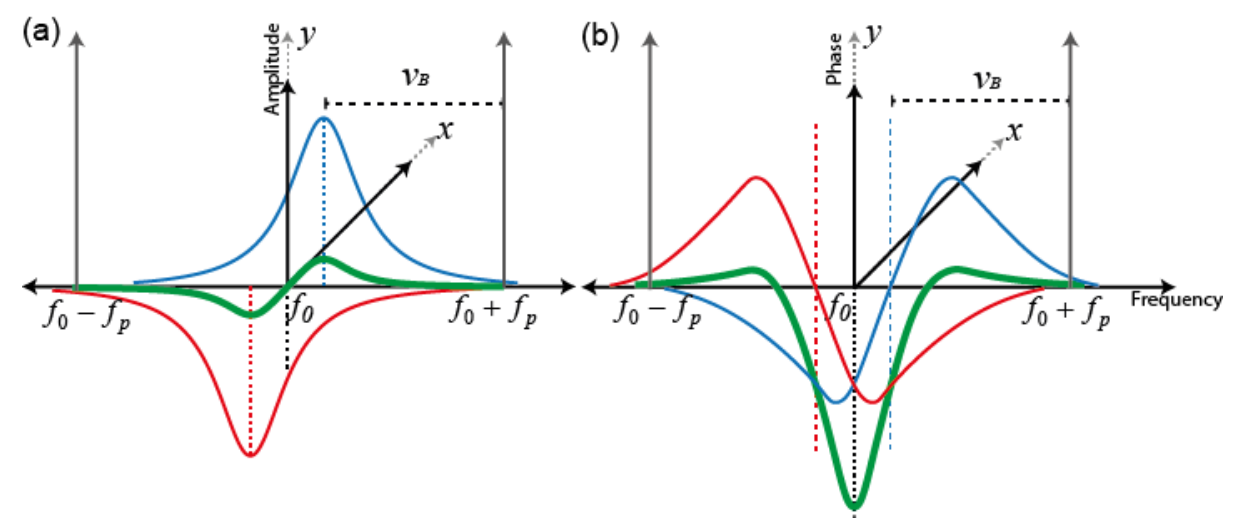

Figure 3.1 (a) SBS amplitude response showing the gain (blue), loss (red) and combined gain+loss response (green); (b) SBS phase response showing the gain (blue), loss (red) and combined gain+loss response (green) 
While maintaining the amplitude of the signal minimally affected, this approach allows the enhancement of the phase response. This is analogous to an optical all-pass filter. SBS-based all-pass filters have been proposed to control the phase of optically-modulated RF signals [62], [80]. From this point of view, the proposed method can be seen as an evolution from the Loayssa SBS-based microwave phase retarder.

The phase retardance $(\Gamma)$ between orthogonal polarization eigenmodes of the signal can be dynamically controlled by changing the pump power or the pump offset frequency. However, the use of pump power is more convenient due to its simplicity for implementation.

A polarization controller based on two variable retarders with fixed orientations can be built using SBS, as shown in Figure 3.2 (a).
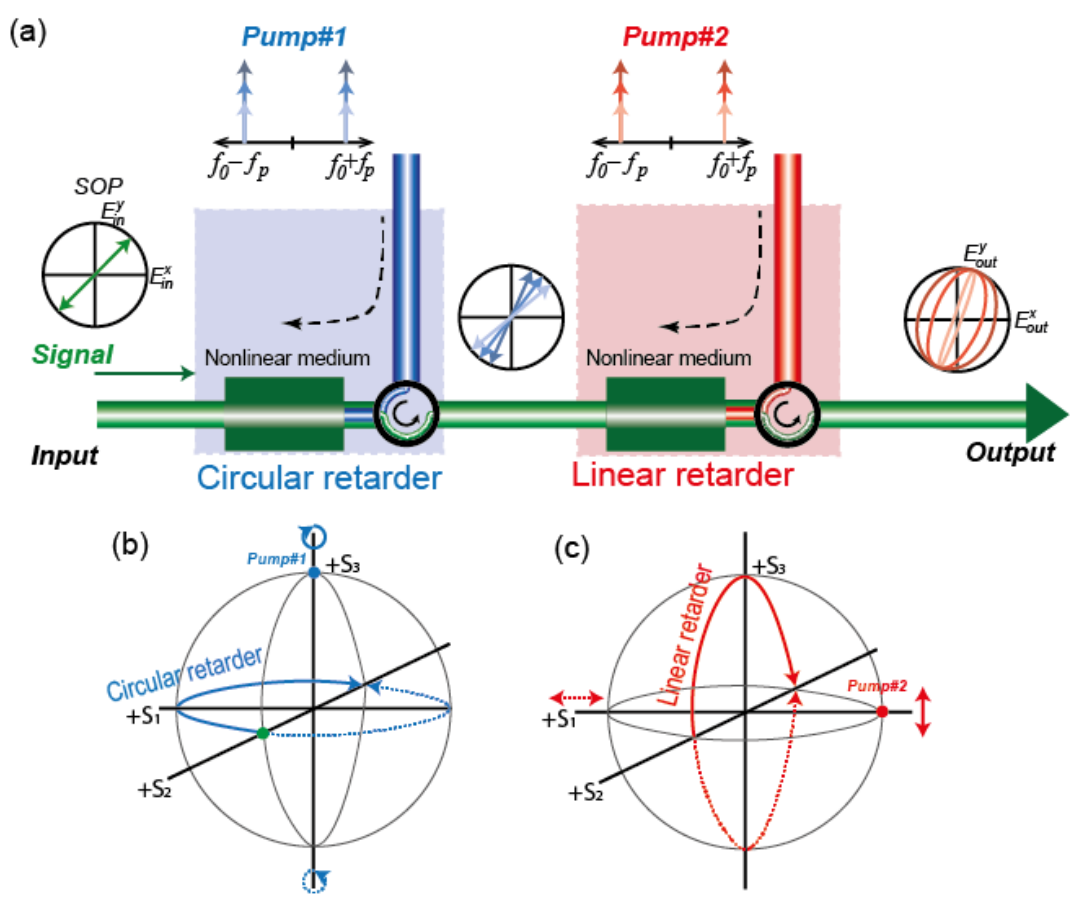

Figure 3.2 (a) Concept of the nonlinear polarization controller based on two independently controlled variable waveplates: a circular retarder and a linear retarder. The SOP of an incoming optical signal at $f_{0}$ with, for example, linear polarization at $45^{\circ}$ is altered by a pair of pump signals, gain and loss, respectively, at $f_{0}-f_{p}$ and $f_{0}+f_{p}$ with circular polarization for the circular retarder, and a second pair of pump at $f_{0}-f_{p}$ and $f_{0}+f_{p}$ with linear polarization for the linear retarder. (b) Representation of the polarization rotation on the Poincaré sphere for the ideal circular (blue) and (c) linear (red) retarders. 
Brillouin allows the implementation of arbitrary birefringent elements where the pump SOP defines the birefringence type, i.e. the plane of polarization rotation on the Poincaré sphere while the pump power controls the angle of rotation on this plane. To perform a circular retarder (Pump \#1), the pump polarization has to be circular. Its induced polarization rotation is shown in Figure 3.2(b) (blue). In particular, right-handed circular polarization rotates the signal SOP clockwise. Whilst the second stage, with Pump \#2 having a linear horizontal SOP, performs a linear retarder, as shown in Figure 3.2(c).

The Brillouin-induced retardance can be calculated taking into account that SBS in low birefringence single-mode fibers can be viewed as a polarization-dependent gain (loss), where there are two orthogonal input SOPs, each one showing a different gain (loss) [17]. The gain of the input SOP with the maximum gain coefficient is twice the minimum one, being $2 / 3$ and $1 / 3$ of the gain in a fiber with low birefringence. Thus, in a birefringent fiber, the Brillouin induced phase shift is given from Equation 2.13 and Equation 2.15 ,

$$
\varphi_{\text {max } / \text { min }}=\frac{\zeta g_{0} P_{p} L_{e f f}}{2 A_{e f f}} \frac{\Delta v_{B}\left(f_{p}-v_{B}\right)}{\left(f_{p}-v_{B}-f\right)^{2}+\left(\frac{\Delta v_{B}}{2}\right)^{2}}
$$

where $P_{p}$ is the pump power, $g_{0}$ is the Brillouin gain factor, $A_{\text {eff }}$ is the effective area, $L_{e f f}$ is the effective interaction length of the fiber, $\Delta v_{B}$ is the Brillouin gain bandwidth of the gain spectrum and $v_{B}$ is the Brillouin frequency shift, and the term $\zeta$ is $2 / 3$ for the maximum gain/loss and $1 / 3$ for the minimum gain/loss in birefringent fiber. The maximum Brillouin phase shift corresponds to $f_{p}-v_{B}= \pm \Delta v_{B} / 2$. Therefore, the phase retardance induced by the SBS phase shift Equation 3.1 in a birefringent fiber is,

$$
\Gamma=2 \varphi_{\text {max }}-2 \varphi_{\text {min }}=\frac{g_{0} P_{p} L}{3 A_{\text {eff }}} \frac{\Delta v_{B}\left(f_{p}-v_{B}\right)}{\left(f_{p}-v_{B}\right)^{2}+\left(\frac{\Delta v_{B}}{2}\right)^{2}}
$$

It can be seen in Equation 3.2 that despite the combination of gain and loss phase responses, fiber birefringence reduces the maximum phase retardance that can be induced by using SBS to the phase shift induced by a single SBS gain response in a nonbirefringent fiber. Therefore, the Jones matrix of the nonlinear polarization controller is given from Equation I.10 and Equation I.12, 


$$
\begin{aligned}
& J_{S B S}=M_{2} M_{1} \\
& =\left(\begin{array}{cc}
G_{\min } A_{\min } \cdot\left(\cos \left(\frac{\Gamma_{2}}{2}\right)-j \sin \left(\frac{\Gamma_{2}}{2}\right) \cdot \cos (2 \delta)\right) & G_{\max } A_{\max } \cdot \sin \left(\frac{\Gamma_{2}}{2}\right) \cdot \sin (2 \delta) \\
G_{\min } A_{\min } \cdot \sin \left(\frac{\Gamma_{2}}{2}\right) \cdot \sin (2 \delta) & G_{\max } A_{\max } \cdot\left(\cos \left(\frac{\Gamma_{2}}{2}\right)-j \sin \left(\frac{\Gamma_{2}}{2}\right) \cdot \cos (2 \delta)\right)
\end{array}\right) \cdot \text { Equation } 3.3 \\
& \left(\begin{array}{rr}
G_{\min } A_{\min } \cos \left(\Gamma_{1} / 2\right) & G_{\max } A_{\max } \sin \left(\Gamma_{1} / 2\right) \\
-G_{\min } A_{\min } \sin \left(\Gamma_{1} / 2\right) & G_{\max } A_{\max } \cos \left(\Gamma_{1} / 2\right)
\end{array}\right)
\end{aligned}
$$

where Jones matrices, $M_{1}$ and $M_{2}$ correspond to the linear and circular retarder, respectively. $\Gamma_{1}$ is the retardance induced by circular birefringence (Pump \#1) and $\Gamma_{2}$ by the linear birefringence (Pump \#2). $\delta$ is the azimuth angle of the fast axis of the linear birefringence defined by the SOP of Pump \#2. This angle defines the orientation of the plane of rotation for the linear retarder.

If the gain and loss amplitude responses perfectly compensate each other, i.e. $\left|G_{\max }\right|$. $\left|A_{\max }\right| \cong 1$, and $\left|G_{\min }\right| \cdot\left|A_{\min }\right| \cong 1$, polarization control could be achieved while preserving the energy of the incoming signal.

For constant pump power, retardance can be controlled with the frequency shift between Stokes and pump. The maximum retardance is obtained with frequencies $f_{p 1}=v_{B}+$ $\Delta v_{B} / 2$ and $f_{p 2}=v_{B}-\Delta v_{B} / 2$. Figure 3.3 (a) and (b) show the evolution of the signal SOP when Pump\#1 has right circular SOP at frequency $f_{p 1}$ or $f_{p 2}$, respectively. These frequencies introduce an inverted Brillouin phase shift. Meanwhile, Figure 3.3 (c) and (d) correspond to Pump\#1 with left circular SOP at frequency $f_{p 1}$ or $f_{p 2}$, respectively.

As shown in Figure 3.3 (a), if the Brillouin gain parameter, $G=\zeta g_{0} P_{p} L /\left(A_{e f f}\right)$, induced by pump wave at $f_{p 1}$, is high, a significant amount of spontaneous Brillouin noise might be generated. However, this contribution can be considerably reduced using an additional pump wave, in Pump\#1, with frequency $f_{p 2}$ and orthogonal SOP. The evolution of the signal SOP in this case is shown in Figure 3.3 (d). Each orthogonal pump wave, at $f_{p 1}$ and $f_{p 2}$, induce a rotation in the same direction (inverted Brillouin phase shift), doubling the total retardance induced over the signal while suppressing the generation of spontaneous Brillouin noise. This is the approach followed in the block diagram shown in Figure 3.4. 


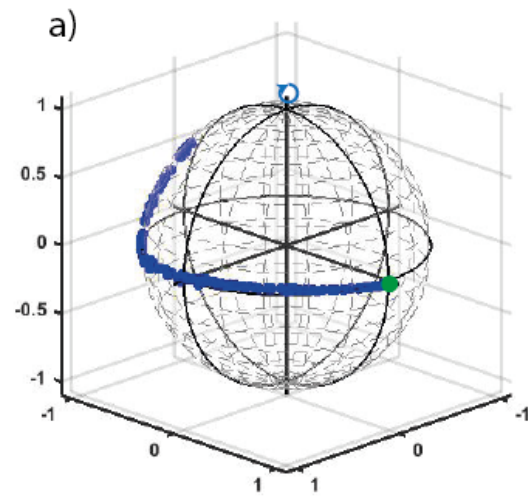

c)

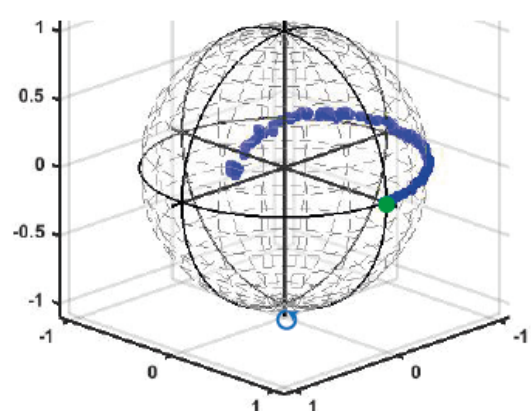

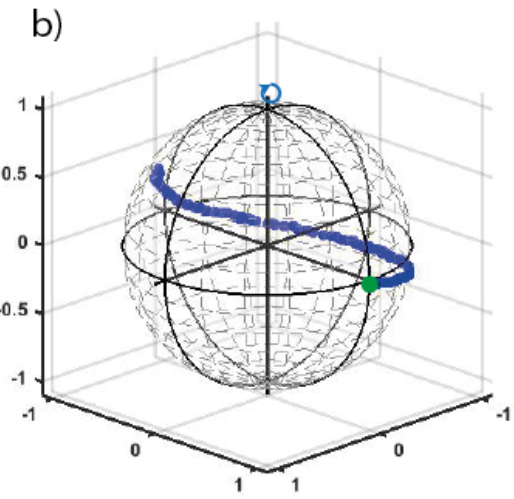

d)

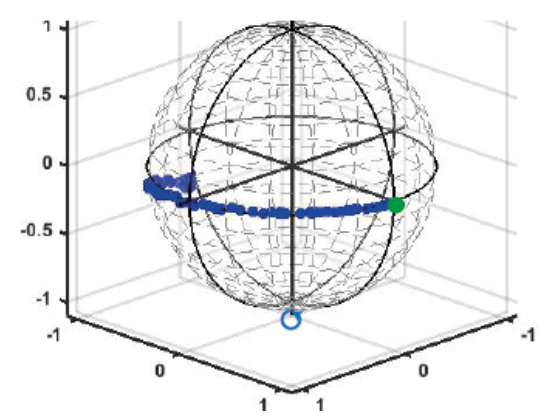

Figure 3.3 Poincaré sphere representation of the evolution of the output signal polarization over a circular retarder for an input signal with linear polarization at $45^{\circ}$ (Jones vector $\left([1 / \sqrt{2}(1,1)]^{T}\right)$ ) and pump SOP is: (a) Right circular at $f_{p 1}$, (b) Right circular at $f_{p 2}$, (c) Left circular at $f_{p 1}$, (d) Left circular at $f_{p 2}$.

The system shown in Figure 3.4 allows the rotation to an arbitrary output SOP by the combination of two pump pairs with orthogonal polarizations for each wave retarder. Each pump pair introduces a retardance up to $\pm \pi$ in the Stokes space. Each orthogonal pump wave, at frequency $f_{p 1}$ and $f_{p 2}$ induce a rotation in the same direction. The maximum retardance can be obtained if the two orthogonal pumps are at frequencies $f_{p 1}$ and $f_{p 2}$.

For the circular retarder, if the pump at $f_{p 1}$ and $f_{p 2}$ have a right and left circular SOP, respectively, the signal SOP will rotate to the West on the Poincaré sphere, as shown in the inset of Figure 3.4 (blue solid arrow). The signal SOP can be rotated to the East as shown in Figure 3.4 (blue dotted arrow) if frequencies $f_{p 1}$ and $f_{p 2}$ are swapped, such that $f_{p 1}=v_{B}-\Delta v_{B} / 2$ and $f_{p 2}=v_{B}+\Delta v_{B} / 2$. For the linear retarder, the rotation is 
obtained in the same way but the SOPs of the pump waves are linear at $90^{\circ}$ and $0^{\circ}$ for $f_{p 1}$ and $f_{p 2}$, respectively.

The polarization modulation speed of the system is restricted by the propagation velocity in the nonlinear medium. For a fiber length of $1 \mathrm{~km}$, the system will have a polarization modulation speed of approximately $200 \mathrm{kHz}$. However, the use of chalcogenide fiber [81] or photonic integrated devices [82], would enhance considerably the polarization modulation bandwidth up to the $\mathrm{GHz}$ range although another limitation arises from the time needed by phonons to be generated..

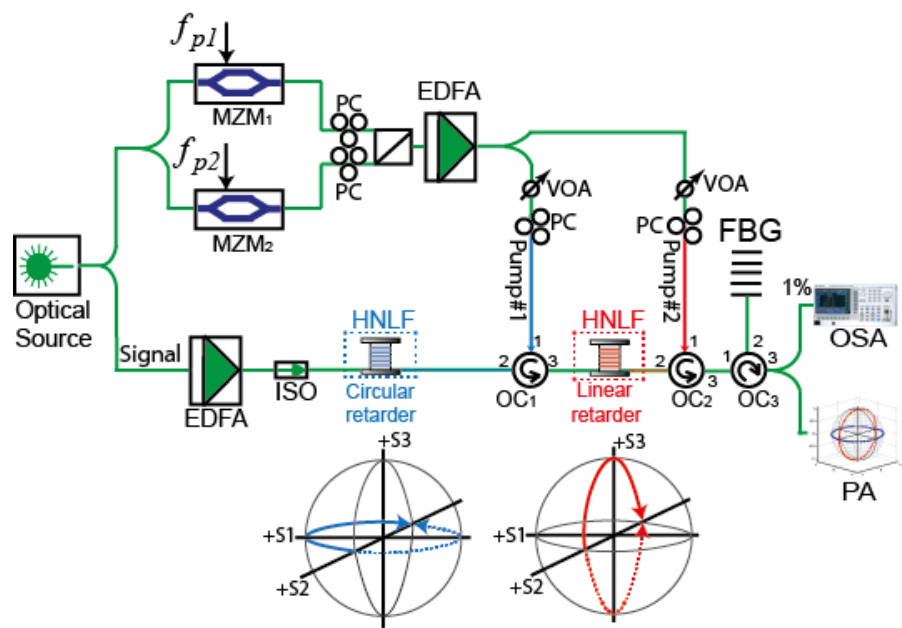

Figure 3.4 Block diagram of a nonlinear all-optical polarization controller made of one circular retarder plus one linear retarder. HLNF: Highly nonlinear fiber. OC: Optical circulator; ISO: Isolator. VOA: Variable Optical Attenuator. PC polarization controller. FBG: Fiber Bragg grating. OSA: Optical spectrum analyzer. PA: Polarization analyzer.

The experimental setup used to demonstrate the feasibility of nonlinear polarization control using SBS is the one shown in Figure 3.4. In particular, two Brillouin polarization control stages are implemented: a circular and a linear retarder. An optical signal is split into two paths: the upper one is used to generate the pump waves while the lower one experiences polarization rotation. The pump is generated by two x-cut Mach-Zehnder modulators ( $\mathrm{MZM}_{1}$ and $\left.\mathrm{MZM}_{2}\right)$ at minimum transmission bias (MITB) and fed by two microwave oscillators with frequencies $f_{p 1}(9.607 \mathrm{GHz})$ and $f_{p 2}(9.677 \mathrm{GHz})$. An optical circulator $\left(\mathrm{OC}_{1}\right)$ directs Pump\#1 toward the circular retarder, made of 1-km highly nonlinear fiber (HNLF). Another optical circulator $\left(\mathrm{OC}_{2}\right)$ is used to inject Pump\#2 to the linear retarder, where the nonlinear medium is another HLNF. A fiber Bragg grating (FBG) in reflection mode (bandwidth of $12.5 \mathrm{GHz}$ ) is used to filter out backward residual 
pump waves. Polarization rotation is done by adjusting the pump power through the VOAs.

\subsubsection{Experimental results}

Figure 3.5(a) shows the evolution of the signal SOP at the output of the circular retarder when the power of Pump\#1 is changed from $0.08 \mathrm{~mW}$ to $12.6 \mathrm{~mW}$. The retardance has been measured using the Poincaré sphere method [83]. It can be seen that a full rotation $(2 \pi)$ on the equator of the Poincaré sphere can be achieved. Figure 3.5(b) shows the evolution of the measured retardance with pump power as well as the output DOP. It can be observed that, as given by Equation 3.2, retardance varies linearly with pump power.

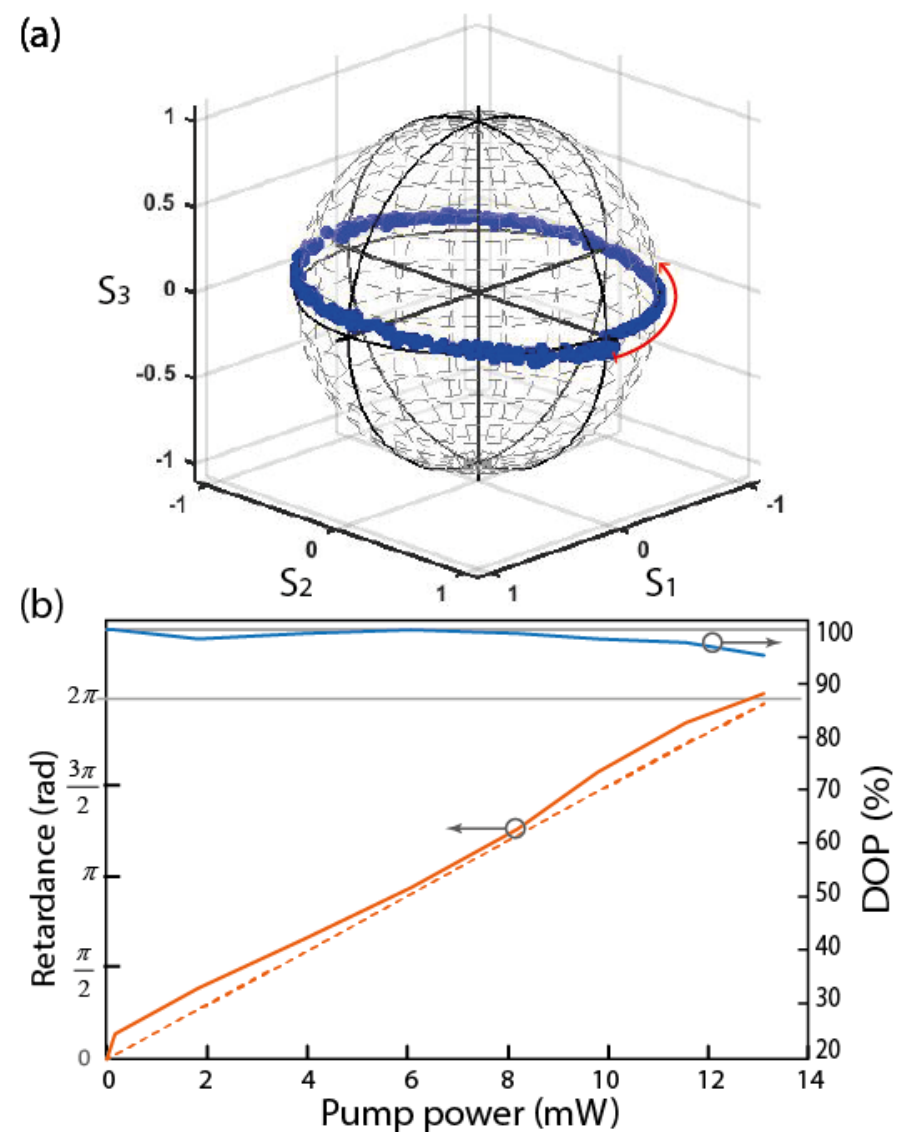

Figure 3.5 Experimental results for a $0.16 \mathrm{~mW}$ input signal. (a) Poincaré sphere representation of the evolution of the output signal polarization over a circular retarder for an input signal with linear polarization at $45^{\circ}$ (Jones vector $\left.\left([1 / \sqrt{2}(1,1)]^{T}\right)\right)$. (b) (Left) Retardance induced as a function 
of pump power for the circular retarder: experiment (solid orange) and theory (dotted orange); (Right) Evolution of the signal Degree of Polarization (DOP) for the circular retarder a function of pump power.

Given the nonlinear nature of the polarization control method, the effect of the power of the input signal on the behavior of the polarization converter has been experimentally analyzed. Figure 3.6 shows, for the circular retarder, the retardance variation as a function of pump power for different input signal powers. It can be seen that the induced retardance is remarkably similar in all cases. A maximum change on retardance can be observed for a pump power of $8.3 \mathrm{~mW}$, with a standard deviation of the retardance of $4.6^{\circ}$.

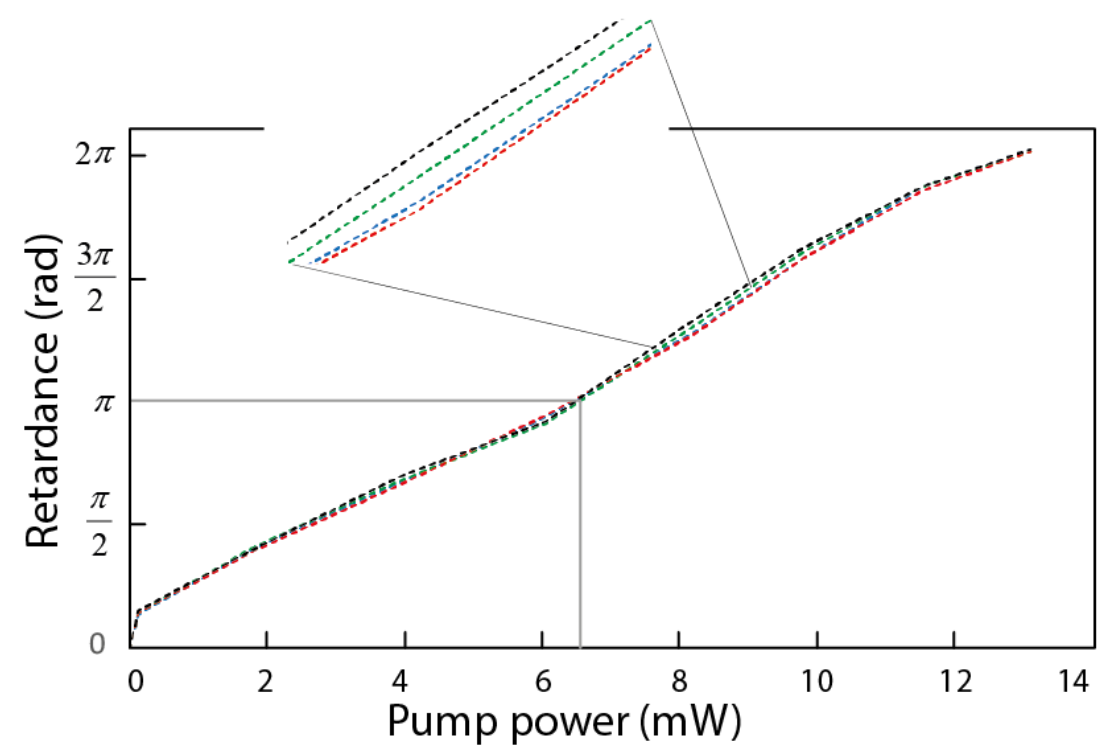

Figure 3.6 Retardance versus pump power for a Brillouin circular retarder for signals of: $0.63 \mathrm{~mW}$ (dotted black), $0.16 \mathrm{~mW}$ (red dotted), $0.08 \mathrm{~mW}$ (dotted green) and $0.04 \mathrm{~mW}$ (dotted blue).

However, there is no need for a full $2 \pi$ retardance change since the sense of the rotation on the Poincaré sphere can be tuned by swapping frequencies between $f_{p 1}$ and $f_{p 2}$. Thus, complete rotation of the SOP can be achieved, as shown in Figure 3.7. For a retardance smaller than $\pi$, the DOP remains above $98 \%$. 


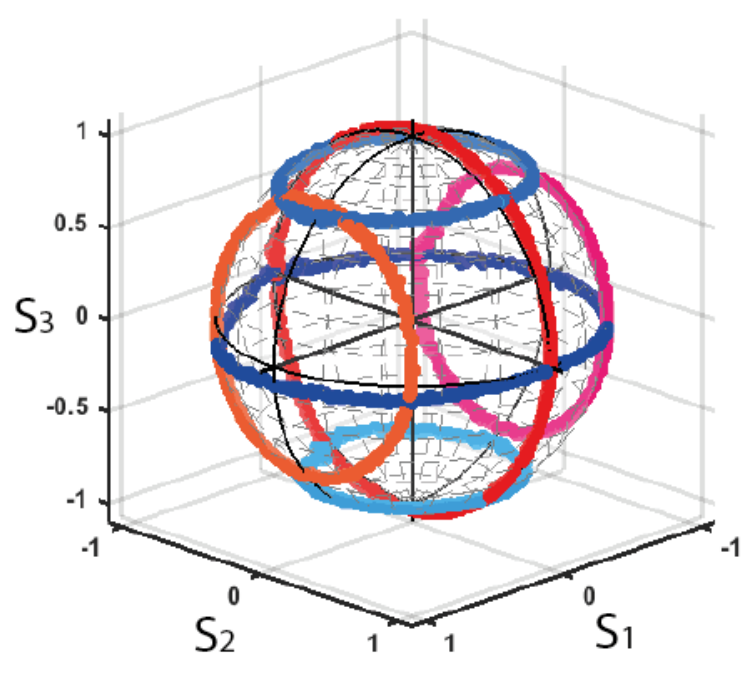

Figure 3.7 Rotation of the signal SOP induced by the Brillouin-based circular and linear retarders.

Due to the combination of gain and loss responses, Brillouin polarization rotation induces only slight changes on the signal power in the range of pump powers that are needed to reach any point on the Poincaré sphere. Figure 3.8 (a) shows measurements of the SBS-induced retardance for an input signal of $0.08 \mathrm{~mW}$ as a function of pump power and the variation of the power of the signal for the circular (blue solid and dotted) and linear (red solid and dotted) retarders. Figure 3.8 (a) shows that the nonlinear variable retarder is able to provide any kind of birefringence with the same efficiency. It can be seen that the same pump power induce the same retardance for the linear and the circular retarder. Whereas the retardance grows with pump power, following a trend similar to the behavior of the SBS gain, the power of the signal experiences only a slight variation. In Figure 3.8 (a), the variation of the insertion loss is $1.2 \mathrm{~dB}$. The action of the circular retarder is equivalent to a rotation $\left(M_{1}\right.$ matrix) of the sphere about the polar diameter, and through an angle equal to the phase retardance that the SBS inserts between its eigenmodes, i.e. the sphere's rotation angle (Figure 3.8 (b)). 
a)

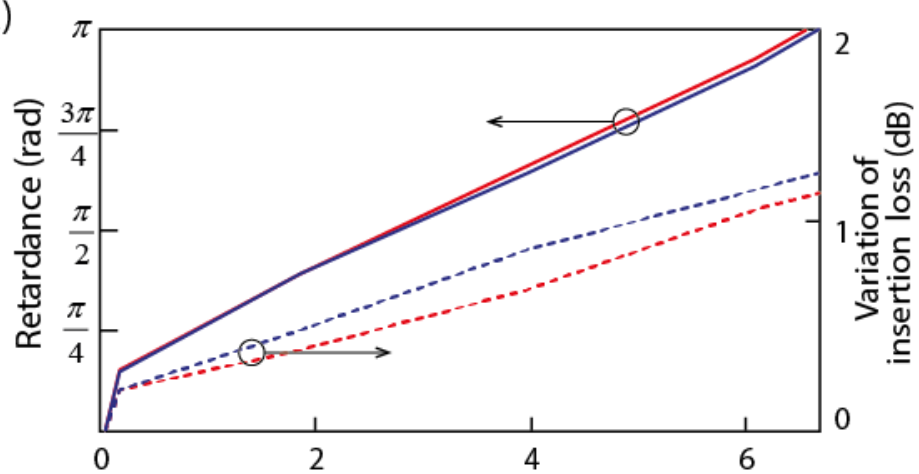

b)

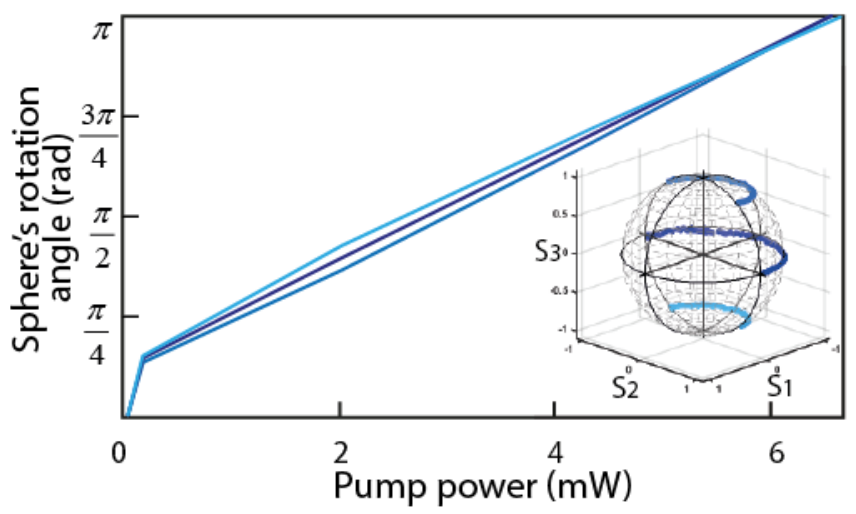

Figure 3.8 (a) (Left) Retardance induced as a function of pump power for the circular (blue solid) and linear (red solid) retarder for input signal with linear SOP at $45^{\circ}$ (Jones vector

$\left.\left([1 / \sqrt{2}(1,1)]^{T}\right)\right)$. (Right) Variation of the output signal power versus pump power. (b) Rotation angle for input signal with ellipticity of 0.5 (Jones vector $[1 / \sqrt{2}(1, \exp (j \pi / 4))]^{T}$,

$$
\left.[1 / \sqrt{2}(1, \exp (-j \pi / 4))]^{T}\right) \text {. }
$$

The effect of the power of the input signal on the loss of the polarization converter has also been analysed. Figure 3.9 provides measurements of the converter insertion loss for different polarization conversions and signals with different input power. It can be seen that for input signals with moderate power (smaller than $0.16 \mathrm{~mW}$ ) and for pump powers smaller than $7 \mathrm{~mW}$, which induce retardance changes of $\pm \pi$, i.e. in the range of values of interest for polarization conversion, the maximum change of the insertion loss is $2.3 \mathrm{~dB}$. If the power of the input signal is high enough, the Brillouin process saturates and appreciable changes on the insertion loss of the polarization controller can be seen. 


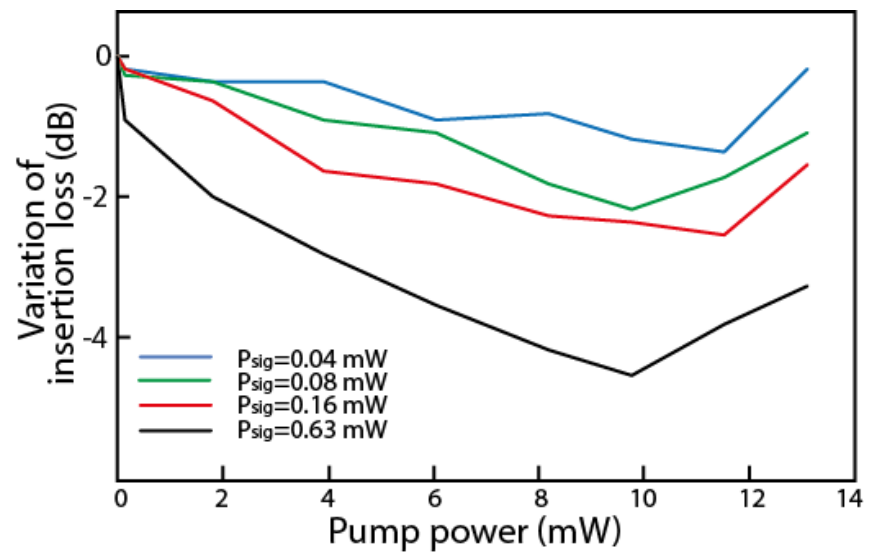

Figure 3.9 Variation of the insertion loss of the polarization controller as a function of pump power for signals with different input power at the HLNF.

As in other applications of SBS, the bandwidth of the Brillouin response can be tailored. Since Brillouin response is the convolution of the natural Brillouin gain spectrum with the normalized pump power spectrum, arbitrary polarization responses can be achieved by engineering the pump spectrum. Figure 3.10 shows the frequency dependence of the retardance induced by the Brillouin natural response (blue) and a flat-top retardance obtained with a multi-tone pump (red), which shows a maximum ripple of $2^{\circ}$.

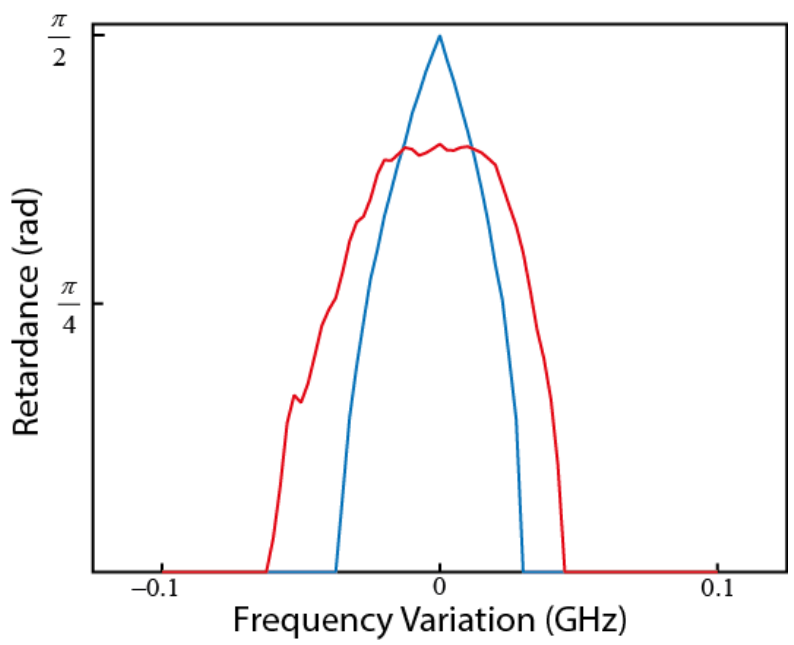

Figure 3.10 Control of the retardance bandwidth. (a) Single pump configuration. (b) Multi-tone pump configuration. 
Another interesting feature of the presented nonlinear polarization controller is that, unlike most Brillouin processing schemes, it introduces little additional noise due to the lack of net gain. To assess the noise addition by the polarization controller, the noise figure (NF) is defined as the ratio of SNR of the optical signal when the controller is off and on. An electrical method to measure NF has been employed since it provides a more complete measure of the intensity noise generated by the controller [83]. Noise has been measured using the setup shown in Figure 3.11 where an RF signal (2.5 GHz) has been used to modulate the optical signal. After heterodyne detection, the SNR of the beat note has been measured with an electrical spectrum analyzer.

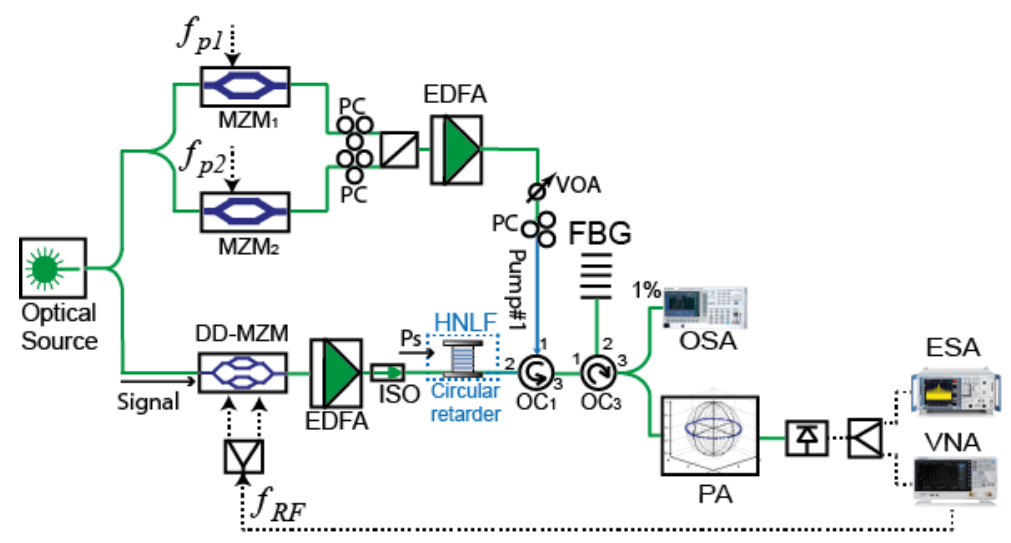

Figure 3.11 Block diagram of a nonlinear all-optical polarization controller used to measure noise. HLNF: Highly nonlinear fiber. OC: Optical circulator; ISO: Isolator. VOA: Variable Optical Attenuator. FBG: Fiber Bragg grating. OSA: Optical spectrum analyzer. PA: Polarization analyzer. ESA: electrical spectrum analyzer. VNA: vector network analyzer.

The Brillouin-induced noise $\left(S_{P}(f)\right)$ must be separated from the total measured noise $\left(\left.S_{E S A}(f)\right|_{\text {mea }}\right)$ which also includes receiver thermal noise $\left(\left.S_{E S A}(f)\right|_{t h}\right)$, detected shot noise $\left(2 q i_{m e a}\right)$ and the frequency response of the system [83]. The thermal noise correction has been performed from measurement of the thermal noise density with the input light blocked. The correction of the system transfer function has been performed using a RIN transfer calibration setup. Finally, the shot noise has been corrected from measurements of the optical and electrical spectral of the amplitude modulated optical signal and its associated microwave beat note, respectively.

The intensity noise density produced by the polarization controller $\left(S_{p}(f)\right)$ has been derived from [83]: 


$$
S_{P}(f)=\frac{\left.S_{E S A}(f)\right|_{\text {mea }}-\left.S_{E S A}(f)\right|_{t h}}{\frac{\left.S_{E S A}(f)\right|_{c a l}}{R I N_{c a l}(f) P_{c a l}^{2}}}-\frac{2 q i_{\text {mea }}}{\mathfrak{R}^{2}}\left[\frac{W^{2}}{H z}\right]
$$

Then, the noise figure is [83]

$$
N F=\frac{P_{S} S_{P}(f)+2<G P_{s}>h v}{2 h v<G P_{S}>^{2}}
$$

where the gain $\left(G^{2}\right)$ has been derived from spectral analysis of the optical receiver photocurrent and $P_{s}$ is the power at the fiber input.

The change of the measured NF for a rotation between linear at 0 and $2 \pi$ has been measured showing a maximum variation of $1.5 \mathrm{~dB}$. For the sake of comparison, the proposed technique has been compared to polarization pulling, which induces a maximum pulling of $\pi / 2$. Given the gain induced by polarization pulling, the signal power for polarization pulling has been modified to produce the same RF beat note power. As it can be seen in Figure 3.12, the polarization rotator experiences a NF variation of less than $1 \mathrm{~dB}$ with polarization pulling showing almost a $5 \mathrm{~dB}$ increment.

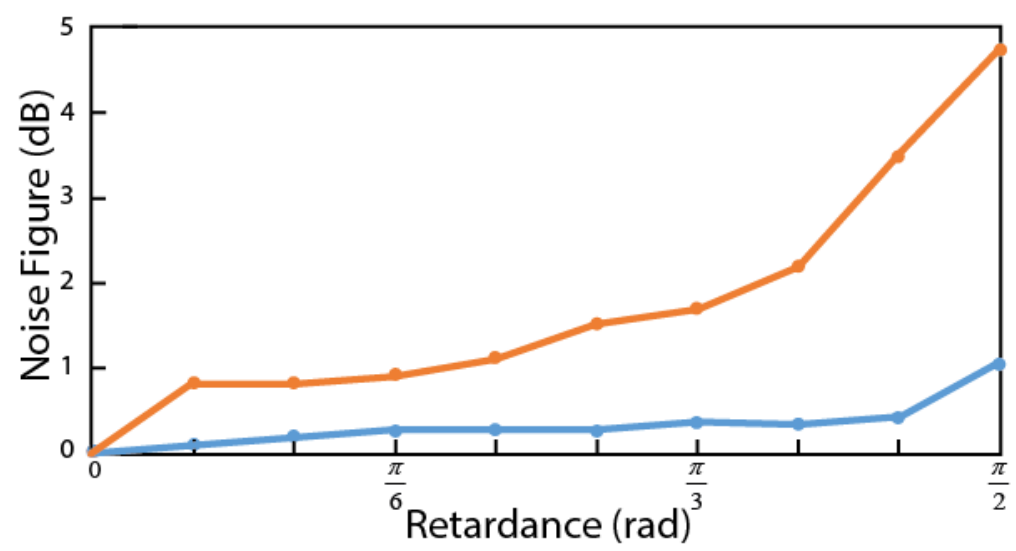

Figure 3.12 Comparison of the change of the Noise Figure for the proposed method (blue) and polarization pulling (orange).

Finally, stability of the SOP has been measured. Figure 3.13(a) show the temporal evolution of the Stokes vector components for a signal with input power at the HLNF of $0.16 \mathrm{~mW}$ and linear polarization at $45^{\circ}$ that is rotated with the Brillouin polarization controller to a linear signal at $135^{\circ}$, as depicted in Figure 3.13(b). 
(a)

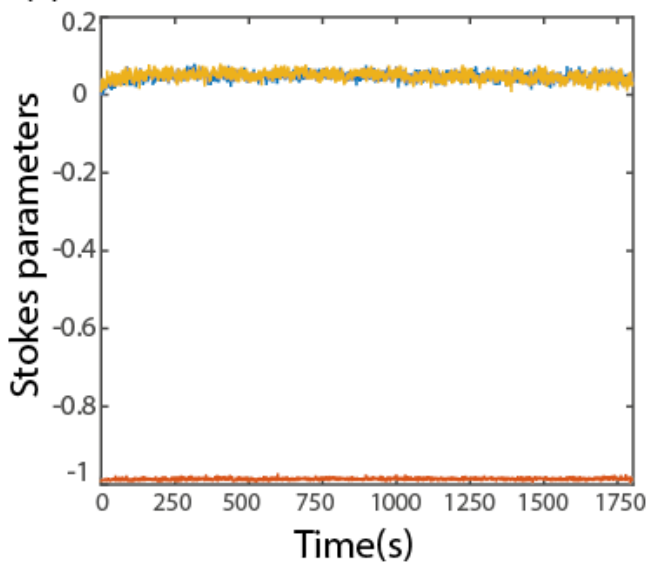

(b)

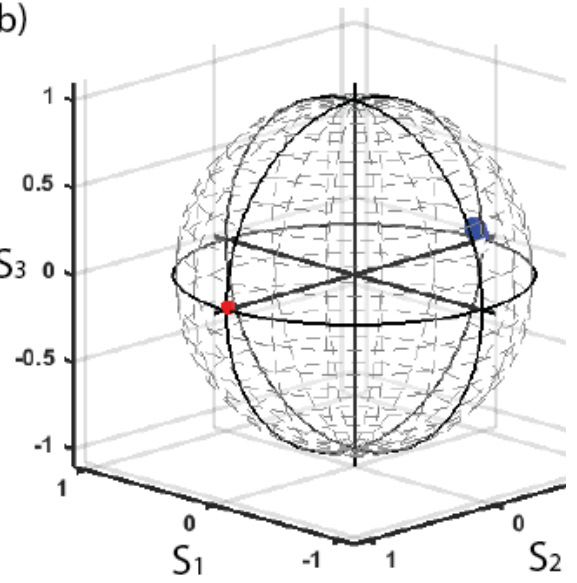

Figure 3.13 Temporal stability of the signal SOP at the output of the polarization controller. (a) Stokes parameters of the signal output with linear SOP at $135^{\circ}$ as a function of time: (Color online) S1 (blue solid curve), S2 (red solid curve) and S3 (yellow solid curve). Stokes parameters are normalized to S0. (b) (Red) SOP of the signal without SBS, (blue) Signal SOP with SBS polarization conversion

The standard deviation of the Stokes parameters of the signal polarization with and without SBS polarization conversion is shown in Table 3.1.

Table 3.1 Standard deviation of Stokes parameters from Figure 3.13(a)

\begin{tabular}{|l|c|c|c|}
\hline & S1 & S2 & S3 \\
\hline $\begin{array}{l}\text { Rotated signal } \\
\text { SOP }\end{array}$ & $8.3 \times 10^{-3}$ & $3.0 \times 10^{-3}$ & $9.9 \times 10^{-3}$ \\
\hline $\begin{array}{l}\text { Unrotated signal } \\
\text { SOP }\end{array}$ & $1.3 \times 10^{-3}$ & $0.6 \times 10^{-3}$ & $1.1 \times 10^{-3}$ \\
\hline
\end{tabular}

\subsection{Conclusion}

In this section, a new all-optical functionality, dynamic polarization rotation, has been introduced. Using stimulated Brillouin scattering, light polarization can be actively tailored, independently of adjacent signals. Experiments have shown that the full Poincaré sphere can be reached while introducing optical power changes smaller than $2.5 \mathrm{~dB}$. Since Brillouin processing has the lowest power threshold among nonlinear effects in 
silica optical fibers, this effect can be easily harnessed. The technique uses a combination of gain and loss Brillouin responses that avoids the generation of spontaneous Brillouin noise, which conventionally is the main limitation of Brillouin optical processing. This new functionality combined with its low noise opens new opportunities in polarization control from the synthesis of light with complex frequency-dependent polarization structures for chemistry, imaging and optical communications as well as a new approach to revisit some applications where Brillouin processing has been constrained by its noisy nature. 


\section{Chapter 4}

\section{SBS-based Photonic Microwave Filtering}

\subsection{Photonic Microwave Filtering}

Among the different areas of microwave photonics, filtering has attracted considerable attention [84]. Filtering is one of the main functions performed in communication systems, radar and sensing instruments. Although typically performed using electronic circuitry, photonics allows for an alternative implementation [84].

Photonic microwave filtering offers several features that are not easily achievable in the microwave/digital domains such as wide bandwidth and tuning range, reconfigurability of the frequency response [57] and the ability of some architectures to be seamlessly combined with radio over fiber distribution links.

Extensive research has been carried out and different photonic microwave filter architectures have been presented, including transversal filters [57], [85], comb sources [86] or fiber Bragg gratings [87].

With the aim of advancing towards practical implementations of photonic microwave filters able to commercially compete with pure electronic approaches, two main paths are being followed. One relies on photonic integration [49]. Some schemes (e.g. [58], [88]-[92]) can be integrated in a Photonic Integrated Circuit (PIC) to implement photonic analogues of discrete microwave filter devices. Integration offers compact low-cost solutions with the potential to avoid the performance degradation in terms of Q-factor suffered by conventional microwave filters when miniaturized. 
A second path is based on exploiting nonlinear effects such as Four-Wave Mixing [93] to develop efficient architectures for photonic microwave filters. Among nonlinear effects, stimulated Brillouin scattering (SBS) is especially well suited to filter microwave signals [89], [90], [88], [91], [94], [95], [96]. SBS-based schemes are very stable, can exhibit a single bandpass that can be tuned and reconfigured to show flat-top responses and they can easily implement narrow filter responses in the range of $\mathrm{MHz}$ to $\mathrm{GHz}$, as opposed to the periodic and rather broadband responses showed by transversal filters. In addition, SBS has integration potential (e.g. [96]), and therefore it can be used to leverage the benefits provided by both approaches.

\subsection{Review of SBS-based Photonic Microwave Filtering}

As previously described, SBS is very appealing for microwave filtering because it can be used to implement narrow filter responses with tuning and reconfiguration capabilities. In addition, this nonlinear effect can be stimulated in integrated circuits which is critical for cost reduction [96].

The basic scheme for a SBS photonic microwave filter [89] is based on the amplitude modulation of the signal to be filtered with a pump that is derived from the same optical source through external modulation to achieve a very stable frequency response. A filter response with two passbands is obtained following this approach. Filters implemented through this approach can be continuously tuned over a wide bandwidth and the filter response can be dynamically reconfigured. A pure single passband filter can also be achieved if the pump is created with a parallel external modulator to implement a single sideband modulation without carrier.

The rejection ratio of the filter can be enhanced if the RF signal to be filtered is phase modulated and SBS is used to break the symmetry between both sidebands. In particular, when the SBS gain/loss response falls into only one sideband of the RF modulated optical, it selectivity breaks the phase balance between sidebands. After O/E conversion, the SBS response is indirectly transferred into a single bandpass filter response. Using this technique, in [97] a filter response with an out-of-band rejection of $55 \mathrm{~dB}$ and a full width at half-maximum bandwidth of $16 \mathrm{MHz}$ was obtained.

Another area of interest has been the engineering of the filter response. Since it is given by the convolution between the natural SBS response and the pump, different filter responses can be implemented through the control of the pump signal [89]. This allows the implementation of flat-top filter responses with good shape factor by increasing the SBS gain using two stages in cascade to avoid pump depletion [90]. It is done by increasing the filter bandwidth since the skirt selectivity of SBS-based photonic microwave filters is given by the slow-decaying Lorentzian tail of the natural Brillouin response, which produces filters with poor rejection of close adjacent channels. Flat-top responses can be obtained using a multi frequency electrical comb [95] or a single microwave tone can be 
swept to for the swept [91], [98]. In the latter, there is only a single frequency at any specific time, which avoids nonlinearities inducing gain ripple and enhances the out of band rejection. The flat-top response can be further optimized using a digital feedback control technique on the Brillouin pump. Using this approach, a filter response with a $\mathrm{GHz}$ bandwidth and a shape factor of 1.056 was reported [98].

Another approach to enhance out-of-band rejection of bandpass filters based on polarization modulation have been presented. This modulation scheme provides upper and lower sidebands with the same amplitude and a $180^{\circ}$ phase difference, i.e. the upper and lower sidebands are polarized orthogonally regard to the optical carrier. This orthogonality guarantees high out-of-band rejection. The passband of the filter is selected by polarization pulling effect using one or two stages of SBS in an optical fiber. The bandpass of the filter response obtained through polarization modulation, polarization pulling and two stages achieved a record out-of-band rejection of $80 \mathrm{~dB}$ with a bandwidth of 7.7 $\mathrm{MHz}$ [99].

SBS can also be used to implement notch filters. Photonic microwave notch filters based on SBS are capable of frequency tuning and bandwidth reconfiguration. For example, a photonic microwave notch filter that exhibits a notch with $>40 \mathrm{~dB}$ has been presented [100]. A different implementation is based on the RF cancellation technique which provides notch responses with a rejection of $60 \mathrm{~dB}$ [101].

SBS on integrated platforms has been researched by the group of Prof. Eggleton in Australia. In particular, they researched photonic microwave filters relying on an integrated rib waveguide of chalcogenide $\mathrm{AS}_{2} \mathrm{~S} 3$. The performance of this architecture attained less suppression $(20 \mathrm{~dB})$ and required higher powers $(350 \mathrm{~mW})$ [102]. Following the technique presented in [101], a notch response with high suppression ( $>50 \mathrm{~dB})$ was achieved despite the fact that little SBS gain was obtained (just $1 \mathrm{~dB}$ ) [96]. Later, this same technique has been implemented in suspended silicon nanowires implementing notches with a suppression of $48 \mathrm{~dB}$ [103].

Despite these works, filter responses obtained from SBS are still not able to fulfill the requirements for filter masks of real applications. One of the reasons behind this fact is that the filter slope is not high enough. In this chapter, new methods to enhance the filter slope of SBS-based photonic microwave filters are researched. Additionally, a new technique based on the work carried out in Chapter 3 is proposed to obtain notch filters with very high suppression of unwanted signals. 


\subsection{Photonic microwave filter with steep skirt selectivity based on stim- ulated Brillouin scattering}

\subsubsection{Introduction}

The principle of operation is based on combining a SBS gain response with two frequency-offset SBS loss responses that are used to attenuate the slowly decaying tail of the Lorentzian response. When this engineered SBS response is applied to a phase-modulated microwave signal, SBS introduces a gain/loss over the sidebands that breaks the out-of-phase condition between sidebands. Thus, any microwave signal going through the system is eliminated but in the band where the combined SBS response has been applied. The combination of gain and loss responses in SBS has been previously used to reduce the optical Brillouin bandwidth [104] at the cost of severely reducing its gain. Instead, here it is shown both theoretically and experimentally that for a SBS-based microwave filter the slope can be enhanced while keeping the rejection ratio by using phase modulation jointly with a combination of gain and loss responses.

\subsubsection{Photonic microwave filter architecture}

Figure 4.1 describes the principle of operation. By means of SBS, a pump counterpropagating the signal induces both a gain and a loss response in amplitude that are up- and down-shifted in frequency $\left(f_{B}\right)$, respectively [1]. Figure 4.1(a) (left) shows how the gain is combined with two loss (notch) responses in amplitude. These notches introduce a sharp drop in the natural Lorentzian tail (dashed blue) as it can be seen in the combined response (black solid) at the cost of a slight reduction of the combined gain. The same happens in the other sideband (Figure 4.1(a) right).

To obtain the combined SBS response, three pumps counterpropagating the phase-modulated microwave signal are needed. They are generated using a double-sideband suppressed-carrier (DSB-SC) modulation as shown in Figure 4.1(b). In the lower band, the three pumps are labelled $f_{c}-f_{1}, f_{c}-f_{2}, f_{c}-f_{3}$, where $f_{c}-f_{1}$ induces a Stokes gain wave $P_{g 1}\left(f_{c}-f_{R F}\right)$ (that generates the central SBS gain, $g_{1}\left(f_{c}-f\right)$ ) and the other two pumps: $f_{c}-f_{2}, f_{c}-f_{3}$ induce two Stokes loss waves $P_{\alpha 2}\left(f_{c}-f_{R F}\right), P_{\alpha 3}\left(f_{c}-f_{R F}\right)$ in the lower band signal (that induce SBS loss, $\alpha_{p}\left(f_{c}-f\right)$, at the sides of the central SBS gain). Whereas for the upper band, $f_{c}+f_{1}$ induce a Stokes loss wave $P_{\alpha 1}\left(f_{c}+f_{R F}\right)$ and $f_{c}+f_{2}, f_{c}+f_{3}$ induce two Stokes gain waves $P_{g 2}\left(f_{c}+f_{R F}\right), P_{g 3}\left(f_{c}+f_{R F}\right)$. By changing the frequency of the local oscillators used to generate the pumps, the filter response can be tuned. 


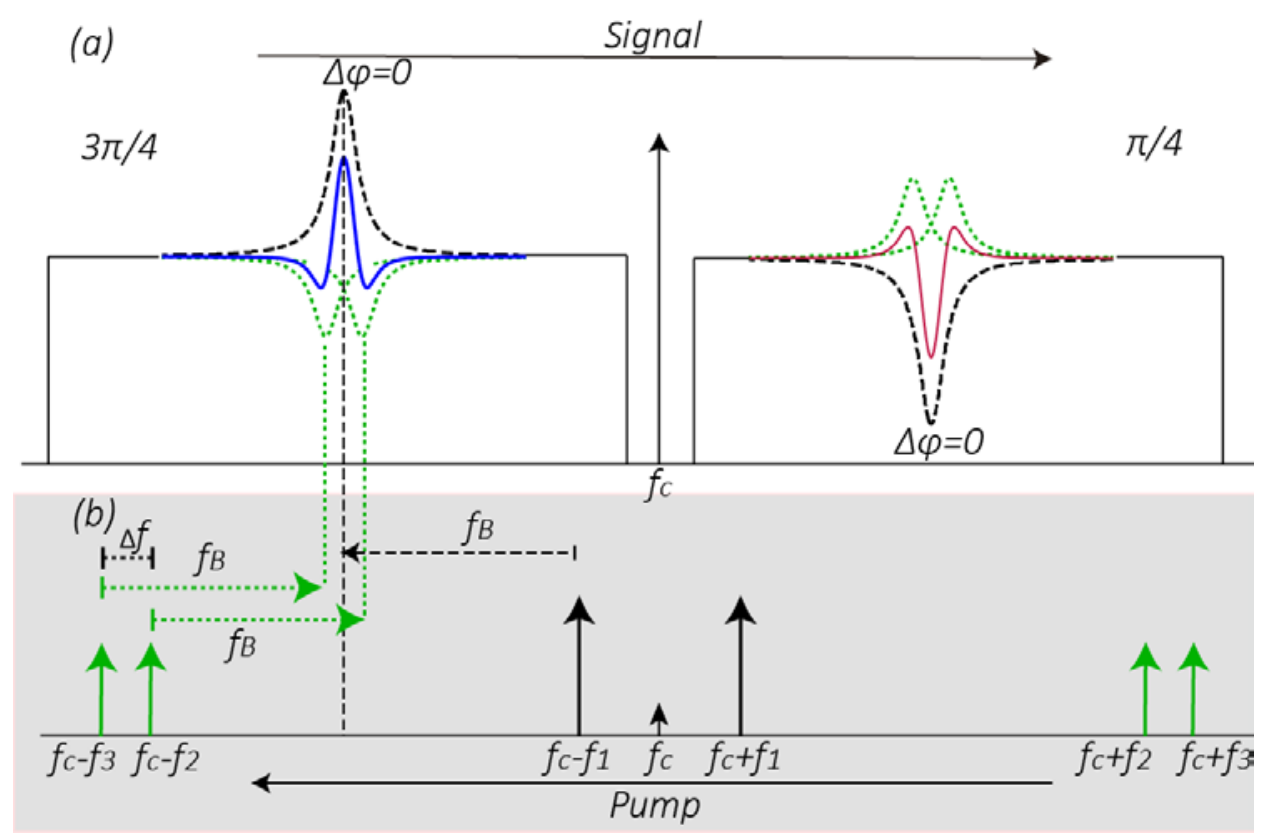

Figure 4.1 (Color online) Principle of operation. (a) Optical spectrum of the forward signal with SBS amplitude gain and loss responses. (b) Backward pump signals with frequency $f_{\mathrm{P}}$, where $\mathrm{P}=1$, 2, 3.

A theoretical model of the SBS-driven filter response can be obtained. For the sake of simplicity, the case of a single pump is considered first. For this case, both the SBS gain and loss was described in the Equation 2.7 and Equation 2.8.

To estimate the SBS gain and loss, the coupled differential equations that define SBS in steady state have to be solved [1]. Here, considering the solution proposed in [18], the spatial distribution of the field intensity is: $I_{P}=P_{P} / A_{\text {eff }}$, it is assumed that the losses in the fiber are usually low. Assuming pump depletion, the Stokes gain $\left(P_{g P}\left(f_{c} \pm f_{R F}\right)\right)$ and loss $\left(P_{\alpha P}\left(f_{c} \mp f_{R F}\right)\right)$ waves were found from Equation 2.25.

The filter frequency response with a single SBS pump can be derived using the small signal approximation and omitting the DC component and higher-order harmonics. It is given by [94], 


$$
\begin{aligned}
\left|H\left(f_{R F}\right)\right|^{2}= & \frac{P_{R F o u t}}{P_{R F i n}} \\
= & \frac{\pi^{2} \Re^{2} Z_{\text {in }} Z_{0} P_{0}^{2} \alpha_{0}^{2}}{4 V_{\pi}^{2}}\left[\left|P_{g P}\left(f_{c}-f_{R F}\right)\right|^{2}+\left|P_{\alpha P}\left(f_{c}+f_{R F}\right)\right|^{2} \quad \text { Equation } 4.1\right. \\
& -P_{g P}\left(f_{c}-f_{R F}\right) P_{\alpha P}\left(f_{c}+f_{R F}\right)-P_{g P}\left(f_{c}-f_{R F}\right)^{*} \\
& \left.P_{\alpha P}\left(f_{c}+f_{R F}\right)^{*}\right]
\end{aligned}
$$

where $P_{R F o u t}$ is the RF power at the output of PD, $P_{R F i n}$ is the RF power at the input as shown in Figure 4.3, $\Re$ is the PD responsivity, $Z_{0}$ is the load resistance, $P_{0}$ is the optical power of the light, $Z_{\text {in }}$ is the input impedance of the DD-MZM, $\alpha_{0}$ is the optical loss between the laser output and the PD input.

When additional pumps are present, the filter response can be obtained from Equation 4.1. In particular, for the case of three Stokes gain/loss waves, the filter response can be described by Equation 4.2 when phase modulation is used taking into account that, in the lower sideband of the signal, there are three interacting signals $P_{g 1}\left(f_{c}-f_{R F}\right), P_{\alpha 2}\left(f_{c}-\right.$ $\left.f_{R F}\right), P_{\alpha 3}\left(f_{c}-f_{R F}\right)$, whereas in the upper sideband of the signal the interaction is produced by another three terms: $P_{\alpha 1}\left(f_{c}+f_{R F}\right), P_{g 2}\left(f_{c}+f_{R F}\right), P_{g 3}\left(f_{c}+f_{R F}\right)$.

$$
\begin{aligned}
\left|H\left(f_{R F}\right)\right|^{2}= & \frac{\pi^{2} \Re^{2} Z_{i n} Z_{0} P_{0}^{2} \alpha_{0}^{2}}{4 V_{\pi}^{2}} \\
& \cdot\left[\left|P_{g 1}\left(f_{c}-f_{R F}\right)\right|^{2}\left|P_{\alpha 2}\left(f_{c}-f_{R F}\right)\right|^{2}\left|P_{\alpha 3}\left(f_{c}-f_{R F}\right)\right|^{2}\right. \\
& +\left|P_{\alpha 1}\left(f_{c}+f_{R F}\right)\right|^{2}\left|P_{g 2}\left(f_{c}+f_{R F}\right)\right|^{2}\left|P_{g 3}\left(f_{c}+f_{R F}\right)\right|^{2} \\
& -P_{g 1}\left(f_{c}-f_{R F}\right) P_{\alpha 2}\left(f_{c}-f_{R F}\right) P_{\alpha 3}\left(f_{c}-f_{R F}\right) \\
& . P_{\alpha 1}\left(f_{c}+f_{R F}\right) P_{g 2}\left(f_{c}+f_{R F}\right) P_{g 3}\left(f_{c}+f_{R F}\right) \\
& -P_{g 1}\left(f_{c}-f_{R F}\right)^{*} P_{\alpha 2}\left(f_{c}-f_{R F}\right)^{*} P_{\alpha 3}\left(f_{c}-f_{R F}\right)^{*} \\
& . P_{\alpha 1}\left(f_{c}+f_{R F}\right)^{*} P_{g 2}\left(f_{c}+f_{R F}\right)^{*} P_{g 3}\left(f_{c}+f_{R F}\right)^{*}
\end{aligned}
$$

Unlike single sideband amplitude modulation, given by the first term of Equation 4.2, phase modulation results in the subtraction of several terms that lead to partial cancellation of the long decaying tails of Brillouin response. Figure 4.2 shows this effect. The figure shows the total theoretical filter response (black solid line) for the interaction of three gain/loss waves with a phase modulated signal, as given by Equation 4.2 as well as the contribution of each term of the equation. The first term of Equation 4.2 (blue dotted in Figure 4.2) corresponds with the gain response obtained from the combination of the Brillouin gain with two notches. This gain profile is applied on the lower sideband of the 
signal and shows an enhanced slope in comparison to the natural gain Lorentzian response of SBS (as it can be seen in Figure 4.1, blue solid). This first term (blue dotted) corresponds to single sideband modulation, as in [104].

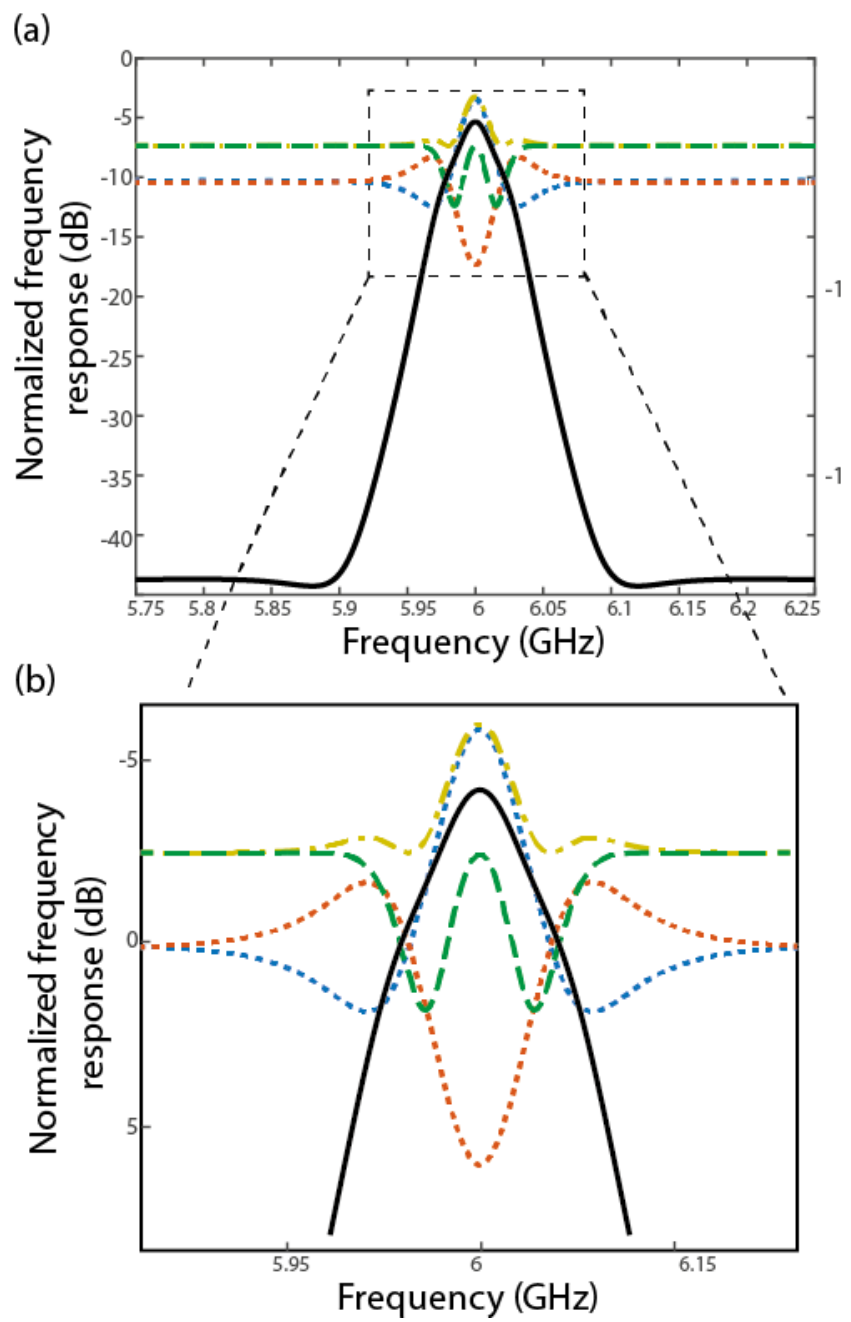

Figure 4.2 (Color online) (a) Evaluation of the terms of Equation 4.2. (black solid) Normalized total frequency response of the SBS-based filter obtained from Equation 4.2. (blue dotted) Amplitude frequency response of the gain SBS response (lower sideband), i.e. first term of Equation 4.2. (red dotted) Amplitude frequency response of the loss SBS response (upper sideband), i.e. second term of Equation 4.2. (yellow dot-dashed) Combination of the first and second terms of Equation 4.2. (green dashed) Third and fourth term of Equation 4.2. (b) Detail of Figure 4.2 (a). 
On the other hand, the second term (red dotted in Figure 4.2) corresponds to the loss response obtained from the combination of the natural Brillouin loss with two gain profiles at the sides, which can also be seen in the upper sideband of Figure 4.1 (red solid). These two modified SBS responses are generated in the optical domain. However, after photodetection, a third and a fourth terms appear (their sum is shown in green dashed in Figure 4.2) which depend on the phase information of all Stokes waves. Equation 4.2 shows that the first and second terms are added together (Stokes gain + Stokes loss) (showed as the yellow dot-dashed line in Figure 4.2) whereas the third and fourth term have the opposite sign. This latter two terms introduce a small reduction on the gain of the filter passband but, on the positive side, they cancel the Lorentzian tail and improve the filter selectivity.

Key design parameters are the values of $f_{2}$ and $f_{3}$ which have to be chosen to optimize the slope of the slow-decaying Lorentzian tail of the gain response while minimizing the reduction of the gain of the passband. In amplitude modulation, the loss responses induce a reduction on both bandwidth and gain with no enhancement on selectivity. Unlike this, phase modulation induces a smaller change of bandwidth but with a steeper slope. The tunability of the central frequency of the filter response is bounded by the bandwidth of the electrooptic components and the tuning range of the three local oscillators.

\subsubsection{Experimental results}

Figure 4.3 shows the experimental setup used to validate the concept. The light from a DFB source at $1548 \mathrm{~nm}$ with an ouput power of $10 \mathrm{dBm}$, was divided by a directional coupler 50/50. The upper path of the setup was used to generate the pump while the lower one corresponds to the signal. In the upper path, the light entered a $40 \mathrm{GHz}$ intensity modulator (MZM) biased at minimum transmission (MITB), generating a DSB-SC modulation. The pump signal $\mathrm{f}_{\mathrm{p}}^{\prime}$, had three components, which induce one gain and two loss responses over the lower sideband and the opposite $f_{p}$ for the upper sideband. The pump waves were generated using a vector signal generator (Agilent ESG E4438C) and a signal generator (Rohde\&Schwarz SMR 20). The particular frequencies and amplitudes of these signals were obtained from simulations performed with the model described in Section 2. Optical amplification ( $40 \mathrm{~dB}$ ) was used to boost the pump signal and, finally, an optical circulator directed the pump signal to the nonlinear medium (a reel of $1 \mathrm{~km}$-long HNLF with Brillouin linewidth $\gamma_{B}=40 \mathrm{MHz}$ and Brillouin frequency shift $f_{B}=9.64 \mathrm{GHz}$ ). In the lower path, the data signal was phase modulated using a 40 $\mathrm{GHz}$ dual-drive optical modulator (DD-MZM) and a 2-18 GHz hybrid $90^{\circ}$ coupler ([94]; [105]). The DD-MZM was biased at $9.7 \mathrm{~V}$ (maximum transmission point, MATB) The DC half-wave voltage of the DD-MZM was measured to be $5.4 \mathrm{~V}$.

Unlike [94] where it was used to dynamically switch between bandpass and notch filter responses, we just used a DD-MZM for phase modulation due to the unavailability of a phase modulator in our laboratory. The modulated signal passed back through the HNLF 
and reached a PIN photodiode with a bandwidth from $30 \mathrm{KHz}$ to $9 \mathrm{GHz}$. Finally, the frequency response of the filter was measured with a vector network analyser (VNA, HP 8510C).

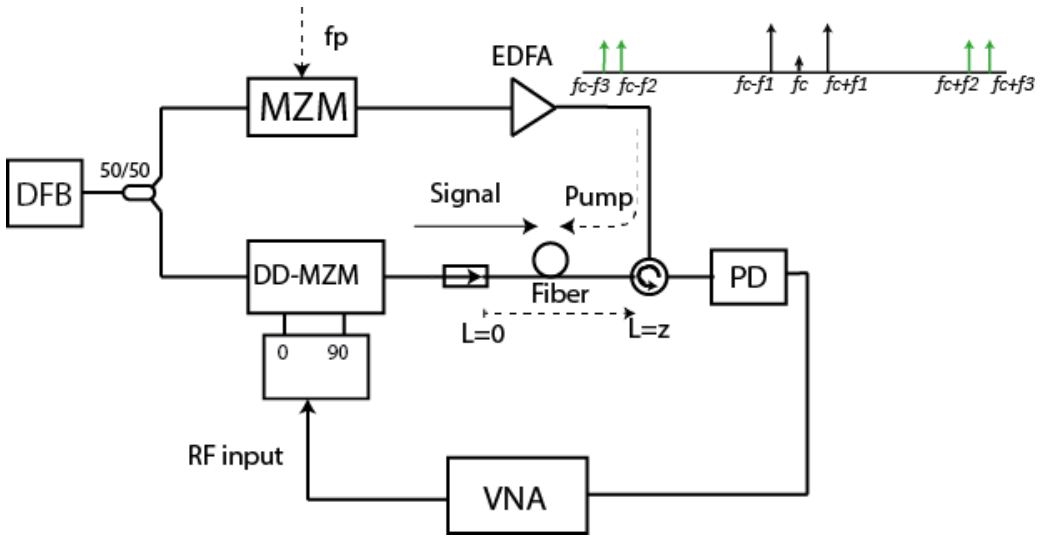

Figure 4.3 Block diagram of the experimental setup. DFB: distributed feedback laser, MZM:

Mach-Zehnder modulator, DD-MZM: Dual-Drive MZM, VNA: vector network analyzer.

The microwave filter response was measured as shown in Figure 4.4(a) (black solid). The experimental result showed good agreement with the theoretical model Equation 4.2. For the sake of comparison, the filter responses obtained directly applying a single pump [89] have been included for two cases with the same pump power at $f_{1}$ : in the first case, using $f_{c}-f_{1}, f_{c}+f_{1}$ the SBS gain reaches $15 \mathrm{~dB}$ and in the second case, applying the proposed method, SBS gain reaches $5 \mathrm{~dB}$, where the gain was measured when the modulator signal DD-MZM acted as a lower side band carrier with LSB+C $\left(V_{\text {bias }}=\right.$ $3 \pi / 2$ ). From the comparison shown in Figure 4.4(a) it can be seen that in both cases (SBS natural gain of $15 \mathrm{~dB}$ and $5 \mathrm{~dB}$ ) the selectivity is improved. The tradeoff between the filter gain, bandwidth and slope can be modified by changing the frequencies of the pumps as well as their amplitudes. Figure 4.4(b) show the tuning capability of the filter by changing the frequency of the microwave oscillators. 
(a)
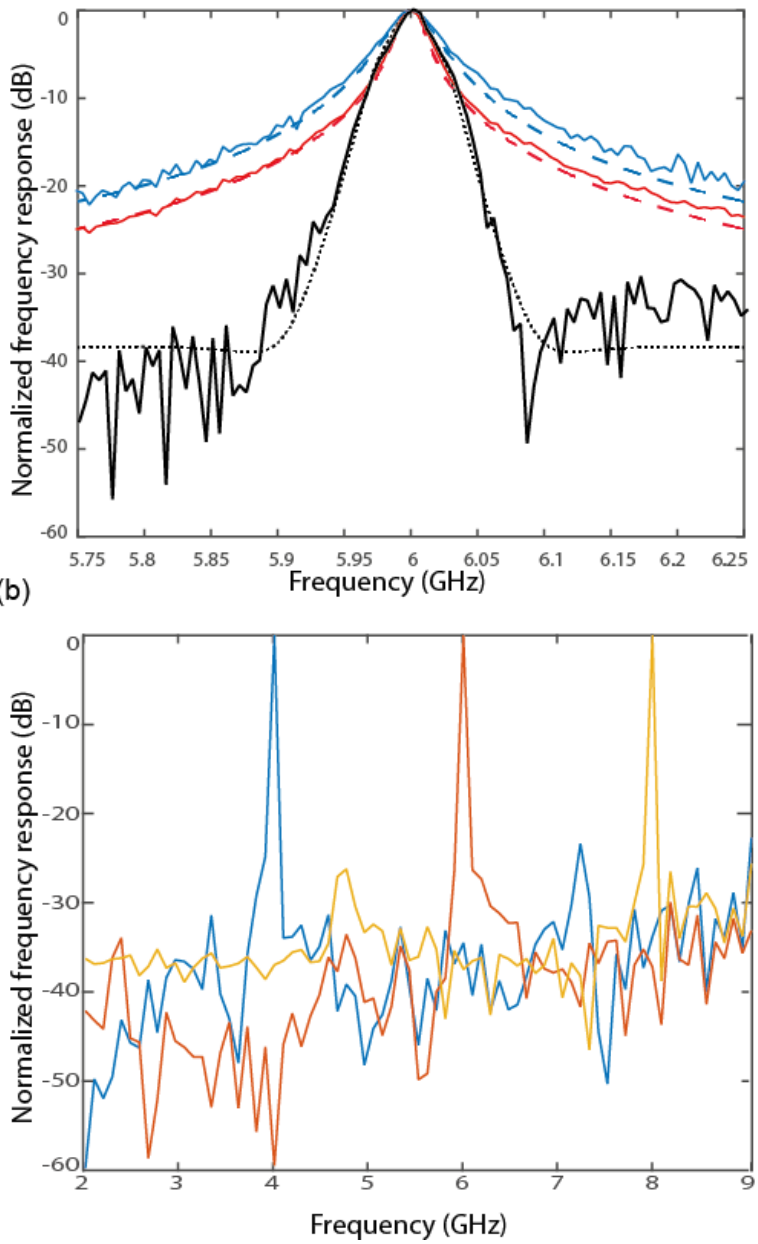

Figure 4.4 (a) (Color online) (Black solid) Normalized RF frequency response of the SBS-based filter; (black dashed) theoretical filter response obtained from Equation 4.2 with $f_{1}=15.64 \mathrm{GHz}$, $f_{2}=3.62 \mathrm{GHz}, f_{3}=3.66 \mathrm{GHz}$; (blue dashed) theoretical filter response with a single pump (Equation 4.1) and natural gain of $5 \mathrm{~dB}$; (blue solid) experimental filter response with a single pump and natural gain of $5 \mathrm{~dB}$ with $f_{1}=15.64 \mathrm{GHz}$; (red dashed) theoretical filter response with a single pump (Equation 4.1) and SBS natural gain of $15 \mathrm{~dB}$; (red solid) experimental filter response with a single pump and natural gain of $5 \mathrm{~dB}$ with $f_{1}=15.64 \mathrm{GHz}$. (b) Normalized frequency response of the SBS-based tunable filter.

By mapping the bandpass filter response to a lowpass function, the slope response can be estimated (Figure 4.5). The figure shows a 3-fold enhancement of the slope, reaching 
$16.7 \mathrm{~dB} /$ oct in comparison to the $6 \mathrm{~dB} /$ oct from single pump responses (single pole filter response) [106]. A third-order filter has been implemented as corresponds to the combination of a bandpass and two notch responses in cascade [107].

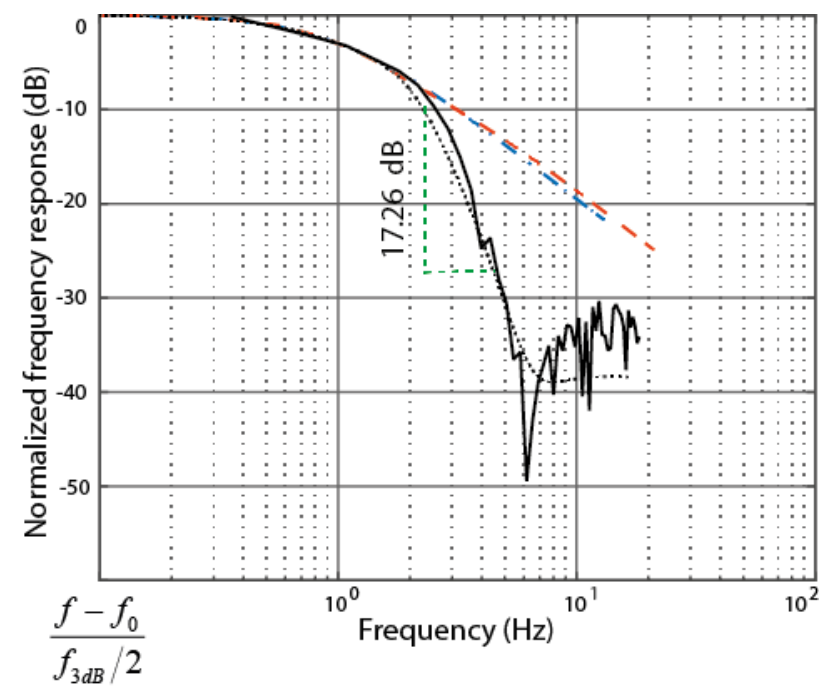

Figure 4.5 Bode diagram of the SBS-based filter response. Experimental filter response (black solid) with $16.7 \mathrm{~dB} /$ oct; theoretical filter response obtained from equation Equation 4.2 (black dotted) with $17.26 \mathrm{~dB} /$ oct; theoretical response with a single pump and $5 \mathrm{~dB}$ gain (blue dash-dotted) with $3.79 \mathrm{~dB} /$ oct; theoretical response with a single pump and $15 \mathrm{~dB}$ gain (red dashed) with 4.08 dB/oct.

Additionally, a filter with a flat-top response has been obtained by sweeping the pump frequency and including a feedback algorithm [95] to control the sweeping gain pump. The experimental response is shown in Figure 4.6. The 3dB bandwidth is $84 \mathrm{MHz}$ with a slope of around $18 \mathrm{~dB} /$ oct. Since the technique only produce an effect on the tails of the response, it does not introduce any particular limitation to the filter bandwidth.

Finally, the noise degradation introduced by SBS filtering was analyzed. Measurements show that the signal to noise ratio is degraded in $5 \mathrm{~dB}$ when the SBS-filter is used. 


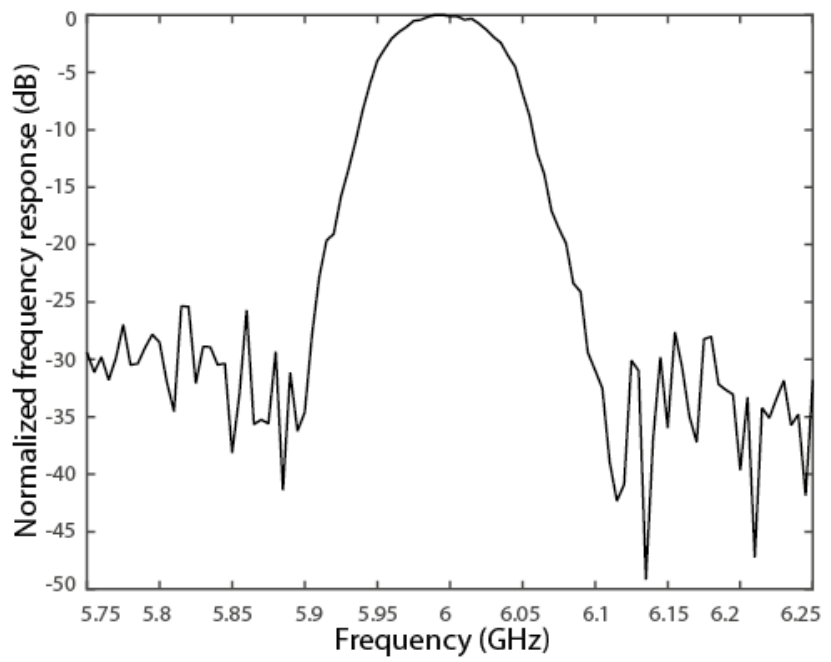

Figure 4.6 Normalized RF frequency response of the SBS-based filter with $84 \mathrm{MHz}$ FWHM bandwidth.

\subsubsection{Conclusions}

In this section has been shown both through a theoretical model and experiments that phase modulation induces a cancellation on the Lorentzian tails when a combination of gain and loss Brillouin responses are applied to implement a SBS-based photonic microwave filter. This results in enhanced selectivity of the filter response. The method does not require high gain and therefore the noise figure of the link is barely affected. At the same time, due to this fact, the approach is suitable to be exploited in Photonic Integrated Circuits (PIC) for microwave processing [90] since given their short interaction length little gain can be obtained. Further work is needed to further enhance selectivity in order to match filter specification masks and pave the way towards integrated photonic microwave filters competitive with standard microwave implementations.

\subsection{Brillouin Microwave Filter with enhanced Skirt Selectivity using a Birefringent Fiber}

\subsubsection{Introduction}

An alternative approach for improving skirt selectivity while keeping easy tunability is explored in this section. It relies on photonic phenomena to simplify the architecture. In particular, it uses the usually deleterious effect of the double Brillouin gain/loss response happening in low birefringence optical fibers [17]. 


\subsubsection{Principle of Operation}

Considering a birefringent fiber and assuming operation in the undepleted pump regimen, the SBS amplification can be seen as composed of two SBS gain terms [17]: maximum gain $\left(G_{\max }\right)$ and minimum gain $\left(G_{\min }\right)$, with orthogonal states of polarization (SOP). The SOP of the maximum gain is parallel to the conjugate of the pump wave $\left(\overline{E_{p}^{*}}\right)$, whilst the SOP of the minimum gain is orthogonal to the conjugate of the pump wave, as shown in Figure 4.7 (a). Similarly, SBS attenuation has two terms: maximum loss $\left(A_{\max }\right)$ and minimum loss $\left(A_{\min }\right)$.

If the SOP of a phase-modulated optical carrier is aligned at $45^{\circ}$ in relation to the conjugate of the pump wave, the signal components on each of the orthogonal axis will experience maximum and minimum SBS gain/loss respectively, as shown in Figure 4.7 (a) for the case of SBS gain.

Thus, each orthogonal polarization carries a copy of the same microwave-modulated optical signal but with a different SBS gain. It is possible to split the signals $E_{y} G_{\max }$ and $E_{x} G_{\min }$ using a polarization beam splitter. If the relative amplitude between these two responses $\left(E_{y} G_{\max }\right.$ and $\left.E_{x} G_{\min }\right)$ is adjusted, they can be subtracted using a balanced photodiode to suppress the tails of the SBS gain, as shown in Figure 4.7(b). Thus, a considerable reduction of the decaying tails can be obtained while having a small effect on the passband loss.

\subsubsection{Theory}

Assuming no pump depletion and that the losses in the fiber are low, the SBS gain and loss induced by a counterpropagating pump was described in the Equation 2.7 and Equation 2.8.

Under small signal condition, the optical field of the forward-propagating RF-modulated signal by a phase modulator (PM) is given by [108]:

$$
\begin{aligned}
\overline{E_{S}}= & E_{0} \exp \left(j 2 \pi f_{c} t\right)\left[J_{0}(m)+J_{1}(m) \exp \left(j 2 \pi f_{R F} t\right)\right. \\
& \left.-J_{1}(m) \exp \left(-j 2 \pi f_{R F} t\right)\right]
\end{aligned}
$$

where $E_{0}$ represents the optical field at the input of the external modulator, $J_{n}$ denotes the nth-order Bessel function of the first kind with $n=0, \pm 1, m$ is the phase modulation index, and $f_{R F}$ represents the frequency of the input microwave signal.

The Jones vector of the optical field after SBS is:

$$
\overline{E_{S}^{\prime}}=\left[\begin{array}{l}
E_{x} S B S_{\min } \\
E_{y} S B S_{\max }
\end{array}\right]
$$




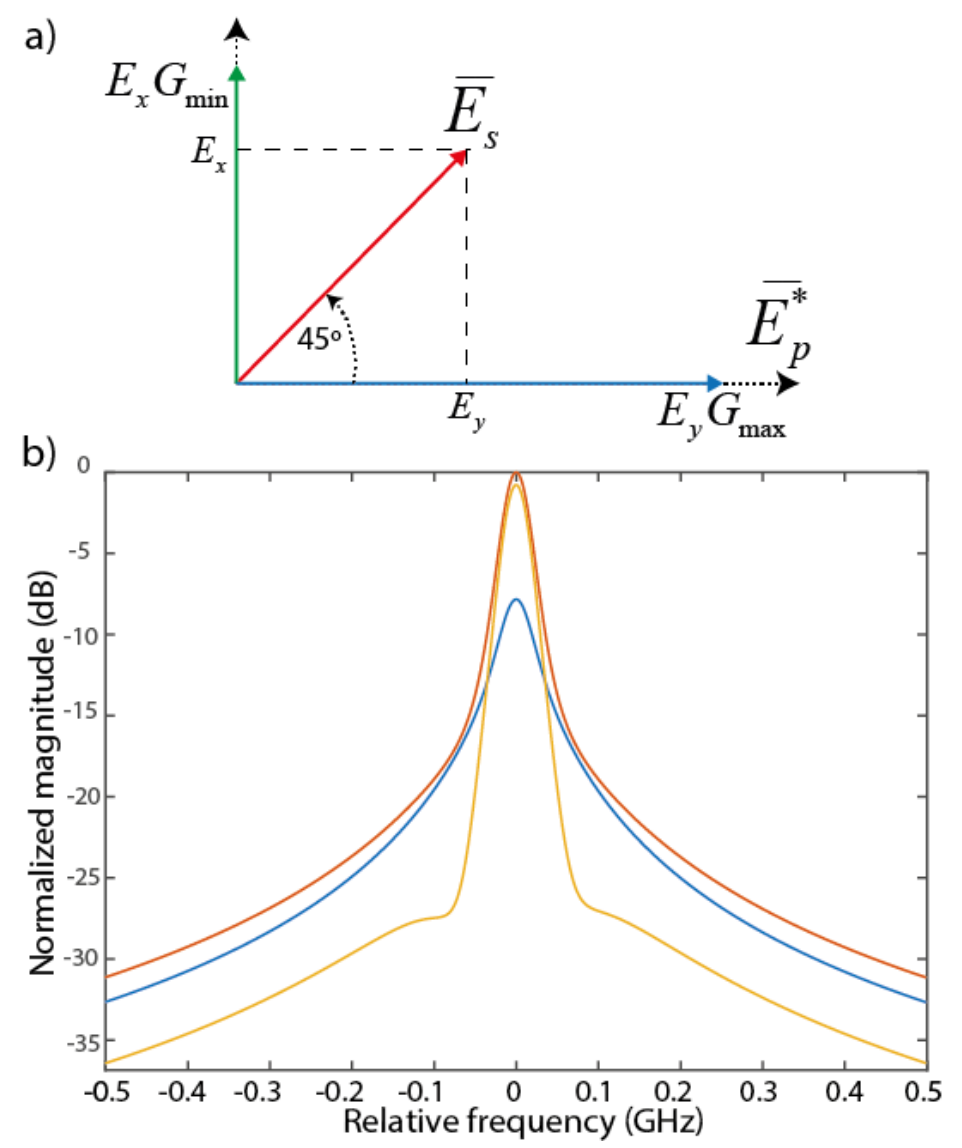

Figure 4.7 (a) Amplified signal on maximum and minimum gain. $\overline{E_{S}}$ is the field of the signal wave before SBS gain. $G_{\max }$ and $G_{\min }$ are the maximum and minimum Brillouin gain in a birefringent fiber. (b) Principle of operation. Frequency response of maximum (red) and minimum (blue) SBS gain and combined response at the output of the detector (yellow).

where $E_{y}$ and $E_{x}$ represents the signal wave projection on the axes of the birefringent fiber, and $S B S_{\max }, S B S_{\min }$ are the maximum and minimum SBS gain/loss, respectively.

$$
\begin{aligned}
E_{x} S B S_{\text {min }}= & \frac{E_{0} \exp \left(j 2 \pi f_{c} t\right)}{\sqrt{2}}\left[J_{0}(m)\right. \\
& +J_{1}(m) \exp \left(j 2 \pi f_{R F} t\right) \exp \left(g_{B}(f) L \zeta\right) \\
& \left.-J_{1}(m) \exp \left(-j 2 \pi f_{R F} t\right) \exp \left(\alpha_{B}(f) L \zeta\right)\right]
\end{aligned}
$$




$$
\begin{aligned}
E_{y} S B S_{\text {max }}= & \frac{E_{0} \exp \left(j 2 \pi f_{c} t\right)}{\sqrt{2}}\left[J_{0}(m)\right. \\
& +J_{1}(m) \exp \left(j 2 \pi f_{R F} t\right) \exp \left(g_{B}(f) L \zeta\right) \\
& \left.-J_{1}(m) \exp \left(-j 2 \pi f_{R F} t\right) \exp \left(\alpha_{B}(f) L \zeta\right)\right]
\end{aligned}
$$

Equation 4.6

where the term $\zeta$ is $2 / 3$ for the maximum gain/loss and $1 / 3$ for the minimum gain/loss in birefringent fiber [17]. The magnitude and phase of SBS gain/loss response due to SBS can be expressed as:

$$
\begin{aligned}
& G_{\max , \min }\left(f_{R F}\right)=\exp \left\{\operatorname{Re}\left[g_{B}\left(f_{R F}\right)\right] L \zeta\right\} \\
& \text { Equation } 4.7 \\
& A_{\text {max,min }}\left(f_{R F}\right)=\exp \left\{\operatorname{Re}\left[\alpha_{B}\left(f_{R F}\right)\right] L \zeta\right\} \\
& \text { Equation } 4.8 \\
& \varphi_{\max , \min }\left(f_{R F}\right)=-\operatorname{Im}\left[g_{B}\left(f_{R F}\right) L \zeta\right]=\operatorname{Im}\left[\alpha_{B}\left(f_{R F}\right) L \zeta\right]
\end{aligned}
$$

To enhance out-of-band rejection, the signal to be filtered is modulated in phase and after the SBS gain/loss process in the birefringent optical fiber, the signal is passed through a polarization beam splitter (PBS), separating the optical field of the signal in two signal waves, $E_{y} S B S_{\max }$ and $E_{x} S B S_{\min }$. Figure 4.8(a) shows the signal experiencing maximum gain/loss and Figure 4.8(b) the signal with minimum gain/loss.

Omitting the DC component as well as higher-order harmonics, the RF power after photodetection of the two optical signals $\underset{\max }{\left(P_{\text {Fout }}, P_{\text {RFout }}\right)}$ is:

$$
\begin{aligned}
P_{\text {RFout }} & \propto\left[\left(E_{y} S B S_{\text {max }}\right)^{*}\left(E_{y} S B S_{\text {max }}\right)^{*}\right]^{2} \\
& \propto\left[\left|G_{\text {max }}\left(f_{R F}\right)\right|^{2}+\left|A_{\text {max }}\left(f_{R F}\right)\right|^{2}-2 \cos \left(2 \varphi_{\text {max }}\right)\right] \\
\underset{P_{\text {min }}}{P_{\text {mout }}} & \propto\left[\left(E_{x} S B S_{\text {min }}\right)^{*}\left(E_{x} S B S_{\text {min }}\right)^{*}\right]^{2} \\
& \propto\left[\left|G_{\text {min }}\left(f_{R F}\right)\right|^{2}+\left|A_{\text {min }}\left(f_{R F}\right)\right|^{2}-2 \cos \left(2 \varphi_{\text {min }}\right)\right]
\end{aligned}
$$

Finally, the filter frequency response based on the SBS gain/loss process in birefringent optical fibers is expressed as,

$$
\begin{aligned}
H\left(f_{R F}\right)= & \eta^{2} H\left(f_{R F}\right)_{\max }-H\left(f_{R F}\right)_{\min } \\
\propto & \eta^{2}\left|G_{\max }\left(f_{R F}\right)\right|^{2}-\left|G_{\min }\left(f_{R F}\right)\right|^{2}+ \\
& \eta^{2}\left|A_{\max }\left(f_{R F}\right)\right|^{2}-\left|A_{\min }\left(f_{R F}\right)\right|^{2}- \\
& 2 \eta^{2} \cos \left(2 \varphi_{\max }\right)-2 \cos \left(2 \varphi_{\min }\right)
\end{aligned}
$$




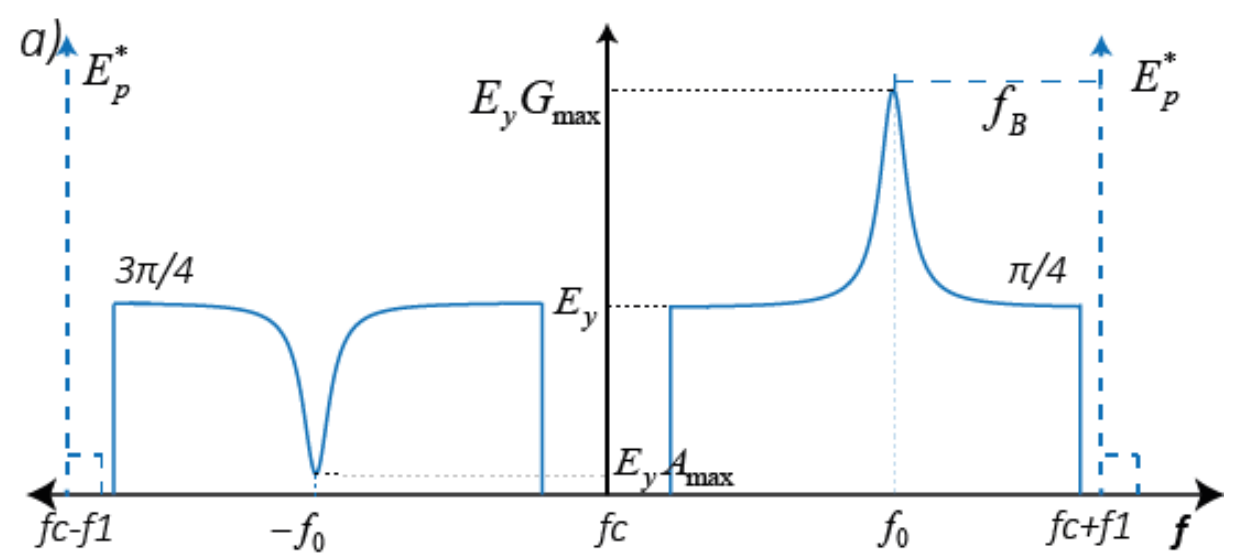

b)

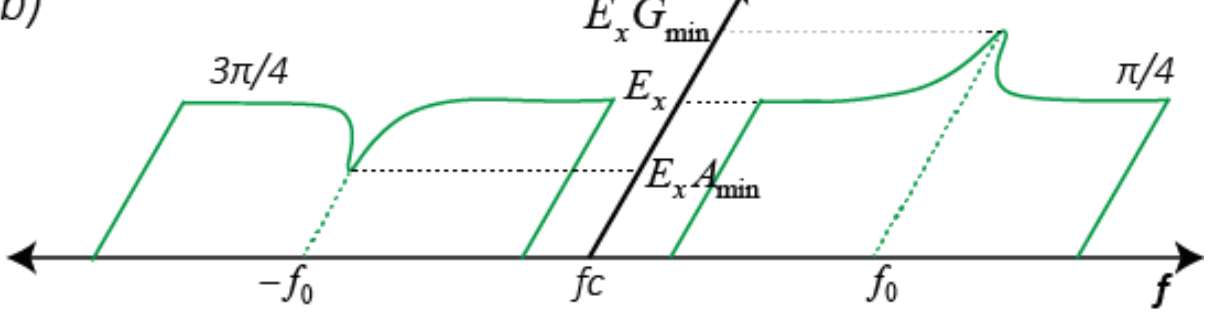

Figure 4.8 Phase-modulated optical signal experiencing SBS. (a) Maximum gain/loss, $E_{y} S B S_{\max }$ and (b) Minimum gain/loss, $E_{x} S B S_{\min } . f_{c} \pm f_{1}$ are the frequencies of the pump waves.

The factor $\eta$ is the optical attenuation that has to be applied to the signal experiencing maximum gain/loss (Figure 4.8(a)) to match the amplitudes of both Brillouin response tails after each photodetector. Figure 4.9 (left) shows the theoretical evolution of the slope of the filter response as a function $\eta$. For a value of $\eta=0 \mathrm{~dB}$, the filter response associated with the maximum SBS gain dominates if the pump power is high enough and the filter slope is the conventional value of around $4 \mathrm{~dB} /$ oct from the natural Lorentzian response. Beyond a value of $\eta=3.1 \mathrm{~dB}$, lobes appear in the filter response that degrade out-of-band rejection. Figure 4.9 (right) shows the additional microwave loss in the filter response induced by the attenuation $\eta$. The additional microwave loss follows a linear trend, falling as expected $2 \mathrm{~dB}$ for each optical $\mathrm{dB}$ until a factor around $\eta=1.5$ $\mathrm{dB}$, where the effect of the subtraction between the two filter responses starts to dominate. 


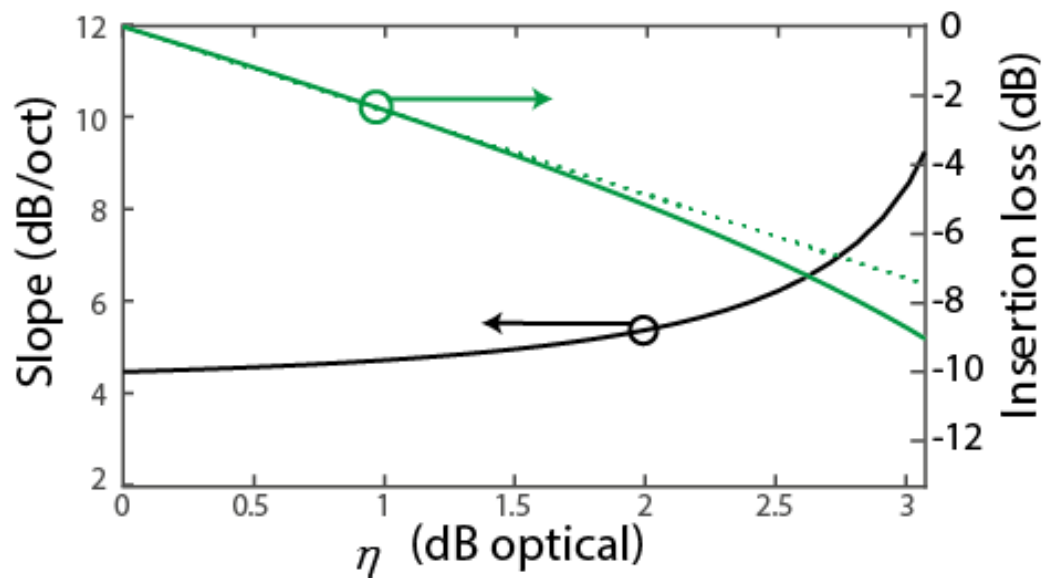

Figure 4.9 Slope of the Brillouin-based photonic microwave filter (left) and microwave additional loss (right) versus factor $\eta$ for a pump signal of $12.5 \mathrm{dBm}$ that induced a maximum SBS gain value of $\left|G_{\max }\left(f_{R F}\right)\right|^{2}=14 \mathrm{~dB}$.

\subsubsection{Experimental Results}

Figure 4.10 shows the experimental setup. The light from a DFB source at $1548 \mathrm{~nm}(12.5$ $\mathrm{dBm}$ ) is divided by a directional coupler 50/50. The upper path of the setup was used to generate the pump while the lower one corresponds to the signal.

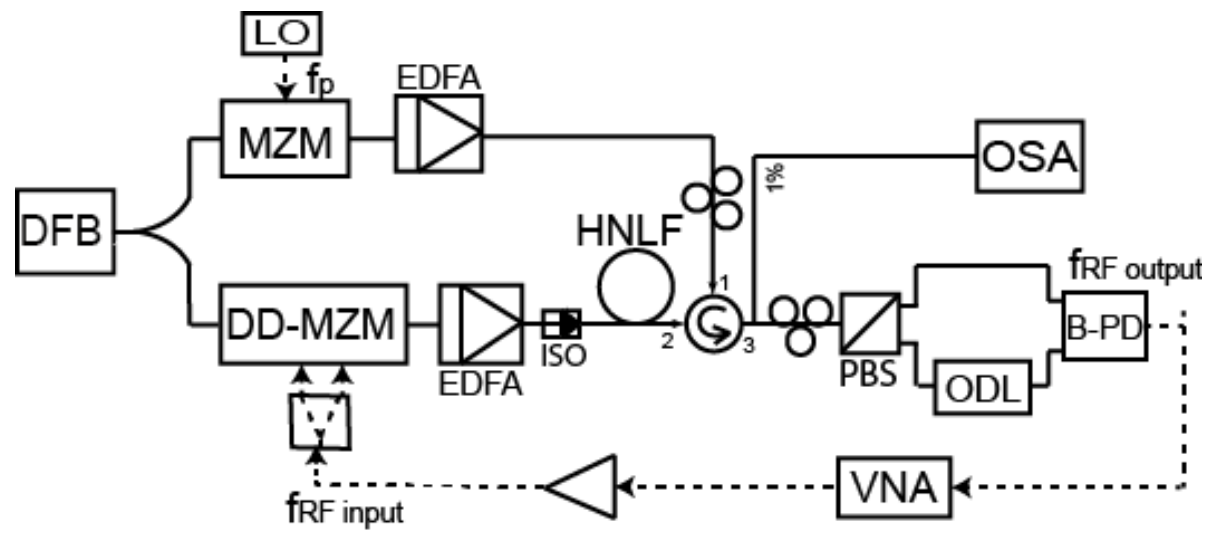

Figure 4.10 Block diagram of the experimental setup. DFB: distributed feedback laser, MZM:

Mach-Zehnder modulator, DD-MZM: Dual-Drive MZM, ISO: isolator, PBS: polarization bean splitter, ODL: optical delay line, B-PB: balanced photo detector, VNA: vector network analyzer,

LO: local oscillator. 
In the upper path, the optical signal is introduced in an MZM biased at the minimum transmission point (MITB), generating a DSB-SC modulation. The pump signal at $f_{c}+$ $f_{1}$ induces an SBS gain response over the upper sideband and the pump at $f_{c}-f_{1}$ induces an SBS loss response to the lower sideband. Optical amplification is used to boost the pump signal and, finally, an optical circulator directs the pump toward the nonlinear medium (a reel of $1 \mathrm{~km}$-long HNLF with Brillouin linewidth $\Delta v_{B}=40 \mathrm{MHz}$ and Brillouin frequency shift $v_{B}=9.64 \mathrm{GHz}$ ).

In the lower path, the data signal is phase modulated using a DD-MZM and a microwave hybrid coupler. The DD-MZM is biased at the maximum transmission point (MATB). An isolator (ISO) is used to eliminate the pump signal after it crossed through the HNLF. The modulated signal is boosted by optical amplification and it crosses the HNLF. Through an optical circulator, it reaches a polarization beam splitter (PBS). The SOP of the signal wave was adjusted to $45^{\circ}$ in relation to the maximum and minimum SBS gain axes by means of a polarization controller as was shown in Figure 4.7(a). Finally, both signals are detected by a balanced photodiode. The filter frequency response can be tuned by changing the frequency of the single local oscillator.

Figure 4.11 shows the filter response measured for an SBS-based filter with phase modulation experiencing maximum (red) and minimum (green) SBS gain/loss and with the system described in Figure 4.10. There is a good agreement between theory and experimental results.

It can be seen in Figure 4.11 that the slope of the Lorentzian Brillouin response is enhanced. The filter response shows a mean filter slope of $8.3 \mathrm{~dB} /$ oct and an out-of-band rejection ratio of around $40 \mathrm{~dB}$. For the sake of comparison, a conventional SBS-based filter response of the same gain shows a slope of $4.8 \mathrm{~dB} /$ oct. 

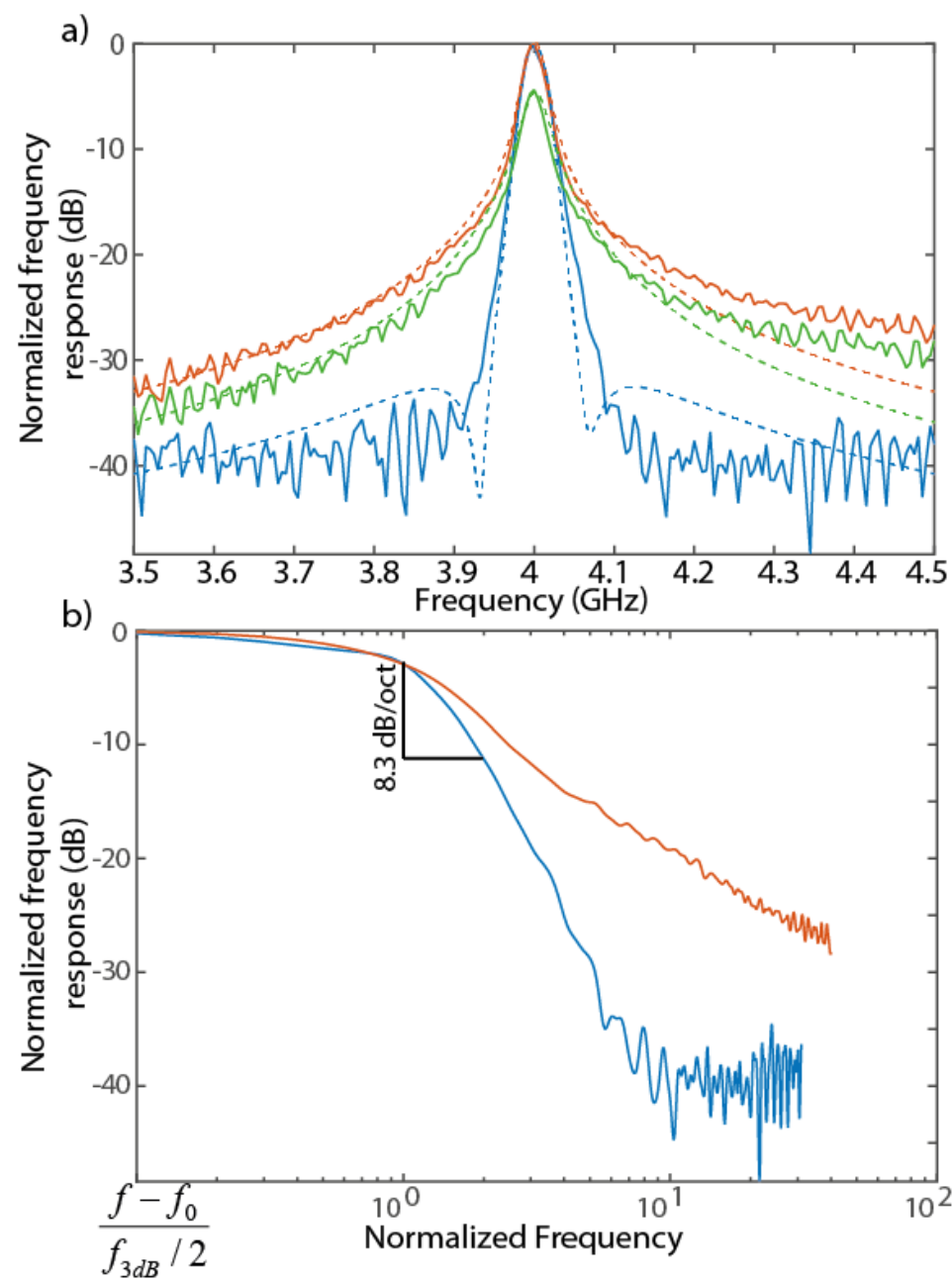

Figure 4.11 (a) Experimental SBS-based filter response: with phase modulation and pump power of $12 \mathrm{dBm}$ experiencing maximum (red) and minimum (green) SBS gain/loss; SBS-based photonic microwave filter exploiting fiber birefringence and pump power of $14.5 \mathrm{dBm}$ (blue). Theoretical SBS-based filter response: with phase modulation experiencing maximum (red dotted) and minimum (green dotted) SBS gain/loss; SBS gain/loss process in presence of birefringent optical fiber in combination with balanced detection (blue dotted). (b) Bode diagram of the experimental SBSbased filter response.

Figure 4.12 compares in terms of bandwidth and shape factor a conventional SBS-based filter with phase modulation and the new technique proposed here for different pump powers. Figure 4.12(a) shows that the bandwidth at $-20 \mathrm{~dB}$ is considerably reduced with 
the new technique whilst the bandwidth at $-3 \mathrm{~dB}$ remains quite constant. Figure 4.12(b) shows that the filter shape factor is enhanced with the proposed method.
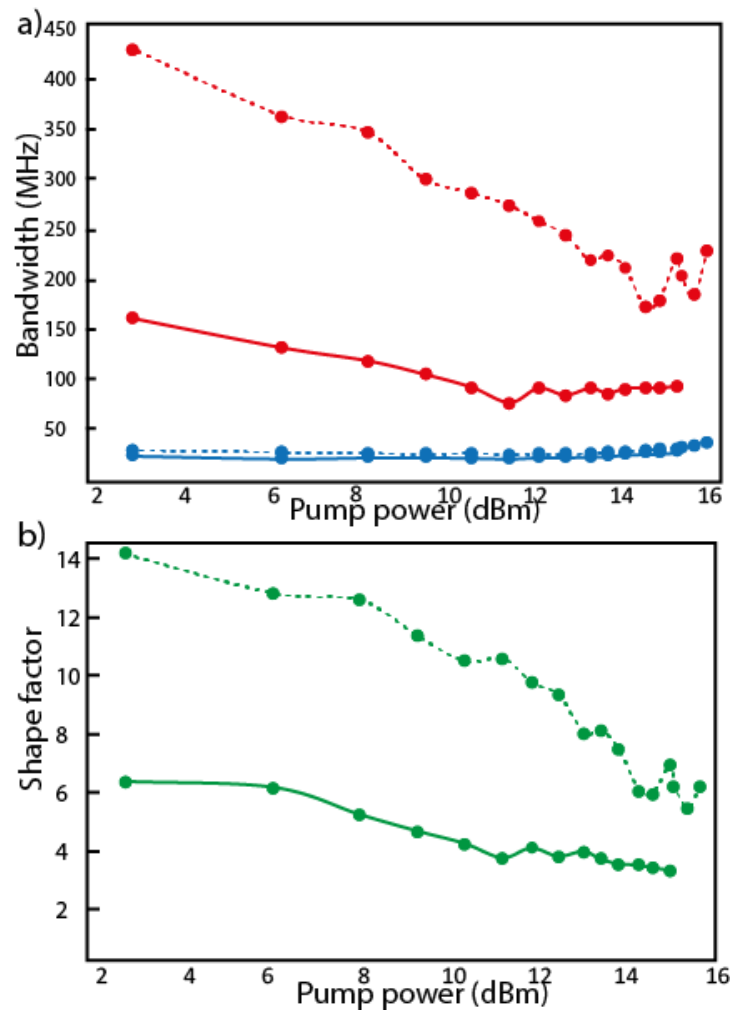

Figure 4.12 Comparison between a conventional phase-modulated SBS microwave filter (dotted) and the new technique (solid). (a) Bandwidth of the filter response. At -20 dB (red) and -3 dB (blue). (b) Shape factor.

\subsubsection{Conclusion}

A method to enhance the slope of SBS-based photonic microwave filters has been proposed. By exploiting the usually deleterious effect induced by birefringent fiber on Brillouin processing it has been shown that maximum and minimum Brillouin gain/loss can be used to enhance the filter response. A filter with a slope of $8.3 \mathrm{~dB} /$ oct and an out-ofband rejection of $40 \mathrm{~dB}$ has been demonstrated. The filter selectivity is enhanced with a simple scheme and just a single stage and one pump wave. 


\subsection{Wide tunable photonic microwave notch filter based on Brillouin po- larization controller.}

\subsubsection{Introduction}

In this section a new photonic microwave notch filter is presented. The principle operation is based on the new all-optical polarization control presented in Chapter 3. Here, it is shown that it can be used to implement notch filters with high suppression.

\subsubsection{Principle of operation}

The technique is based on exploiting the polarization control capability demonstrated on Chapter 3, to cross rotate the SOP of one of the sidebands to eliminate the signal with a polarizer. If the RF signal is modulated in SSB, it results in the implementation of a tunable notch filter. It is shown in Figure 4.13 where the sideband, for the sake of simplicity, has been represented by three Dirac deltas. The pump is set to perform a circular retarder. The pump SOP must be circular. The signal polarization rotates toward linear horizontal SOP (Figure 3.2(b) (blue)) until it matches with orthogonal polarization of the polarizer.
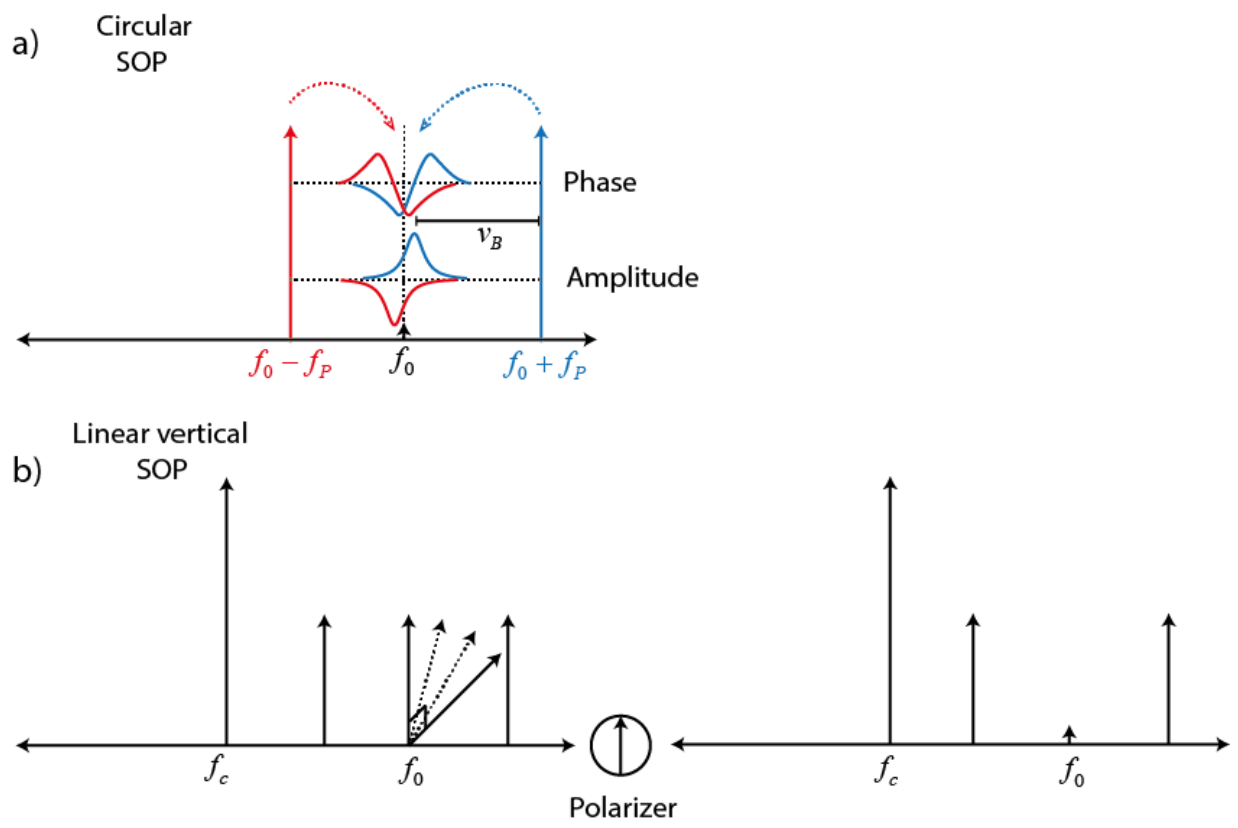

Figure 4.13. Operational principle of the SBS-based polarization controller filter. a) Pump frequency response. b) Signal frequency response. 


\subsubsection{Experimental results}

The experimental setup used to validate the concept of the SBS-based all-optical notch filter is shown in Figure 4.14. An optical signal $(1548 \mathrm{~nm})$ is split into two paths. The

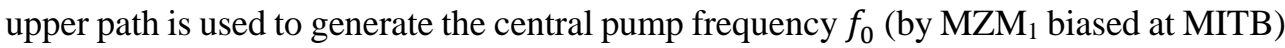
of the Brillouin polarization control stage that acts as a circular retarder. It is implemented by two Mach-Zehnder modulators ( $\mathrm{MZM}_{1}$ and $\mathrm{MZM}_{2}$ ) biased at MITB and fed by two microwave oscillators with frequencies $f_{p 1}(9.607 \mathrm{GHz})$ and $f_{p 2}(9.677 \mathrm{GHz})$. A circular $\left(\mathrm{OC}_{1}\right)$ directs the SBS pump toward the circular retarder through 1-km HLNF. In the lower path, the intensity optical is modulated by RF signal through DD-MZM at quadrature bias, single sideband with carrier $\mathrm{SSB}+\mathrm{C}$. The carrier power at input fiber is of $-8 \mathrm{~dB}$. The SBS process occurred in the HNLF between the RF-modulated signal and the pump wave. The Brillouin frequency shift of the HLNF was 9.64 GHz. The Brillouin polarization control was optimized using a polarization controller by adjusting the polarization of the two counterpropagating signals at frequencies $f_{p 1}$ and $f_{p 2}$ to circular SOP. An FBG in reflection mode (bandwidth of $12.5 \mathrm{GHz}$ ) is used to filter out backward residual pump waves. The pump power is adjusted by VOAs to rotate the polarization of the signal until it is converted to the orthogonal SOP in relation to the polarizer transmission axis. The power of the pump set at $8 \mathrm{dBm}$, reaching a retardance measured using the Poincaré sphere method [83] of $\pi$. The filtered modulated signal reached a PIN photodiode. Finally, the frequency response of the filter is measured with a VNA.

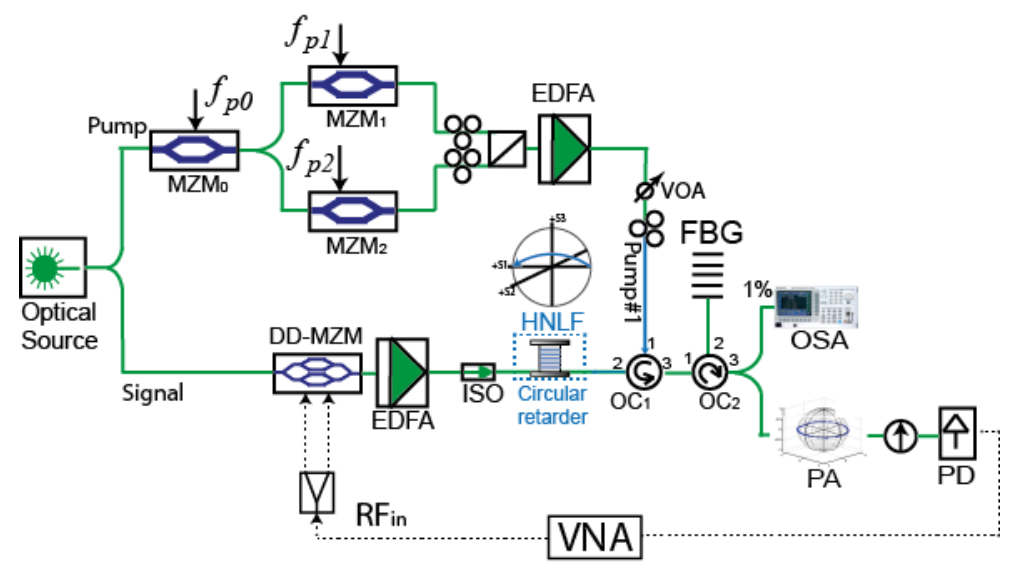

Figure 4.14 Block diagram of the experimental setup of the all-optical polarization controller filter. HLNF: Highly nonlinear fiber. OC: Optical circulator; ISO: Isolator. VOA: Variable Optical Attenuator. FBG: Fiber Bragg grating. OSA: Optical spectrum analyzer. PA: Polarization analyzer. PD: photodetector. VNA: vector network analyzer. 
Figure 4.15 shows the normalized response of a notch filter designed to operate with a center frequency of $5 \mathrm{GHz}$, when the modulator signal DD-MZM acted as an upper side band carrier with LSB+C $\left(V_{\text {bias }}=\pi / 2\right)$.

More interestingly, the notch exhibits a depth of $67 \mathrm{~dB}$, which is, to the best of our knowledge, the best suppression reported with an SBS notch filter, which is slightly higher than that record reached by [101].

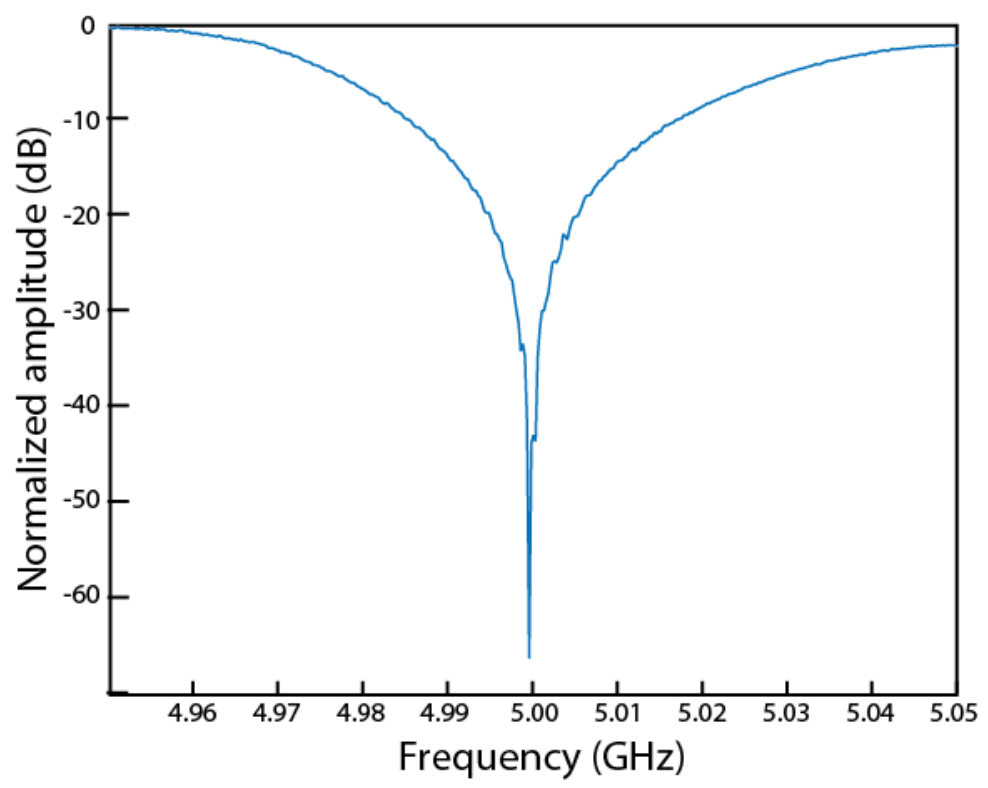

Figure 4.15 Measured normalized response of the photonic microwave notch filter.

The ability to tune the notch filter over a wideband frequency range was experimentally demonstrated. This was realized by changing the pump frequency $f_{p 0}$ driving MZMo. The Figure 4.16 shows the measured frequency response results for the notch filter. The tuning of the notch filter frequency from $2-6 \mathrm{GHz}$ is demonstrated. This frequency range was chosen due to the unavailability of a microwave oscillator with a higher range in our laboratory. 


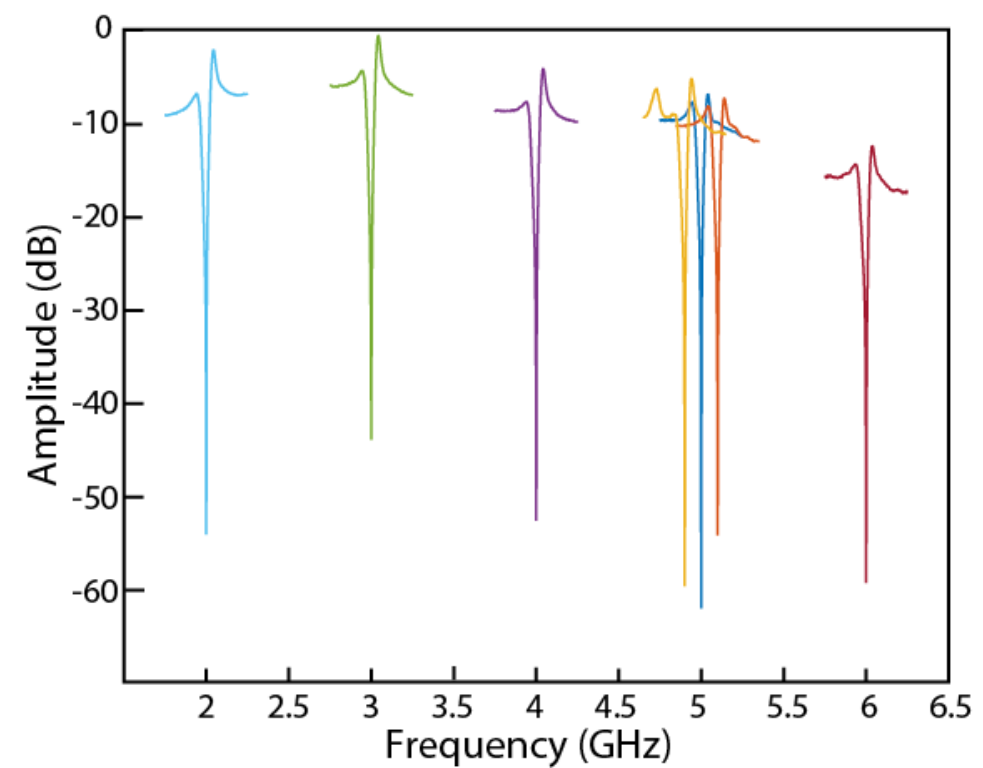

Figure 4.16 Measured frequency response of the tunable notch filter response by changing the frequency pump $f_{p 0}$.

\subsubsection{Conclusion}

A new structure that implements a notch filter photonic microwave filter that exhibits a very deep notch $(67 \mathrm{~dB})$ and has the capability to provide continuous tuning has been presented. Unlike other notch filters, the large depth of the filter is performed in the optical domain. It is based on the new Brillouin polarization control technique presented in Chapter 3. 


\section{Chapter 5}

\section{Conclusions and future outlook}

In this PhD Thesis, the application of SBS has been studied. In particular, new schemes based on SBS have been developed for all-optical polarization control and photonics microwave filtering.

In the area of polarization control, a new technique has been presented. Unlike previous works based on polarization attraction, a different approach has been proposed that relies on the phase shifting capability of SBS. This allows the implementation of nonlinear wavelength-selective analogues of waveplates. The retardance induced by SBS, measured using the Poincare sphere method, has shown a linear variation with pump power, showing a trending similar to the behavior of the SBS gain. A full rotation $(2 \pi)$ on the equator of the Poincare sphere has been achieved with a pump power around of $11 \mathrm{dBm}$. The retardance variations as a function of pump power is similar for input signal powers between $0.08 \mathrm{~mW}$ to $12.6 \mathrm{~mW}$, showing that the effect has little dependence on the power of the input signal. When the signal SOP is rotated to its farther point $(\Gamma=\pi)$ over Poincaré sphere, the DOP remains above $98 \%$. In fact, a full rotation, i.e. beyond to $\pi$, is not needed because the rotation sense is inverted by swapping the frequencies of the local oscillator. Results show a variation of the insertion loss of 1.2 dB. A Multi-tone pump configuration allows control the retardance bandwidth. Using an electrical method, the NF maximum variation has been measured showing a degradation of just $1.5 \mathrm{~dB}$ for a full rotation $(\Gamma=2 \pi)$. 
With the aim of fulfilling real masks for microwave filter responses, the performance of SBS-based photonic microwave filters need to be enhanced. Thus, efforts have been devoted to push the performance of SBS-based microwave filters closer to real industry needs. This requires engineering the slope of the filter, i.e. enhancement of the filter order.

First, a technique used to attenuate the slowly decaying tail of the Lorentzian response based on combining a SBS gain response with two frequencies-offset SBS loss responses has been proposed. An experimental setup has been used to validate this concept. The data signal was phase modulated and when the SBS introduce a gain/loss over the sidebands, which breaks out-of-phase conditions between sidebands, by enhancing the filter slope while keeping the rejection ratio. The results show an enhanced selectivity of the filter response and no requiring high SBS gain. The experimental filter response shows an improvement of $16.7 \mathrm{~dB} /$ oct. It is a significant improvement over the $3.79 \mathrm{~dB} /$ oct and $4.08 \mathrm{~dB} /$ oct of the theoretical response with a single pump of 5 and $15 \mathrm{~dB}$ gain, respectively. Finally, a flat-top filter response has been developed by a feedback algorithm and sweeping the pump frequencies.

Second, a new approach for improving skirt selectivity, which simplify the architecture and it is easy tuning by mean of a single local oscillator. This technique use the maximum and minimum Brillouin induced when pump wave is launched into birefringent fiber. Experimental results show a filter with an out-of-band rejection of $40 \mathrm{~dB}$ and an improved slope of $8.3 \mathrm{~dB} /$ oct.

Third, a new technique based on the new polarization controller based on SBS, has been used to improve the suppression of SBS notch filters. This technique uses an optical polarizer to reach a very deep $(67 \mathrm{~dB})$ suppression of unwanted signals. The notch frequency $f_{0}$ can be continuously tuned by just one microwave oscillator over a wide frequency band.

Further work is needed to keep advancing the research lines followed in the Thesis. In relation to the all-optical polarization controller, further work is needed to find new applications for it. In addition, its applicability in integrated structures to allow new optical processing functionalities in PICs. For example, in integrated structures based on chalcogenide glass. In relation to photonic microwave filtering, the SBS-based experimental schemes presented above have integration potential. Further work is needed to enhance the performance of integrated photonic microwave filters and make them closer to market needs. SBS is a great candidate to lead this approach. 


\section{Appendix I Polarization Optics}

\section{I.1 Polarization Optics}

A brief summary of the basic concepts related to polarization optics to make the Thesis document self-contained and ease reading is reported in this Annex.

Considering a wave propagating in the direction $O z$ (Figure I.1), the standard expression for the electric field of an electromagnetic wave of angular frequency $\omega$ propagating in an optical medium is:

$$
\boldsymbol{E}(\mathbf{z}, \boldsymbol{t})=\boldsymbol{E} \exp (i \omega t)
$$

Equation I.1

where $\boldsymbol{E}(\boldsymbol{z}, \boldsymbol{t})$ and $\boldsymbol{E}$ are complex vectors. $\boldsymbol{E}$ is a vector in the $x y$ plane. The $\boldsymbol{E}$ field may be considered as the sum of its two linearly polarized components in $O x$ and $O y$ directions $\left(E_{x}, E_{y}\right)$. The complete solution to the wave equation by means of two waves is given by

$$
\begin{array}{ll}
\boldsymbol{E}(\boldsymbol{z}, \boldsymbol{t})=E_{x}(z, t) \hat{e}_{x}+E_{y}(z, t) \hat{e}_{y} & \text { Equation I.2 } \\
E_{x}=A_{x} \cos \left(\omega t-k z+\varphi_{x}\right) & \text { Equation I.3 } \\
E_{y}=A_{y} \cos \left(\omega t-k z+\varphi_{y}\right) & \text { Equation I.4 }
\end{array}
$$

where $A_{x}$ and $A_{y}$ are field amplitude in each axis, $\varphi_{x}$ and $\varphi_{y}$ are arbitrary phase angles. Each vector oscillates sinusoidally with the time alone of the fiber (Figure I.1). 


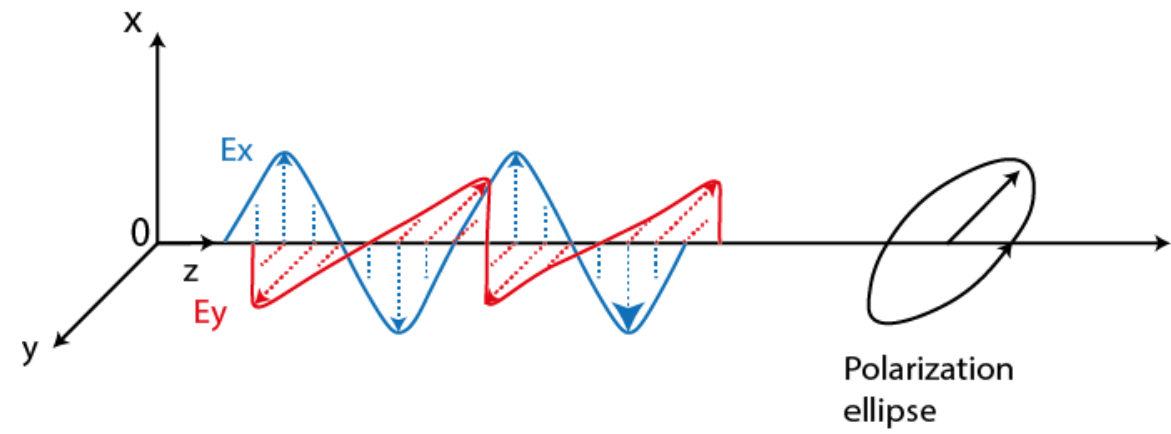

Figure I.1 Electric field components for polarized wave

Choosing any point along of the fiber and changing the starting time $(t)$ such as $\varphi=$ $\varphi_{y}-\varphi_{x}$, Equation I.3 and Equation I.4 can be re-written as

$$
\begin{aligned}
& E_{x}=A_{x} \cos (\omega t) \\
& E_{y}=A_{y} \cos (\omega t+\varphi)
\end{aligned}
$$

Equation I.5

Equation I.6

The tip of the resultant vector of the electric field due to the sum of $E_{x}$ and $E_{y}$ at a fixed point along of the fiber rotates periodically in the $x z$ plane tracing an ellipse, accomplishing a full ellipse in a total time equal to the period of the wave. The ellipse Cartesian equation is given by

$$
\frac{E_{x}^{2}}{A_{x}^{2}}+\frac{E_{y}^{2}}{A_{y}^{2}}-\frac{2 \cos \varphi}{A_{x} A_{y}} E_{x} E_{y}=\sin ^{2} \varphi
$$

Equation I.7

Equation I.7 describes an elliptically polarized wave composed by its two electric field components $\left(E_{x}, E_{y}\right)$, if the phase difference $(\varphi)$ is constant in the time. Assuming that the mutual coherence between the two waves $\left(E_{x}, E_{y}\right)$ is good, we can say that elliptical polarization is the more general state of the polarization of the light [64]. The state of the polarization (SOP) of the light is linear when this ellipse becomes straight line; that is,

Linearly polarized in $O y$ direction:

$A_{x} \neq 0 ; A_{y}=0$

Linearly polarized in $O x$ direction:

$A_{x}=0 ; A_{y} \neq 0$

or 
$\varphi=m \pi$

where $m$ is any integer, including zero. Meanwhile, the ellipse converts a circle if

$A_{x}=A_{y}$

and

$\varphi=\frac{(2 m+1) \pi}{2}$

where $m$ determines the sense of rotation. A wave is right-hand or left-hand circularly polarized when $m$ is even and odd, respectively. The light is circularly polarized when the component waves have equal amplitudes and are in phase quadrature.

A birefringent medium is an anisotropic medium with different refractive index in its optical axes, i.e., a medium in which orthogonal components of the wave travel at different velocities. Thus, the SOP of the light will be dependent of the propagation distance.

\section{I.1.1 Jones Vector}

In an optical medium, the Jones calculus ${ }^{4}$ is used to describe polarized light. The Jones vector describes both amplitude and phase of the components of the electric field for monochromatic waves as a two dimensional complex vector:

$$
\boldsymbol{E}=\left(\begin{array}{l}
\boldsymbol{E}_{\boldsymbol{x}} \\
\boldsymbol{E}_{\boldsymbol{y}}
\end{array}\right) \exp (i \omega t)=\left(\begin{array}{l}
A_{x} \exp \left(i \varphi_{x}\right) \\
A_{y} \exp \left(i \varphi_{y}\right)
\end{array}\right) \exp (i \omega t)
$$

Equation I.8

The effect that a medium induces on a signal is represented in Jones calculus by a matrix, the Jones matrix, i.e. they described the polarization conversion as a consequence of traversing the medium, as shown in Equation I.9

$$
\left(\begin{array}{l}
E_{x}^{\text {out }} \\
E_{y}^{\text {out }}
\end{array}\right)=(M)\left(\begin{array}{c}
E_{x}^{\text {in }} \\
E_{y}^{\text {in }}
\end{array}\right)
$$

${ }^{4}$ R. C. Jones proposed this method in 1941 [111]. 


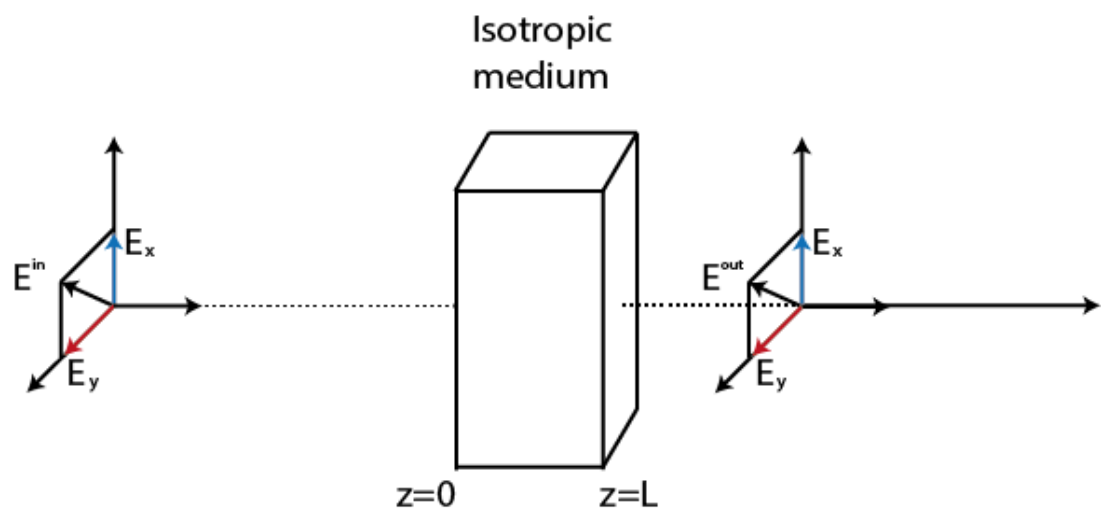

Figure I.2 SOP of the light at the output of an isotropic medium.

where $(M)$ represents the optical. In general, $M$ is a $2 \times 2$ matrix whose elements are complex numbers. The Jones matrix is also equivalent to a rotation of the Poincaré sphere [109], which will be present below.

A typical device in polarization control is the polarizer. It is an optical device, which induces a strong attenuation to one polarization component. Therefore, it allows passing only one component with a defined SOP. The relation between output and input intensity is called transmittance. The maximum transmittance matched with the transmission axis, whereas the minimum one corresponds to the absorption axis. The transmission and absorption axes are orthogonal.

The orientation of the lineal polarizer is determined by azimuth angle $(\theta)$ formed from its transmission axis. The matrix $(M)$ that represents the different forms of a polarizer is show in Table I.1.

Some of the main mechanisms that modifies the SOP of the light are absorption, reflection, refraction, birefringence and scattering.

Another relevant component that is a key concept in some sections of the Thesis is the waveplate. Waveplates convert polarization, i.e. it changes the SOP of the signal [64].

If a wave is linearly polarized at $45^{\circ}$ to the optic axis, and the output has circular SOP, the device is called a quarterwave plate retarder and the induced phase difference (retardance) between components is of $\varphi=\pi / 2$ (Figure I.3 (b)). Meanwhile, if the phase difference inserted is $\varphi=\pi$ (Figure I.3 (a)), the light at the output is linearly polarized but with $-45^{\circ}$. In this case, the retarder is a halfwave plate. Figure I.1Table I.1 show the Jones matrix for the quarterwave and halfwave waveplates. 

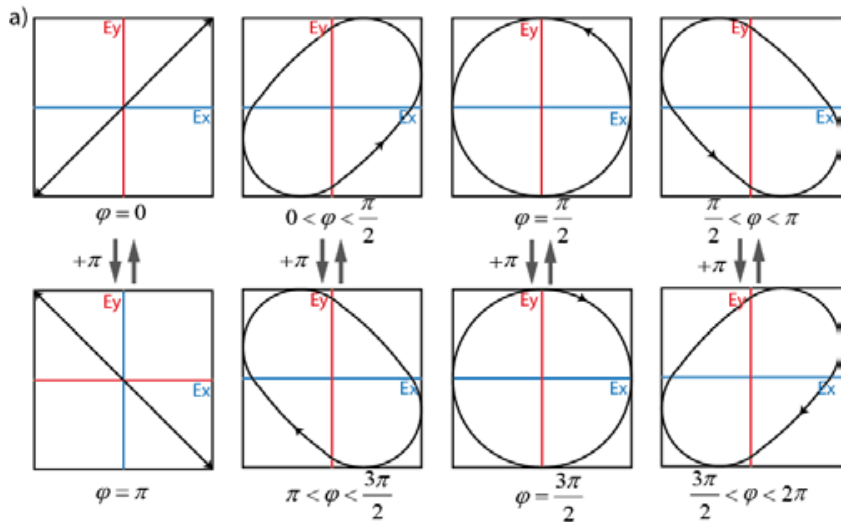

b)
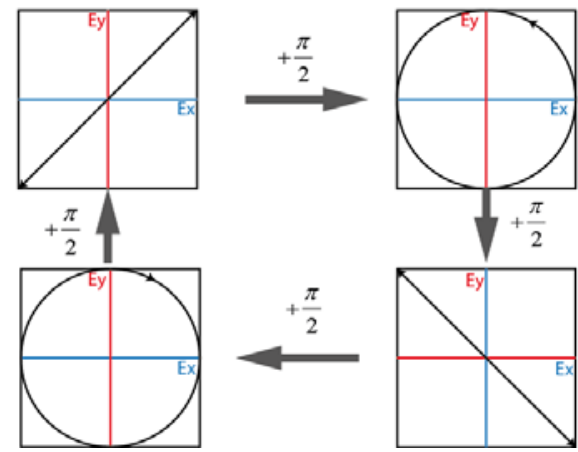

Figure I.3 Retarder (a) halfwave and (b) quarterwave

Table I.1 Jones matrix of a linear optical medium

\begin{tabular}{|l|c|}
\hline \multicolumn{1}{|c|}{ Linear optical medium } & Jones matrix \\
\hline Horizontal linear polarizer & $\left(\begin{array}{ll}1 & 0 \\
0 & 0\end{array}\right)$ \\
\hline Vertical linear polarizer & $\left(\begin{array}{ll}0 & 0 \\
0 & 1\end{array}\right)$ \\
\hline Linear polarizer at $45^{\circ}$ & $\frac{1}{2}\left(\begin{array}{ll}1 & 1 \\
1 & 1\end{array}\right)$ \\
\hline Linear polarizer at $-45^{\circ}$ & $\frac{1}{2}\left(\begin{array}{rr}1 & -1 \\
-1 & 1\end{array}\right)$ \\
\hline Quarterwave retarder & $\left(\begin{array}{ll}1 & 0 \\
0 & \exp \left(-i \frac{\pi}{2}\right)\end{array}\right)$ \\
\hline Halfwave retarder & $\left(\begin{array}{ll}1 & \exp (-i \pi)\end{array}\right)$ \\
\hline
\end{tabular}


Other polarization states can be handled through rotation matrices,

$$
R(\theta)=\left(\begin{array}{rr}
\cos \theta & \sin \theta \\
-\sin \theta & \cos \theta
\end{array}\right)
$$

Equation I.10

where $\theta$, e.g. is the polarizer angle in relation to the x-axis of the base system. The transformation matrix of a linear polarizer to an $\theta$ angle is given by

$$
J_{\theta}=R(-\theta) J^{\prime} R(\theta)
$$

where $J^{\prime}$ is the Jones matrix of horizontal linear polarizer (Table I.1). For the case of a quarterwave retarder $(\varphi=\pi / 2)$ whose fast axis is matched with the $\mathrm{x}$-axis, the retarder matrix is given by

$$
\begin{aligned}
J_{\theta} & =R(-\theta)\left(\begin{array}{cc}
1 & \exp \left(-i \frac{\pi}{2}\right) \\
0 & \exp
\end{array}\right) R(\theta) \\
& =\left(\begin{array}{cc}
\exp (i \varphi) \cos ^{2} \theta+\sin ^{2} \theta & (\exp (i \varphi)-1) \cos \theta \sin \theta \\
(\exp (i \varphi)-1) \cos \theta \sin \theta & \exp (i \varphi) \sin ^{2} \theta+\cos ^{2} \theta
\end{array}\right)
\end{aligned}
$$

Equation I.12

\section{I.1.2. Stokes parameters}

Another representation of the SOP is based on the Stokes parameters. This calculus is based on a four-element vector.

$$
S=\left(\begin{array}{c}
S_{0} \\
S_{1} \\
S_{2} \\
S_{3}
\end{array}\right)=\left(\begin{array}{c}
A_{x}^{2}+A_{y}^{2} \\
A_{x}^{2}-A_{y}^{2} \\
2 A_{x} A_{y} \cos \varphi \\
2 A_{x} A_{y} \sin \varphi
\end{array}\right)
$$

The Stokes representation of common states of polarization are showed in Table I.2. The parameters are not independent of each other. For polarized light only three parameters are independent.

$$
S_{0}^{2}=S_{1}^{2}+S_{2}^{2}+S_{3}^{2}
$$

where $S_{0}^{2}$ is the total light intensity.

A related concept is the degree of polarization (DOP). For a fully polarized light DOP is $100 \%$, whereas for partially polarized light, since Equation I.14, the DOP is given by

$$
D O P=\frac{S_{1}^{2}+S_{2}^{2}+S_{3}^{2}}{S_{0}^{2}} 100
$$


Table I.2 State of polarization of the light based in Jones and Stokes vector

\begin{tabular}{|l|c|c|}
\hline \multicolumn{1}{|c|}{ State of polarization } & Jones vector & Stokes vector \\
\hline Horizontally polarized & $\left(\begin{array}{l}1 \\
0\end{array}\right)$ & $\left(\begin{array}{l}1 \\
1 \\
0 \\
0\end{array}\right)$ \\
\hline Vertically polarized & $\left(\begin{array}{l}0 \\
1\end{array}\right)$ & $\left(\begin{array}{c}1 \\
-1 \\
0 \\
0\end{array}\right)$ \\
\hline Linearly polarized at $45^{\circ}$ & $\frac{1}{\sqrt{2}}\left(\begin{array}{l}1 \\
1\end{array}\right)$ & $\left(\begin{array}{c}1 \\
0 \\
1 \\
0\end{array}\right)$ \\
\hline Linearly polarized at $45^{\circ}$ & $\frac{1}{\sqrt{2}}\left(\begin{array}{c}1 \\
-1\end{array}\right)$ & $\left(\begin{array}{c}1 \\
0 \\
-1 \\
0\end{array}\right)$ \\
\hline Right-handed circularly & $\frac{1}{\sqrt{2}}\left(\begin{array}{l}1 \\
i\end{array}\right)$ & $\left(\begin{array}{c}1 \\
0 \\
0 \\
1\end{array}\right)$ \\
polarized & $\frac{1}{\sqrt{2}}\left(\begin{array}{c}1 \\
-i\end{array}\right)$ & $\left(\begin{array}{c}1 \\
0 \\
0 \\
-1\end{array}\right)$ \\
\hline Left-handed circularly \\
polarized
\end{tabular}

\section{I.1.3. Poincaré sphere}

A geometrical representation of the Stokes space can help to understand polarization effects. It is known as the Poincaré sphere [109], where $S_{1}, S_{2}$ and $S_{3}$ are the Cartesian coordinates of a point on the sphere (Figure I.4). The SOP of the light change from one SOP to another when crosses through an optical medium, which corresponds to a rotation on the sphere. The relation between angles (rotations) on the Poincaré sphere and Stokes parameters is given by, 


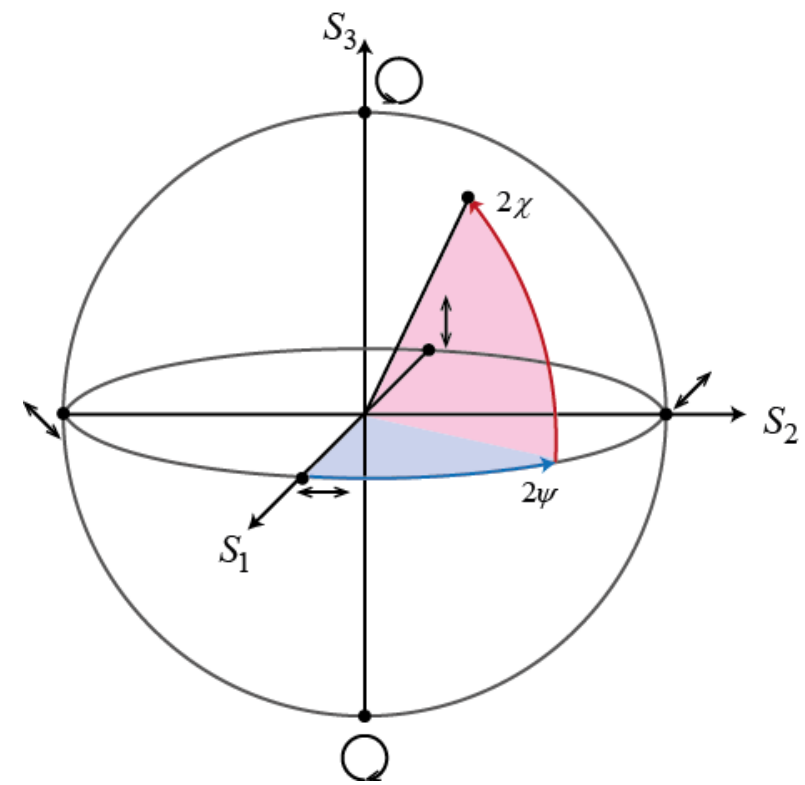

Figure I.4. Poincare sphere. Cartesians coordinates formed by three Stokes parameters $\left(S_{1}, S_{2}, S_{3}\right)$

$S_{0}=I$

$S_{1}=I_{0} \cos 2 \psi \cos 2 \chi$

$S_{2}=I_{0} \sin 2 \psi \cos 2 \chi$

Equation I.16

$S_{3}=I_{0} \sin 2 \chi$

where $I_{0}, 2 \psi$ and $2 \chi$ are the spherical coordinates of the SOP in the three-dimensional space of the Stokes parameters $\left(S_{1}, S_{2}, S_{3}\right)$. The parameters $\psi$ and $\chi$ represent the inclination of the major axis of the polarization ellipse and ellipticity, respectively.

The most relevant SOPs (points on the sphere) are shown in the below table. 
Table I.3 SOP of the light

\begin{tabular}{|c|c|c|}
\hline SOP of the light & $\begin{array}{c}\text { Inclination of the polari- } \\
\text { zation ellipse }\end{array}$ & Ellipticity \\
\hline Lineal polarized at $0^{\circ}$ & $\psi=0$ & $\chi=0$ \\
\hline Lineal polarized at $90^{\circ}$ & $\psi=\frac{\pi}{2}$ & $\chi=0$ \\
\hline $\begin{array}{c}\text { Linearly polarized } \\
\text { polarized }\end{array}$ & $0 \leq 2 \psi \leq 2 \pi$ & $\chi=\frac{\pi}{4}$ \\
\hline $\begin{array}{c}\text { Right-handed circularly } \\
\text { polarized }\end{array}$ & - & $\chi=-\frac{\pi}{4}$ \\
\hline
\end{tabular}




\section{List of publications}

\section{Journal publications}

- D. Samaniego, B. Vidal, "Photonic microwave filter with steep skirt selectivity based on stimulated Brillouin scattering”, IEEE Photonics Journal, vol. 8, no. 6, pp. 5502307, December 2016.

- D. Samaniego, B. Vidal, "Brillouin Microwave Filter with enhanced Skirt Selectivity using a Birefringent Fiber”. IEEE Photonics Technology Letters, vol. 31, no. 6, pp. 431-434, February 2019.

- D. Samaniego, B. Vidal, "Brillouin wavelength-selective all-optical polarization conversion”, Submitted.

\section{Conference publications}

- D. Samaniego, B. Vidal, "Photonic microwave filter based on SBS and balanced detection". In 2017 IEEE Second Ecuador Technical Chapters Meeting (ETCM), pp. 1-6. October 2017.

- A. Zainullin, D. Samaniego, B. Vidal, "Photonic Microwave Filter with Single Passband combining Incoherent and Coherent Techniques". In 2018 IEEE Third Ecuador Technical Chapters Meeting (ETCM), pp. 1-4, October 2018. 


\section{References}

[1] G. P. Agrawal, Nonlinear Fiber Optics, Fifth. Elsevier, 2007.

[2] R. W. Boyd, Nonlinear optics, Third. Elsevier, 2003.

[3] L. Brillouin, "Diffusion de la lumière et des rayons $X$ par un corps transparent homogène. Influence de l'agitation thermique,” Ann. Phys. (Paris)., vol. 9, no. 17, pp. 88-122, 1922.

[4] R. Y. Chiao, C. H. Townes, and B. P. Stoicheff, "Stimulated brillouin scattering and coherent generation of intense hypersonic waves,” Phys. Rev. Lett., vol. 12, no. 21, pp. 592-595, 1964.

[5] E. P. Ippen and R. H. Stolen, "Stimulated Brillouin scattering in optical fibers,” Appl. Phys. Lett., vol. 21, no. 11, p. 1972, 1972.

[6] A. Kobyakov, M. Sauer, and D. Chowdhury, "Stimulated Brillouin scattering in optical fibers,” Appl. Phys. Lett., vol. 2, pp. 1-59, 2010.

[7] R. G. Smith, “Optical Power Handling Capacity of Low Loss Optical Fibers as Determined by Stimulated Raman and Brillouin Scattering,” Appl. Opt., vol. 11, no. 11, pp. 2489-2494, 1972.

[8] D. Williams, X. Bao, and L. Chen, "Effects of polarization on stimulated Brillouin scattering in a birefringent optical fiber,” Photonics Res., vol. 2, no. 5, pp. 126-137, 2014.

[9] R. W. Boyd and K. Rzyzewski, "Noise initiation of stimulated Brillouin acattering," Phys. Rev. A, vol. 42, no. 9, pp. 5514-5521, 1990.

[10] A. Küng, "Laser emission in stimulated Brillouin scattering in optical fibers,” Ecole Polytechnique Féderale de Lausanne, 1997.

[11] S. Le Floch and P. Cambon, "Theoretical evaluation of the Brillouin threshold and the steady-state Brillouin equations in standard single-mode optical fibers," J. Opt. Soc. Am. A, vol. 20, no. 6, p. 1132, 2007.

[12] Stolen R. H., "Polarization Effects in Fiber Raman and Brillouin Lasers,” J. quantum Electron., vol. QE-15, no. 10, 1979. 
[13] T. Horiguchi, N. Shibata, Y. Azuma, and M. Tateda, "Brillouin gain variation due to a polarization-state change of the pump or Stokes fields in standard single-mode fibers,” Opt. Lett., vol. 14, no. 6, p. 329, 2008.

[14] J. N. Damask, Polarization Optics in Telecommunications. Springer Science \& Business Media, 2004.

[15] M. O. Van Deventer and A. J. Boot, "Polarization properties of stimulated Brillouin scattering in single-mode fibers,” J. Light. Technol., vol. 12, no. 4, pp. 585-590, 1994.

[16] E. Brinkmeyer, "Forward-backward transmission in birefringent single-mode fibers: interpretation of polarization-sensitive measurements," Opt. Lett., vol. 6, no. 11, pp. 575-577, 1981.

[17] A. Zadok, E. Zilka, L. Thévenaz, M. Tur, and A. Eyal, "Vector analysis of stimulated Brillouin scattering amplification in standard single-mode fibers," Opt. Express, vol. 16, no. 26, p. 21692, 2008.

[18] L. Chen and X. Bao, “Analytical and numerical solutions for steady state stimulated Brillouin scattering in a single-mode fiber,” Opt. Commun., no. June, pp. 65-70, 1998.

[19] A. M. Scott, K. D. Ridley, and I. Paper, “A Review of Brillouin-Enhanced FourWave Mixing,” IEEE J. Quantum Electron., vol. 25, no. 3, pp. 438-459, 1989.

[20] K. O. Hill, B. S. Kawasaki, and J. D.C., “CW Brillouin laser,” Appl. Phys. Lett., vol. 28, no. 10, pp. 608-609, 1976.

[21] P. Bayvel and I. p. Giles, "Linewidth narrowing in semiconductor laser pumped allfibre brlllouln ring laser,” Electron. Lett., vol. 25, no. 4, pp. 260-262, 1989.

[22] I. S. Grudinin, A. B. Matsko, and L. Maleki, "Brillouin lasing with a CaF2 whispering gallery mode resonator,” Phys. Rev. Lett., vol. 102, no. 4, pp. 1-4, 2009.

[23] S. P. Smith, F. Zarinetchi, and S. Ezekiel, "Narrow-linewidth stimulated Brillouin fiber laser and applications,” Opt. Lett., vol. 16, no. 6, pp. 393-395, 1991.

[24] S. Gundavarapu et al., "Sub-hertz fundamental linewidth photonic integrated Brillouin laser,” Nat. Photonics, vol. 13, no. 1, pp. 60-67, 2019.

[25] N. T. Otterstrom, R. O. Behunin, E. A. Kittlaus, Z. Wang, and P. T. Rakich, “A silicon Brillouin laser,” Appl. Opt., vol. 360, pp. 1113-1116, 2018.

[26] J. Li, M.-G. Suh, and K. Vahala, “Microresonator Brillouin gyroscope,” Optica, vol. 4, no. 3, pp. 346-348, 2017.

[27] T. Horiguchi and M. Tateda, "Optical-fiber-attenuation investigation using stimulated Brillouin scattering between a pulse and a continuous wave,” Opt. Lett., vol. 14, no. 8, pp. 408-410, 1989. 
[28] X. Bao and L. Chen, "Recent progress in Brillouin scattering based fiber sensors," Sensors, vol. 11, pp. 4152-4187, 2011.

[29] X. Bao, J. Dhliwayo, N. Heron, D. J. Webb, and D. A. Jackson, "Experimental and Theoretical Studies on a Distributed Temperature Sensor Based on Brillouin Scattering,” J. Light. Technol., vol. 13, no. 7, pp. 1340-1348, 1995.

[30] J. Urricelqui, A. Zornoza, M. Sagues, and A. Loayssa, "Dynamic BOTDA measurements based on Brillouin phase-shift and RF demodulation," Opt. Express, vol. 20, no. 24, pp. 26942-26949, 2012.

[31] T. Kurashima, T. Horiguchi, H. Izumita, S. I. Furukawa, and Y. Koyamada, "Brillouin optical-fiber time domain reflectometry," IEICE Trans. Commun., vol. 76, no. 4, pp. 382-390, 1993.

[32] H. F. Martins, S. Martin-Lopez, P. Corredera, M. L. Filograno, O. Frazao, and M. Gonzalez-Herraez, "Coherent noise reduction in high visibility phase-sensitive optical time domain reflectometer for distributed sensing of ultrasonic waves," $J$. Light. Technol., vol. 31, no. 23, pp. 3631-3637, 2013.

[33] Y. Mizuno, W. Zou, Z. He, and K. Hotate, "Proposal of Brillouin optical correlationdomain reflectometry (BOCDR),” Opt. Express, vol. 16, no. 16, pp. 12148-12153, 2008.

[34] K. Hotate and T. Hasegawa, "Measurement of Brillouin Gain Spectrum Distribution along an Optical Fiber Using a Correlation-Based Technique - Proposal, Experiment and Simulation,” IEICE Trans. Electron., vol. E83-C, no. 3, pp. 405-412, 2000.

[35] J. Marinelarena, Y. H. Kim, A. Loayssa, and K. Y. Song, "Dual-probe linearly configured BOCDA system with enlarged modulation amplitude," J. Light. Technol., vol. 36, no. 22, pp. 5203-5209, 2018.

[36] K. P. Jackson et al., “Optical Fiber Delay-Line Signal Processing,” IEEE Trans. Microw. Theory Tech., vol. 33, no. 3, pp. 193-210, 1985.

[37] B. Vidal, D. Madrid, J. L. Corral, and J. Marti, "Novel photonic true-time-delay beamformer based on the free-spectral-range periodicity of arrayed waveguide gratings and fiber dispersion,” IEEE Photonics Technol. Lett., vol. 14, no. 11, pp. 1614-1616, 2002.

[38] B. Vidal, J. L. Corral, and J. Marti, "Optical delay line employing an arrayed waveguide grating in fold-back configuration," IEEE Microw. Wirel. Components Lett., vol. 13, no. 6, pp. 238-240, 2003.

[39] J. Palací and B. Vidal, "Tunable optical delay line based on single-sideband suppressed-carrier modulation,” IEEE Photonics Technol. Lett., vol. 25, no. 1, pp. 43-46, 2013.

[40] L. Zhuang, D. Marpaung, M. Burla, W. Beeker, A. Leinse, and C. Roeloffzen, "Low- 
loss, high-index-contrast Si_3N_4/SiO_2 optical waveguides for optical delay lines in microwave photonics signal processing," Opt. Express, vol. 19, no. 23, pp. 23162 23170, 2011.

[41] H. Lee, T. Chen, J. Li, O. Painter, and K. J. Vahala, "Ultra-low-loss optical delay line on a silicon chip," Nat. Commun., vol. 3, p. 867, 2012.

[42] M. Gonzalez-Herraez, K.-Y. Song, and L. Thevenaz, "Optically controlled slow and fast light in optical fibers using stimulated Brillouin scattering,” Appl. Phys. Lett., vol. 87, p. 81113, 2005.

[43] L. Thévenaz, "Slow and fast light in optical fibres," Nat. Photonics, vol. 2, pp. 474 481, 2008.

[44] A. Villafranca, J. A. Lázaro, I. Salinas, and I. Garcés, "Measurement of the linewidth enhancement factor in DFB lasers using a high-resolution optical spectrum analyzer,” IEEE Photonics Technol. Lett., vol. 17, no. 11, pp. 2268-2270, 2005.

[45] M. Sagues and A. Loayssa, "Swept optical single sideband modulation for spectral measurement applications using stimulated Brillouin scattering,” Opt. Express, vol. 18, no. 16, p. 17555, 2010.

[46] “Aragon Photonics.” [Online]. Available: https://aragonphotonics.com/. [Accessed: 08-Apr-2019].

[47] J. Capmany and D. Novak, "Microwave photonics combines two worlds," Nat. Photonics, vol. 1, pp. 319-330, 2007.

[48] B. Vidal, T. Nagatsuma, N. J. Gomes, and T. E. Darcie, "Photonic technologies for millimeter- and submillimeter-wave signals,” Adv. Opt. Technol., vol. 2012, 2012.

[49] D. Marpaung, C. Roeloffzen, R. Heideman, A. Leinse, S. Sales, and J. Capmany, "Integrated microwave photonics," Laser Photonics Rev., vol. 7, no. 4, pp. 506-538, 2013.

[50] D. Marpaung, J. Yao, and J. Capmany, "Integrated microwave photonics," Nat. Photonics, vol. 13, pp. 80-90, 2019.

[51] D. Novak et al., "Radio-over-fiber technologies for emerging wireless systems," IEEE J. Quantum Electron., vol. 52, no. 1, pp. 1-11, 2016.

[52] M. Y. Frankel and R. D. Esman, "True Time-Delay Fiber-Optic Control of an Ultrawideband Array Transmitter/Receiver with Multibeam Capability,” IEEE Trans. Microw. Theory Tech., vol. 43, no. 9, pp. 2387-2394, 1995.

[53] B. Vidal et al., "Simplified WDM optical beamforming network for large antenna arrays,” IEEE Photonics Technol. Lett., vol. 18, no. 10, pp. 1200-1202, 2006.

[54] B. Vidal, M. Á. Piqueras, and J. Martí, "Direction-of-arrival estimation of broadband microwave signals in phased-array antennas using photonic techniques," J. Light. 
Technol., vol. 24, no. 7, pp. 2741-2745, 2006.

[55] A. Meijerink et al., "Novel Ring Resonator-Based Integrated Photonic Beamformer for Broadband Phased Array Receive Antennas - Part I: Design and Performance Analysis,” J. Light. Technol., vol. 28, no. 1, pp. 3-18, 2010.

[56] B. Vidal, T. Mengual, and J. Martí, "Photonic technique for the measurement of frequency and power of multiple microwave signals," IEEE Trans. Microw. Theory Tech., vol. 58, no. 11, pp. 3103-3108, 2010.

[57] B. Vidal, M. A. Piqueras, and J. Martí, "Photonic microwave filter based on spectrum slicing with reconfiguration capability," Electron. Lett. Lett., vol. 41, no. 23, 2005.

[58] J. Palací, G. E. Villanueva, J. V. Galán, J. Martí, and B. Vidal, "Single bandpass photonic microwave filter based on a notch ring resonator," IEEE Photonics Technol. Lett., vol. 22, no. 17, pp. 1276-1278, 2010.

[59] A. Bockelt, J. Palaci Lopez, and B. Vidal, "All-Fiber Centralized Architecture for Parallel Terahertz Sensors," IEEE Trans. Terahertz Sci. Technol., vol. 5, no. 1, pp. 137-144, 2015.

[60] K. J. Williams and R. D. Esman, "Stimulated Brillouin scattering for improvement of microwave fibre-optic link efficiency," Electron. Lett., vol. 30, no. 23, pp. 19651966, 1994.

[61] A. Loayssa, D. Benito, and M. J. Garde, "Optical carrier Brillouin processing of microwave photonic signals.,” Opt. Lett., vol. 25, no. 17, pp. 1234-1236, 2000.

[62] A. Loayssa and F. J. Lahoz, "Broad-band RF photonic phase shifter based on stimulated brillouin scattering and single-sideband modulation," IEEE Photonics Technol. Lett., vol. 18, no. 1, pp. 208-210, 2006.

[63] J. Li, H. Lee, and K. J. Vahala, "Microwave synthesizer using an on-chip Brillouin oscillator," Nat. Commun., vol. 4, pp. 1-7, 2013.

[64] Alan Rogers, Polarization in Optical Fibers. Artech House, 2008.

[65] X. L. Wang et al., "18-Qubit Entanglement with Six Photons' Three Degrees of Freedom,” Phys. Rev. Lett., vol. 120, no. 26, pp. 1-5, 2018.

[66] M. Padgett, J. Leach, E. Botvinick, D. Preece, R. Bowman, and S. Keen, "Independent polarisation control of multiple optical traps," Opt. Express, vol. 16, no. 20, p. 15897, 2008.

[67] R. Ulrich and M. Johnson, "Single-mode fiber-optical polarization rotator," Appl. Opt., vol. 18, no. 11, pp. 1857-1861, 1979.

[68] H. C. Lefevre, "Single-Mode Fibre Fractional Wave Devices and Polarisation," Electron. Lett., vol. 16, no. 20, 1980. 
[69] B. G. Koehler and J. E. Bowers, "In-line single-mode fiber polarization controllers at 1.55, 1.30, and $0.63 \mu \mathrm{m}$," Appl. Opt., vol. 24, no. 3, p. 349, 2009.

[70] D. Bar, "Fiber squeezer polarization controller with low activation loss," 2002.

[71] F. Heismann and M. S. Whalen, "Broadband reset-free automatic polarisation controller,” Electron. Lett., vol. 27, no. 4, p. 377, 2007.

[72] M. Ferrario, L. Marazzi, P. Martelli, M. Cirigliano, and M. Martinelli, "Evidence of Raman-induced polarization pulling,” Opt. Express, vol. 17, no. 2, p. 947, 2009.

[73] B. Vidal, "Photonic millimeter-wave frequency multiplication based on cascaded four-wave mixing and polarization pulling,” Opt. Lett., vol. 37, no. 24, p. 5055, 2012.

[74] Z. Shmilovitch et al., "Dual-pump push-pull polarization control using stimulated Brillouin scattering," Opt. Express, vol. 19, no. 27, p. 25873, 2011.

[75] V. V. Kozlov, M. Barozzi, A. Vannucci, and S. Wabnitz, "Lossless polarization attraction of copropagating beams in telecom fibers,” J. Opt. Soc. Am. B, vol. 30, no. 3, p. 530, 2013.

[76] J. Fatome, S. Pitois, P. Morin, and G. Millot, "Observation of light-by-light polarization control and stabilization in optical fibre for telecommunication applications,” Opt. Express, vol. 18, no. 15, p. 15311, 2010.

[77] S. Pitois, G. Millot, and S. Wabnitz, "Nonlinear polarization dynamics of counterpropagating waves in an isotropic optical fiber: theory and experiments," $J$. Opt. Soc. Am. B, vol. 18, no. 4, p. 432, 2007.

[78] V. V. Kozlov, J. Nuño, and S. Wabnitz, "Theory of lossless polarization attraction in telecommunication fibers: erratum,” J. Opt. Soc. Am. B, vol. 29, no. 1, p. 153, 2011.

[79] J. E. Heebner, R. S. Bennink, R. W. Boyd, and R. A. Fisher, "Conversion of unpolarized light to polarized light with greater than $50 \%$ efficiency by photorefractive two-beam coupling,” Opt. Lett., vol. 25, no. 4, p. 257, 2000.

[80] M. Pagani, D. Marpaung, D.-Y. Choi, S. J. Madden, B. Luther-Davies, and B. J. Eggleton, "Tunable wideband microwave photonic phase shifter using on-chip stimulated Brillouin scattering," Opt. Express, vol. 22, no. 23, p. 28810, 2014.

[81] Y. Ohishi et al., "Fabrication and characterization of a chalcogenide-tellurite composite microstructure fiber with high nonlinearity,” Opt. Express, vol. 17, no. 24, p. 21608, 2009.

[82] B. Morrison et al., "Compact Brillouin devices through hybrid integration on Silicon,” Optica, vol. 4, no. 8, pp. 847-854, 2017.

[83] D. Derickson, Fiber Optica Test and Measurement. Prentice Hall, 1998.

[84] J. Yao, "Photonics to the rescue," IEEE Microw. magzine, no. August, pp. 46-60, 
2015.

[85] B. Vidal, J. L. Corral, and J. Martí, "All-optical WDM multi-tap microwave filter with flat bandpass,” Opt. Express, vol. 14, no. 2, p. 581, 2006.

[86] V. R. Supradeepa et al., "Comb-based radiofrequency photonic filters with rapid tunability and high selectivity," Nat. Photonics, vol. 6, no. 3, pp. 186-194, 2012.

[87] J. Palací et al., "Tunable photonic microwave filter with single bandpass based on a phase-shifted fiber bragg grating," IEEE Photonics Technol. Lett., vol. 22, no. 19, pp. 1467-1469, 2010.

[88] Y. Stern et al., "Tunable sharp and highly selective microwave-photonic band-pass filters based on stimulated Brillouin scattering," Photonics Res., vol. 2, no. 4, p. B18, 2014.

[89] B. Vidal, M. A. Piqueras, and J. Martí, "Tunable and reconfigurable photonic microwave filter based on stimulated Brillouin scattering," Opt. Lett., vol. 32, no. 1, p. 23, 2007.

[90] A. Byrnes et al., "Photonic chip based tunable and reconfigurable narrowband microwave photonic filter using stimulated Brillouin scattering,” Opt. Express, vol. 20, no. 17, p. 18836, 2012.

[91] W. Wei, Y. Jaouën, and W. Hu, "Bandwidth-tunable narrowband rectangular optical filter based on stimulated Brillouin scattering in optical fiber,” Opt. Express, vol. 22, no. 19, pp. 23249-23260, 2014.

[92] M. S. Rasras et al., "Demonstration of a Tunable Microwave-Photonic Notch Filter Using Low-Loss Silicon Ring Resonators,” J. Light. Technol., vol. 27, no. 12, pp. 2105-2110, 2009.

[93] B. Vidal, J. Palací, and J. Capmany, "Reconfigurable photonic microwave filter based on four-wave mixing," IEEE Photonics J., vol. 4, no. 3, pp. 759-764, 2012.

[94] W. Zhang and R. A. Minasian, "Switchable and tunable microwave photonic brillouin-based filter,” IEEE Photonics J., vol. 4, no. 5, pp. 1443-1455, 2012.

[95] W. Wei, L. Yi, Y. Jaouen, M. Morvan, and W. Hu, "Brillouin Rectangular Optical Filter with Improved Selectivity and Noise Performance," IEEE Photonics Technol. Lett., vol. 27, no. 15, pp. 1593-1596, 2015.

[96] D. Marpaung et al., "Low-power, chip-based stimulated Brillouin scattering microwave photonic filter with ultrahigh selectivity," Optica, vol. 2, no. 2, p. 76, 2015.

[97] H. Tang, Y. Yu, C. Zhang, Z. Wang, L. Xu, and X. Zhang, “Analysis of performance optimization for a microwave photonic filter based on stimulated brillouin scattering,” J. Light. Technol., vol. 35, no. 20, pp. 4375-4383, 2017. 
[98] Lilin Yi, Wei Wei, Y. Jaouen, and W. Hu, "Ideal rectangular microwave photonic filter with hihg selectivity based on stimulated Brillouin scattering," in Optical Fiber Communication Conference, 2015, p. Tu3F.5.

[99] P. Li, X. Zou, W. Pan, L. Yan, and S. Pan, “Tunable Photonic Radio-Frequency Filter with a Record High Out-of-Band Rejection,” IEEE Trans. Microw. Theory Tech., vol. 65, no. 11, pp. 4502-4512, 2017.

[100] W. Zhang and R. A. Minasian, "Ultrawide tunable microwave photonic notch filter based on stimulated brillouin scattering,” IEEE Photonics Technol. Lett., vol. 24, no. 14, pp. 1182-1184, 2012.

[101] D. Marpaung, B. Morrison, R. Pant, and B. J. Eggleton, "Frequency agile microwave photonic notch filter with anomalously-high stopband rejection,” Opt. Lett., vol. 38, no. 21, pp. 4300-4303, 2013.

[102] B. Morrison et al., "Tunable microwave photonic notch filter using on-chip stimulated Brillouin scattering,” Opt. Commun., vol. 313, pp. 85-89, 2014.

[103] A. Casas-Bedoya, B. Morrison, M. Pagani, D. Marpaung, and B. J. Eggleton, "Tunable narrowband microwave photonic filter created by stimulated Brillouin scattering from a Silicon nanowire,” Opt. Lett., vol. 40, no. 17, pp. 4154-4157, 2015.

[104] S. Preußler, A. Wiatrek, K. Jamshidi, and T. Schneider, "Brillouin scattering gain bandwidth reduction down to 34MHz,” Opt. Express, vol. 19, no. 9, p. 8565, 2011.

[105] X. Han and J. Yao, "Bandstop-to-bandpass microwave photonic filter using a phaseshifted fiber bragg grating,” J. Light. Technol., vol. 33, no. 24, pp. 5133-5139, 2015.

[106] B. P. Lathi, Linear Systems and Signals. Oxford University, 2005.

[107] Hank Zumbahlen, Linear Circuit Design Handbook. Newnes, 2011.

[108] R. A. M. W. Zhang, "Widely tunable single-passband microwave photonic filter based on stimulated Brillouin scattering," IEEE Photon. Technol. Lett., vol. 23, no. 23, pp. 1775-1777, 2011.

[109] H. G. Jerrard, "Transmission of Light through Birefringent and Optically Active Media: the Poincaré Sphere,” J. Opt. Soc. Am., vol. 44, no. 8, pp. 634-640, 1954.

[110] Z. Ou, J. Li, L. Zhang, Z. Dai, and Y. Liu, “An approximate analytic solution of the steady state Brillouin scattering in single mode optical fiber without neglecting the attenuation coefficient,” Opt. Commun., vol. 282, no. 18, pp. 3812-3816, 2009.

[111] R. C. Jones, “A New Calculus for the Treatment of Optical Systems,” J. Opt. Soc. Am., vol. 31, no. 7, pp. 488-493, 1941. 